Louisiana State University

LSU Digital Commons

$1-1-2014$

\title{
Phylogenetics and diversification of tanagers (Passeriformes: Thraupidae), the largest radiation of Neotropical songbirds
}

\author{
Kevin J. Burns \\ San Diego State University \\ Allison J. Shultz \\ San Diego State University \\ Pascal O. Title \\ San Diego State University \\ Nicholas A. Mason \\ San Diego State University \\ F. Keith Barker \\ University of Minnesota Twin Cities
}

See next page for additional authors

Follow this and additional works at: https://digitalcommons.Isu.edu/biosci_pubs

\section{Recommended Citation}

Burns, K., Shultz, A., Title, P., Mason, N., Barker, F., Klicka, J., Lanyon, S., \& Lovette, I. (2014). Phylogenetics and diversification of tanagers (Passeriformes: Thraupidae), the largest radiation of Neotropical songbirds. Molecular Phylogenetics and Evolution, 75 (1), 41-77. https://doi.org/10.1016/ j.ympev.2014.02.006

This Article is brought to you for free and open access by the Department of Biological Sciences at LSU Digital Commons. It has been accepted for inclusion in Faculty Publications by an authorized administrator of LSU Digital Commons. For more information, please contact ir@lsu.edu. 


\section{Authors}

Kevin J. Burns, Allison J. Shultz, Pascal O. Title, Nicholas A. Mason, F. Keith Barker, John Klicka, Scott M. Lanyon, and Irby J. Lovette 


\title{
Phylogenetics and diversification of tanagers (Passeriformes: Thraupidae), the largest radiation of Neotropical songbirds
}

\author{
Kevin J. Burns ${ }^{\mathrm{a}, *}$, Allison J. Shultz ${ }^{\mathrm{a}, \mathrm{b}}$, Pascal O. Title ${ }^{\mathrm{a}, \mathrm{c}}$, Nicholas A. Mason ${ }^{\mathrm{a}, \mathrm{d}}$, \\ F. Keith Barker ${ }^{\mathrm{e}}$, John Klicka ${ }^{\mathrm{f}}$, Scott M. Lanyon ${ }^{\mathrm{e}}$, Irby J. Lovette ${ }^{\mathrm{d}}$ \\ a Department of Biology, San Diego State University, 5500 Campanile Drive, San Diego, CA 92182, USA \\ ${ }^{\mathrm{b}}$ Department of Organismic and Evolutionary Biology, Harvard University, 26 Oxford Street, Cambridge, MA 02138, USA \\ ${ }^{c}$ Department of Ecology and Evolutionary Biology, University of Michigan, Ann Arbor, MI 48109, USA \\ ${ }^{\mathrm{d}}$ Fuller Evolutionary Biology Program, Cornell Lab of Ornithology, Cornell University, 159 Sapsucker Woods Road, Ithaca, NY 14850, USA

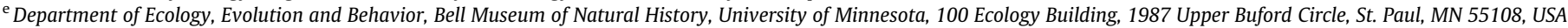 \\ ${ }^{\mathrm{f}}$ Barrick Museum of Natural History, University of Nevada, Las Vegas, NV 89154, USA
}

\section{A R T I C L E I N F O}

\section{Article history:}

Received 1 November 2013

Revised 5 February 2014

Accepted 12 February 2014

Available online 26 February 2014

\section{Keywords:}

Tanager

Thraupidae

Diversification

Darwin's finches

Emberizidae

Sporophila

\begin{abstract}
A B S T R A C T
Thraupidae is the second largest family of birds and represents about $4 \%$ of all avian species and $12 \%$ of the Neotropical avifauna. Species in this family display a wide range of plumage colors and patterns, foraging behaviors, vocalizations, ecotypes, and habitat preferences. The lack of a complete phylogeny for tanagers has hindered the study of this evolutionary diversity. Here, we present a comprehensive, species-level phylogeny for tanagers using six molecular markers. Our analyses identified 13 major clades of tanagers that we designate as subfamilies. In addition, two species are recognized as distinct branches on the tanager tree. Our topologies disagree in many places with previous estimates of relationships within tanagers, and many long-recognized genera are not monophyletic in our analyses. Our trees identify several cases of convergent evolution in plumage ornaments and bill morphology, and two cases of social mimicry. The phylogeny produced by this study provides a robust framework for studying macroevolutionary patterns and character evolution. We use our new phylogeny to study diversification processes, and find that tanagers show a background model of exponentially declining diversification rates. Thus, the evolution of tanagers began with an initial burst of diversification followed by a rate slowdown. In addition to this background model, two later, clade-specific rate shifts are supported, one increase for Darwin's finches and another increase for some species of Sporophila. The rate of diversification within these two groups is exceptional, even when compared to the overall rapid rate of diversification found within tanagers. This study provides the first robust assessment of diversification rates for the Darwin's finches in the context of the larger group within which they evolved.
\end{abstract}

(C) 2014 Elsevier Inc. All rights reserved.

\section{Introduction}

The tanagers (Passeriformes: Thraupidae) represent a major continental radiation, making up an important component of the Neotropical fauna. Species in this clade display a range of plumage colors and patterns, behaviors, morphologies, and ecotypes, and no single physical characteristic defines the group well. This trait diversity displayed among tanagers approaches that seen across the entire radiation of passerine birds, but within tanagers, this evolution has happened on reduced temporal and spatial scales. Because of the extensive character variation seen among tanager

\footnotetext{
* Corresponding author.

E-mail address: kburns@mail.sdsu.edu (K.J. Burns).
}

species, Thraupidae has been long recognized as a problematic clade (e.g., Sclater, 1886; Storer, 1969), with little agreement on its limits. Storer (1970) provided the classic, pre-molecular classification of tanagers that best exemplifies the traditional view; in this classification, the tanagers include 242 mostly colorful, fruiteating birds, and this representation was followed in many subsequent treatments of tanagers (e.g., Burns, 1997; Howard and Moore, 1991; Isler and Isler, 1999). With the advent of molecular data, ideas about what constitutes Thraupidae began to shift. For example, DNA hybridization studies (Bledsoe, 1988; Sibley and Ahlquist, 1990) indicated that many finch-billed New World sparrows (Emberizidae) from the Neotropics belong to the tanager clade. Over two decades of additional genetic work (e.g., Burns, 1997; Burns et al., 2003; Lougheed et al., 2000; Weir et al., 2009; Yuri and Mindell, 2002) have made further progress towards 
defining a monophyletic Thraupidae. However, these studies have mostly explored relationships within different subgroups of tanagers or contained only sparse taxonomic sampling. Recently, Barker et al. (2013) provided the first comprehensive sampling of tanagers and their potential relatives and produced a robust phylogeny that defined a monophyletic Thraupidae. Barker et al. (2013) included six molecular markers and sampled every genus of tanager and every genus of potential tanager relative in the New World, 9-primaried oscine group, representing about $8 \%$ of avian species. These data were subjected to maximum likelihood, Bayesian, and species tree analyses, and all of these analyses consistently defined a monophyletic Thraupidae, with strong support. Combining Barker et al.'s (2013) genus-level sampling with current species-level taxonomies (Clements et al., 2013; Remsen et al., 2013 ) indicates that there are 371 species within Thraupidae. Thus, our newly reconstituted Thraupidae now includes roughly $4 \%$ of all avian species and is the second largest avian family, exceeded only by the New World flycatchers in family Tyrannidae (Clements et al., 2013).

This new view of tanagers indicates the group is even more diverse than previously appreciated. Species now included in Thraupidae were formerly spread across multiple avian families and represent a range of feeding morphologies, plumages, vocal abilities, and habitat preferences. For example, tanagers include such divergent taxa as the Darwin's finches (Burns et al., 2002), nectar-feeding honeycreepers (Burns et al., 2003), multicolored cloud-forest species (Sedano and Burns, 2010), and high altitude (>3000 m) specialists (Campagna et al., 2011). Plumage colors and patterns encompass the full range seen within passerines, including many species with ultraviolet coloration (Burns and Shultz, 2012). With the exception of a few species that occur on islands in the South Atlantic (Ryan et al., 2013), tanagers can now be considered an endemic, Neotropical radiation and represent roughly $12 \%$ of avian species in the region. As now defined, Thraupidae is ecologically diverse and found from northern Mexico through South America, at elevations ranging from coastlines to Andean highlands. Tanagers occur in 20 of the 22 zoogeographic regions and 27 of the 29 terrestrial habitats of the Neotropics (Parker et al., 1996), inhabiting a range of environments from dense tropical rainforest to high altitude grasslands. Nearly every foraging niche is represented in this new Thraupidae, including thickbilled granivores, thin-billed nectar feeders, aerial insect foragers, foliage gleaners, bark probers, and frugivores. Although this diversity has historically caused taxonomic confusion, it is precisely this physical, behavioral, and ecological disparity that makes tanagers an important group to study from an evolutionary perspective.

The first step towards fully understanding this diversity is to develop a robust phylogeny for the group. Although Barker et al. (2013) were able to define a monophyletic clade of tanagers, their sampling included only one representative per genus. Thus, they were unable to explore relationships within tanagers in detail. In this paper, we expand on Barker et al. (2013) and present the most comprehensive phylogenetic tree of tanagers to date, including 353 of the 371 species. Barker et al. (2013) showed that diversification rates of tanagers were $40 \%$ higher than their close relatives and an order of magnitude greater than vertebrates as a whole. Thus, we also use our tree to investigate how diversification has proceeded within this remarkable group.

\section{Materials and methods}

\subsection{Taxon sampling}

We used the genus-level phylogeny of Barker et al. (2013) and the species-level taxonomy of Clements et al. (2013) to guide our taxonomic sampling. There are 371 species in Clements et al. (2013) that belong to genera included within Barker et al.'s Thraupidae, and we included 353 (95\%) of these in our study (Tables 1 and 2). The 18 species we were not able to sample are Conothraupis mesoleuca, Dacnis berlepschi, Embernagra longicauda, Incaspiza laeta, Incaspiza watkinsi, Nemosia rourei, Paroaria nigrogenis, Poospiza lateralis, Sicalis mendozae, Sporophila americana, S. ardesiaca, S. bouvronides, S. murallae, S. nigrorufa, S. bouvreuil, Tangara cabanisi, T. peruviana, and Thraupis glaucocolpa. We also included two individuals that represent subspecific taxa in Clements et al. (2013), Sicalis luteoventris and Poospiza whitii. Clements et al. (2013) treats these as subspecies of S. luteola and P. nigrorufa, respectively. These species were included because, at the time our study was initiated, they were considered full species. Cardinalidae, the cardinal-grosbeaks, is the sister taxon to tanagers (Barker et al., 2013). Thus, we included four representatives of this clade to root our trees: Cardinalis cardinalis, Piranga ludoviciana, Pheucticus tibialis, and Passerina ciris (Tables 1 and 2).

\subsection{Character sampling}

Data for six different molecular markers were used to infer evolutionary relationships among the Thraupidae. We included data from two mtDNA gene regions, cytochrome $b$ (cyt $b$ ) and nicotinamide adenine dehydrogenase subunit 2 (ND2), both of which have been useful in resolving relationships among tanagers (e.g., Sedano and Burns, 2010; Mauck and Burns, 2009). In addition, we sequenced four nuclear loci. These include a protein coding gene, recombination activating gene 1 (RAG1), and three introns: the ninth intron of the sex-linked aconitase 1 (ACO1-I9), myoglobin intron 2 (MB-I2), and $\beta$-fibrinogen intron 5 (FGB-I5). These nuclear makers have been useful in resolving relationships at deeper levels within birds (e.g., Barker et al., 2004, 2013). MtDNA was obtained for all species (Table 1). For nuclear markers, we targeted at least one species per genus and included multiple species when nonmonophyly of that genus was either known or suspected (Table 2). DNA isolation, amplification, and sequencing followed standard protocols (Mauck and Burns, 2009; Sedano and Burns, 2010; Barker et al., 2013). Protein-coding sequences were aligned manually, and intron sequences were aligned using default settings of Clustal X (Larkin, 2007) with slight adjustments made by hand. Tables 1 and 2 report GenBank numbers for all sequences used in this study. Most sequences are either original to this study or from recent studies by ourselves; however, some sequences came from past studies by others (Bellemain et al., 2008; Campagna et al., 2011; Hackett, 1996; Lougheed et al., 2000; Petren et al., 2005; Sato et al., 1999, 2001; Tonnis et al., 2005; Yuri and Mindell, 2002).

\subsection{Phylogenetic analyses}

We inferred phylogenies using both maximum likelihood (ML) and Bayesian approaches. ML methods were implemented using RAxML v7.3.1 (Stamatakis, 2006; Stamatakis et al., 2008) on the XSEDE computing cluster, accessed via the CIPRES Science Gateway v3.1 (Miller et al., 2010). Phylogenies were inferred for each individual gene using ML methods. Each analysis of an intron (ACO1-I9, FGB-I5, or MB-I2) used a single partition, while each coding region (cyt $b, \mathrm{ND} 2$, or RAG1) analysis was partitioned by codon position. RAxML applies a GTR $+\Gamma$ model to each partition, so model testing was not performed for the ML reconstructions. The most likely tree was computed simultaneously with 100 bootstrap replicates for each gene using the rapid bootstrap technique (Stamatakis et al., 2008). Additional analyses were performed with the concatenated dataset containing all genes and taxa. The dataset was partitioned by gene and codon position when applicable for coding regions, resulting in 12 partitions. The most likely tree 
Table 1

Species names, voucher numbers, localities, and GenBank accession numbers of mtDNA sequences for all species included in the study.

\begin{tabular}{|c|c|c|c|c|}
\hline & Voucher/Sample Number $^{\mathrm{a}}$ & Locality & Cyt $b$ & ND2 \\
\hline \multicolumn{5}{|l|}{ Ingroup species } \\
\hline Acanthidops bairdi & LSUMZ B16267 & Costa Rica: San Jose, Cerro de la Muerte, Pan American Highway, km 113 & AF489878 & EU647924 \\
\hline Anisognathus igniventris & FMNH 430092 & Peru: Cuzco, Paucartambo: Pillahuata & EU647961 & EU648006 \\
\hline Anisognathus lacrymosus & IAvH 1755 & Colombia: Caldas, Neira, La Cristalina, Finca La Estrella, Cuenca Alta del Rio Tapias & EU647963 & EU648007 \\
\hline Anisognathus melanogenys & IAvH 478 & Colombia: Magdalena, Santa Marta, San Lorenzo, Sierra Nevada de Santa Marta & EU647965 & EU648009 \\
\hline Anisognathus notabilis & ICN 32717 & Colombia: Narino, Barbacoas & EU647966 & EU648010 \\
\hline Anisognathus somptuosus & LSUMZ B566 & Peru: Puno, Abra de Maruncunca, $10 \mathrm{~km}$ SW San Juan del Oro & AY383090 & EU648011 \\
\hline Bangsia arcaei & USNM B01412 & Panama & EU647968 & EU648013 \\
\hline Bangsia aureocincta & CVA-UV 6463 & Colombia: Valle, El Cairo, Alto Galapagos & EU647969 & EU648014 \\
\hline Bangsia edwardsi & CVA-UV 6461 & Colombia: Narino, 700 msnm ruta hacia Tumaco & EU647971 & - \\
\hline Bangsia edwardsi 2 & CVA-UV 6460 & Colombia: Narino, $700 \mathrm{msnm}$ ruta hacia Tumaco & - & EU648015 \\
\hline Bangsia melanochlamys & ICN 31136 & Colombia: Antioquia, Yarumal, Alto Ventanas, Corcovado & EU647972 & EU648018 \\
\hline Bangsia rothschildi & ANSP 2382 & Ecuador: Esmeraldas, $20 \mathrm{~km}$ NNW of Alto Tambo & EU647973 & EU648017 \\
\hline Buthraupis montana & FMNH 433843 & Peru: Cuzco, Paucartambo, La Esperanza, 39 km (road) NE Paucartambo, 2850 m & EU647978 & EU648022 \\
\hline Buthraupis wetmorei & LSUMZ B337 & Peru: Cajamarca, Cerro Chinguela, 5 km NE Sapalache & EU647980 & EU648027 \\
\hline Calochaetes coccineus & LSUMZ B6134 & Ecuador: Morona Santiago, W slope de Cutucci Yapitya & AY383092 & EU648028 \\
\hline Camarhynchus heliobates & Isabela12 & Ecuador: Galapagos, Isabela & AY700042 & - \\
\hline Camarhynchus pallidus & DBF31 & not provided; see Sato et al. (2001) & AF108792 & - \\
\hline Camarhynchus parvulus & DCB97 & not provided; see Sato et al. (2001) & AF108796 & - \\
\hline Camarhynchus pauper & DB101 & not provided; see Sato et al. (2001) & AF108794 & - \\
\hline Camarhynchus psittacula & DBQ111 & not provided; see Sato et al. (2001) & AF108799 & - \\
\hline Catamblyrhynchus diadema & FMNH 433908 & Peru: Cuzco, Paucartambo, La Esperanza, 39 km (road) NE Paucartambo & JN810051 & - \\
\hline Catamblyrhynchus diadema 2 & LSUMZ B338 & Peru & - & AF447271 \\
\hline Catamenia analis & MACN Or-ct-5118 & Argentina: $8 \mathrm{~km}$ W Villa Ventana, Buenos Aires & JN417908 & - \\
\hline Catamenia analis 2 & ZMUC 116225 & Peru: Apurimac, 7 km S Cotaruse & - & JN810431 \\
\hline Catamenia homochroa & LSUMZ B426 & Peru: Dept. Piura; Cruz Blanca; 33 rd km SW Huancabamba & JN810052 & JN810432 \\
\hline Catamenia inornata & MBM 6465 & Argentina: Tucuman & EF529989 & EF529875 \\
\hline Certhidea fusca & GenovesaM64 & Ecuador: Genovesa, Galapagos & AY672065 & - \\
\hline Certhidea olivacea & Fernandina100 & Ecuador: Fernandina, Galápagos & AY672047 & - \\
\hline Charitospiza eucosma & LSUMZ B15356 & Bolivia: Santa Cruz, Serrania de Huanchaca, 45 km E Florida & JN810053 & JN810433 \\
\hline Chlorochrysa calliparaea & LSUMZ B8103 & Peru: Pasco, Playa Pampo, 8 k NW Cushi on trail to Chaglla & AY383095 & EU648029 \\
\hline Chlorochrysa nitidissima & IAvH 2105 & Colombia: Antioquia, Municipio de Amalfi, Vereda Salasar, Finca Bodega Vieja & EU647981 & EU648030 \\
\hline Chlorochrysa phoenicotis & LSUMZ B34873 & Ecuador: Pichincha, $30 \mathrm{~km}$ Santo Domingo de los Colorados & AY383094 & EU648031 \\
\hline Chlorophanes spiza & LSUMZ B2838 & Peru: Loreto, $1 \mathrm{~km} \mathrm{~N}$ Rio Napo, $157 \mathrm{~km}$ by river NNE Iquitos & AF006215 & JN810434 \\
\hline Chlorornis riefferii & LSUMZ B1859 & Peru: Pasco, Chumbre de Ollon, about 12 km E Oxapampa & AY383093 & EU648032 \\
\hline Chrysothlypis chrysomelas & LSUMZ B2189 & Panama: Darien, about 6 km NW Cana & AF006220 & JN810435 \\
\hline Chrysothlypis salmoni & LSUMZ B11822 & Ecuador: Esmeraldas, El Placer & JN810054 & JN810436 \\
\hline Cissopis leverianus & LSUMZ B1143 & Bolivia: La Paz, Rio Beni, ca. $20 \mathrm{~km}$ by river N Puerto Linares & AY383096 & EU648033 \\
\hline Cnemoscopus rubrirostris & LSUMZ B5624 & Peru: Amazonas, $30 \mathrm{~km}$ by road E Florida on road to Rioja & AF006222 & JN810437 \\
\hline Cnemathraupis aureodorsalis & LSUMZ B3564 & Peru: Huanuco, base of bosque Zapatagocha above NE Acomayo & EU647974 & EU648019 \\
\hline Cnemathraupis eximia & LSUMZ B327 & Peru: Cajamarca, Cerro Chinguela, 5 km NE Sapalache & EU647975 & EU648020 \\
\hline Coereba flaveola & STRI ABCFA2 & Bahamas: Abaco Island & AF382993 & AF383109 \\
\hline Compsospiza baeri & MBM 6457 & Argentina: Tucuman & EF529986 & EF529872 \\
\hline Compsospiza garleppi & LSUMZ B106745 & Bolivia: Cochabamba Department & JN810055 & JN810438 \\
\hline Compsothraupis loricata & LACM 45470 & Brazil: Goias, Ilha do Bananal & JN810056 & - \\
\hline Conirostrum albifrons & LSUMZ B1965 & Peru & AF447365 & AF447273 \\
\hline Conirostrum bicolor & STRI TRCBC1 & Trinidad and Tobago: St. George County & AF383025 & AF383141 \\
\hline Conirostrum cinereum & LSUMZ B8300 & Peru: Pasco, Millpo, E Tambo de Vacas on Pozuzo-Chaglla trail & JN810057 & JN810439 \\
\hline Conirostrum ferrugineiventre & FMNH 391984 & Peru: Cuzco, Urubamba, Pumahuanca & JN810058 & JN810440 \\
\hline Conirostrum leucogenys & LSUMZ B2271 & Panama: Darien, about 6 km NW Cana & JN810059 & JN810441 \\
\hline Conirostrum margaritae & LSUMZ B7293 & Peru: Loreto, Amazonas I. Pasto $80 \mathrm{~km} \mathrm{NE}$ Iquitos $80 \mathrm{~m}$ & EU647892 & EU647925 \\
\hline Conirostrum rufum & FMNH 258531 & Colombia: Cundinamarca, Paramo de Guasca & JN810060 & - \\
\hline Conirostrum sitticolor & ANSP 185901 & Ecuador: Carchi Province & AF383000 & AF383116 \\
\hline
\end{tabular}




\begin{tabular}{|c|c|c|c|c|}
\hline & Voucher/Sample Number ${ }^{\mathrm{a}}$ & Locality & Cyt $b$ & ND2 \\
\hline Conirostrum speciosum & FMNH 334602 & Bolivia: Santa Cruz, Chiquitos, San Jose-San Ignacio Rd, km 69 & AY190168 & JN810442 \\
\hline Conirostrum tamarugense & LSUMZ B103857 & Peru: Arequipa, ca. 20 road km E Chiguata & JN810061 & JN810443 \\
\hline Conothraupis speculigera & LSUMZ B5127 & Peru: Lambayeque, Las Pampas, km 885 Pan-American Hwy, 11 road km from Olmos & AF006223 & JN810444 \\
\hline Coryphaspiza melanotis & LSUMZ B6826 & Bolivia: Beni, 3 k SW San Borja & JN810062 & JN810445 \\
\hline Coryphospingus cucullatus & FMNH 334587 & Bolivia: Santa Cruz, Chiquitos, Purubi, $30 \mathrm{~km} \mathrm{~S} \mathrm{San} \mathrm{Jose} \mathrm{de} \mathrm{Chiquitos}$ & FJ799869 & - \\
\hline Coryphospingus cucullatus 2 & UMMZ 235435 & Captive bird & - & AF447274 \\
\hline Coryphospingus pileatus & FMNH 392719 & Brazil: Sergripe, Caninde do Sao Francisco, Curituba, Fazenda Brejo & FJ799870 & JN810446 \\
\hline Creurgops dentatus & LSUMZ B580 & Peru: Puno, Abra de Maruncunca, $10 \mathrm{~km}$ SW San Juan del Oro & FJ799871 & JN810447 \\
\hline Creurgops verticalis & LSUMZ B7974 & Peru: Pasco, Playa Pampa, 8 km NW Cushi on trail to Chaglla & FJ799872 & JN810448 \\
\hline Cyanerpes caeruleus & LSUMZ B14737 & Bolivia: Santa Cruz, Serriana de Huanchaca, $25 \mathrm{~km} \mathrm{SE}$ Catarata Arco Iris & AF006225 & JN810449 \\
\hline Cyanerpes cyaneus & FMNH 427305 & Brazil: Alagoas & FJ799873 & JN810450 \\
\hline Cyanerpes lucidus & USNM B01322 & Panama & JN810063 & JN810451 \\
\hline Cyanerpes nitidus & FMNH 390048 & Brazil: Rondonia, Cachoeeira Nazare, W bank Rio Jiparana & AY190167 & JN810452 \\
\hline Cyanicterus cyanicterus & USNM B10923 & Guyana & JN810064 & JN810453 \\
\hline Cypsnagra hirundinacea & LSUMZ B15290 & Bolivia: Santa Cruz, Velasco, Pre Parque Nacional Noel Kempff Mercado, $30 \mathrm{~km}$ E Aserradero Moira & AF006226 & JN810454 \\
\hline Dacnis albiventris & LSUMZ B28123 & Peru: Loreto Department & JN810065 & JN810455 \\
\hline Dacnis cayana & LSUMZ B15077 & Bolivia: Santa Cruz, Velasco, 13 km SW Piso Firme & AF006227 & JN810456 \\
\hline Dacnis flaviventer & FMNH 323898 & Peru: Madre de Dios, Hacienda Amazonia & JN810066 & JN810457 \\
\hline Dacnis hartlaubi & AMNH 181785 & Colombia & JN810067 & - \\
\hline Dacnis lineata & FMNH 391223 & Bolivia: El Beni, Hacienda Los Angeles, $10 \mathrm{~km}$ E Riberalta & JN810068 & JN810458 \\
\hline Dacnis nigripes & FMNH 395733 & Brazil: Sao Paulo, Salesopolis, Boraceia Biological Station & JN810069 & - \\
\hline Dacnis venusta & LSUMZ B26588 & Panama: Colon, $17 \mathrm{~km}$ by road NW Gamboa, Rio Agua Salud & FJ799874 & JN810459 \\
\hline Dacnis viguieri & UMMZ 152521 & Colombia: Choco, Rio Jurado & JN810070 & - \\
\hline Diglossa albilatera & AMNH DOT 5023 & Venezuela: Aragua, km 40 on El Junquito/Col. Tovar Road & EU647893 & EU647926 \\
\hline Diglossa baritula & FMNH 393877 & Mexico: Jalisco, Las Joyas, Sierra de Manantlan & EU647894 & EU647927 \\
\hline Diglossa brunneiventris & AMNH DOT 2892 & Bolivia: La Paz Department, Prov. Franz Tamayo, Parque Nacional Apolobamba & EU647896 & EU647928 \\
\hline Diglossa caerulescens & AMNH DOT 5022 & Venezuela: Aragua, $\mathrm{km} 40$ on El Junquito/Col. Tovar road & EU647908 & EU647941 \\
\hline Diglossa carbonaria & LSUMZ B106752 & Bolivia: Cochabamba Department & EU647897 & EU647930 \\
\hline Diglossa cyanea & FMNH 430124 & Peru: Cuzco, Paucartambo, Pillahuata & EU647909 & EU647942 \\
\hline Diglossa duidae & AMNH DOT 9754 & Venezuela: Amazonas, Cerro Yutaje & EU647898 & EU647931 \\
\hline Diglossa glauca & FMNH 430121 & Peru: Cuzco, Paucartambo: San Pedro, $1480 \mathrm{~m}$ & EU647910 & EU647943 \\
\hline Diglossa gloriosa & AMNH 824762 & Venezuela: Merida, Laguna Negra & JN810071 & - \\
\hline Diglossa gloriosissima & IAvH BT7531 & Colombia: Antioquia, Ciudad Bolivar, Farallones, del Citara & EU647900 & EU647932 \\
\hline Diglossa humeralis & USNM B3015 & Ecuador & EU647901 & EU647933 \\
\hline Diglossa indigotica & IAvH BT7532 & Colombia: Antioquia, Yarumal, Alto de Ventanas, Vereda El Rosario-Corcovado, Finca Villa Nueva & EU647911 & EU647944 \\
\hline Diglossa lafresnayii & LSUMZ B351 & Peru: Cajamarca, Cerro Chinguela, 5 km NE Sapalache & AF006229 & EU647934 \\
\hline Diglossa major & FMNH 339722 & Venezuela: Bolivar, Santa Elena Hwy, km 122 & AF290155 & AF290118 \\
\hline Diglossa mystacalis & FMNH 433931 & Peru: Cuzco, Paucartambo, La Esperanza, 39 km (road) NE Paucartambo & EU647903 & EU647936 \\
\hline Diglossa plumbea & AMNH DOT 3646 & Costa Rica: San Jose, Cerro de la Muerte & EU647904 & EU647937 \\
\hline Diglossa sittoides & LSUMZ B22814 & Bolivia: La Paz, B. Saavedra, 83 km by road E Charazani, Cerro Asunta Pata & EU647905 & EU647938 \\
\hline Diglossa venezuelensis & COP 81247 & Venezuela: Anzoategui, Serranía del Turimiquire, Cerro La Launa (El Guamal) & EU647907 & EU647940 \\
\hline Diuca diuca & MBM 6477 & Argentina: Tucuman & EF529984 & EF529870 \\
\hline Diuca speculifera & LSUMZ B22574 & Bolivia: La Paz, Zongo Valley, $7 \mathrm{~km}$ by road $\mathrm{N}$ of summit & JN810072 & JN810460 \\
\hline Dolospingus fringilloides & USNM B11981 & Guyana & JN810073 & JN810461 \\
\hline Donacospiza albifrons & KU 3316 & Paraguay: Misiones, 5 km NW Yabebyry, Estancia Santa Ana & JN810074 & JN810462 \\
\hline Dubusia castaneoventris & LSUMZ B3607 & Peru: Huanuco, Quebrada Shugush, $30 \mathrm{~km}$ on Huanuco-La Union Rd & AY383097 & EU648034 \\
\hline Dubusia taeniata & LSUMZ B7710 & Peru: Huanuco, Unchog Pass NNW Acomayo $3450 \mathrm{~m}$ & AY383098 & EU648035 \\
\hline Emberizoides duidae & USNM 605984 & Venezuela: Amazonas, Mount Duida, elevation & JN810075 & - \\
\hline Emberizoides herbicola & MBM 3721 & Argentina: Corrientes & EF529974 & EF529860 \\
\hline Emberizoides ypiranganus & UWBM 70773 & Argentina: Provincia de Corrientes, Corrientes, 55 km S, 5 km E, along Route 103 & JN810076 & JN810463 \\
\hline Embernagra platensis & FMNH 396034 & Bolivia & EU647912 & EU647945 \\
\hline Eucometis penicillata & LSUMZ B6551 & Bolivia: Santa Cruz, Rio Quizer & FJ799875 & JN810464 \\
\hline Euneornis campestris & FMNH 331119 & Jamaica: Portland, Hollywell Park & AF489885 & EU648036 \\
\hline
\end{tabular}




\begin{tabular}{|c|c|c|c|c|}
\hline & Voucher/Sample Number $^{\mathrm{a}}$ & Locality & Cyt $b$ & ND2 \\
\hline Geospiza conirostris & DBF81 & not provided; see Sato et al. (2001) & AF108769 & - \\
\hline Geospiza difficilis & D12 & not provided; see Sato et al. (2001) & AF108787 & - \\
\hline Geospiza fortis & DCB56 & not provided; see Sato et al. (2001) & AF108772 & - \\
\hline Geospiza fortis 2 & UMMZ 224890 & Ecuador: Galápagos Islands & - & AF447282 \\
\hline Geospiza fuliginosa & DBQ61 & not provided; see Sato et al. (2001) & AF108786 & - \\
\hline Geospiza magnirostris & DBQ11 & not provided; see Sato et al. (2001) & AF108777 & - \\
\hline Geospiza scandens & DB26 & not provided; see Sato et al. (2001) & AF108779 & - \\
\hline Gubernatrix cristata & LSUMZ B51254 & Argentina: Corrientes & JN810077 & - \\
\hline Gubernatrix cristata 2 & MACN 68379 & Argentina: Prov. Buenos Aires; Salinera Universal, Salina de Piedra, Cardenal Cagliero, Partido de Patagones & - & JN810465 \\
\hline Haplospiza rustica & FMNH 433797 & Peru: Cuzco, Paucartambo, La Esperanza, 39 km (road) NE Paucartambo, 2900 m & EU647913 & EU647946 \\
\hline Haplospiza unicolor & FMNH 5186 & Brazil: Sao Paulo & AF290156 & AF290119 \\
\hline Hemispingus atropileus & LSUMZ B1889 & Peru: Pasco, Chumbre de Ollon, about 12 km E Oxapampa & AF006234 & AF383135 \\
\hline Hemispingus calophrys & LSUMZ B547 & Peru: Puno, Valcon, 5 km NNW Quiaca & JN810078 & JN810466 \\
\hline Hemispingus frontalis & LSUMZ B1766 & Peru: Pasco Department & AF383020 & AF383136 \\
\hline Hemispingus goeringi & USNM 263922 & Venezuela: Culata & JN810079 & - \\
\hline Hemispingus melanotis & FMNH 430079 & Peru: Cuzco, Paucartambo: San Pedro & EU647914 & EU647947 \\
\hline Hemispingus parodii & FMNH 316432 & Peru: Cuzco, Abra Malaga, below, on road to Quillabamba & JN810080 & - \\
\hline Hemispingus reyi & ANSP 162616 & Venezuela: Paramo Zumbador & JN810081 & - \\
\hline Hemispingus rufosuperciliaris & LSUMZ B3566 & Peru: Huanuco, base of bosque Zapatagocha above NE Acomayo & JN810082 & JN810467 \\
\hline Hemispingus superciliaris & FMNH 433858 & Peru: Cuzco, Paucartambo, La Esperanza, 39 km (road) NE Paucartambo & JN810083 & JN810468 \\
\hline Hemispingus trifasciatus & FMNH 398459 & Peru: Cuzco, Paucartambo, Puesto de Vigilancia Acjanaco & JN810084 & - \\
\hline Hemispingus verticalis & LSUMZ B320 & Peru: Cajamarca, Cerro Chingueal, 5 km NE Sapalache & JN810085 & JN810469 \\
\hline $\begin{array}{l}\text { Hemispingus } \\
\quad \text { xanthophthalmus }\end{array}$ & LSUMZ B8223 & Peru: Pasco, Millpo, E Tambo de Vacas on Pozuzo-Chaglla trail & JN810086 & JN810470 \\
\hline Hemithraupis flavicollis & LSUMZ B5102 & Peru: Loreto, S Rio Amazonas, ca. 10 km SSW mouth Rio Napo on E bank Quebrada Vainilla & AF006235 & EU647948 \\
\hline Hemithraupis guira & FMNH 427239 & Brazil: Alagoas, Ibateouara, Envenho Ceimba, Usina Serra Grande & JN810087 & JN810471 \\
\hline Hemithraupis ruficapilla & FMNH 395477 & Brazil: Sao Paulo, Boracia & JN810088 & - \\
\hline Heterospingus rubrifrons & LSUMZ B28691 & Panama: Colon, Achitoe road at Rio Providencia & JN810089 & JN810472 \\
\hline Heterospingus xanthopygius & LSUMZ B2324 & Panama: Darien, Cana on E slope Cerro Pirre & EU647915 & EU647949 \\
\hline Idiopsar brachyurus & LSUMZ B22571 & Bolivia: La Paz, Zongo Valley, $7 \mathrm{~km}$ by road $\mathrm{N}$ of summit & EU647916 & EU647950 \\
\hline Incaspiza ortizi & LSUMZ B10382 & Peru: Cajamarca, above Limon Pampa between Calenda and Balsan & JN810090 & JN810473 \\
\hline Incaspiza personata & FMNH 299930 & Peru: Ancash, Huaylas, Quebrada Rurinura & JN810091 & - \\
\hline Incaspiza pulchra & LSUMZ B10387 & Peru: Ancash, just out of Huaylash toward Sucre & JN810092 & JN810474 \\
\hline Iridophanes pulcherrimus & MVZ 169712 & Peru: Dept. Cajamarca, 1 mi N San Jose de Lourdes, Cordillera del Condor & AY190169 & JN810475 \\
\hline Iridosornis analis & LSUMZ B1706 & Peru: Pasco, Santa Cruz, about 9 km SSE Oxapampa & AY383099 & EU648037 \\
\hline Iridosornis jelskii & FMNH 430099 & Peru: Cuzco, Paucartambo: Pillahuata & JN810093 & EU648038 \\
\hline Iridosornis porphyrocephalus & CVA-UV 6454 & Colombia: Alto Ventanas, Yarumal, Antioquia & EU647983 & EU648039 \\
\hline Iridosornis reinhardti & LSUMZ B3538 & Peru: Huanuco, Unchog, pass between Churrrubamba and Hola Paty, NNW Acomayo & EU647985 & EU648041 \\
\hline Iridosornis rufivertex & LSUMZ B371 & Peru: Dept. Cajamarca; Cerro Chinguela, 5 km NE Sapalache & JN810094 & JN810476 \\
\hline Lanio aurantius & MBM 8738 & Honduras: Depto. Atlantida, La Ceiba, 9.7 km SW Rio Quebrada & FJ799877 & JN810477 \\
\hline Lanio fulvus & LSUMZ B2694 & Peru: Loreto, $1 \mathrm{~km} \mathrm{~N}$ Rio Napo, $157 \mathrm{~km}$ by river NNE Iquitos & EU647917 & EU647951 \\
\hline Lanio leucothorax & STRI JTW572 & Panama: Cocle, El Cope National Park & FJ799879 & JN810478 \\
\hline Lanio versicolor & LSUMZ B1014 & Bolivia: La Paz, Rio Beni, ca. $20 \mathrm{~km}$ by river N Puerto Linares & FJ799878 & JN810479 \\
\hline Lophospingus griseocristatus & FMNH 334558 & Bolivia: Cochabamba, Cochabamba-Oruro Rd, km 29 & EU647987 & EU648043 \\
\hline Lophospingus pusillus & MBM 6491 & Argentina: Tucuman & EF529992 & EF529878 \\
\hline Loxigilla barbadensis & STRI BA-LNO17 & Barbados: Apes Hill & HQ153056 & HQ153075 \\
\hline Loxigilla noctis & STRI DO-LNO3 & Dominica: Springfield & HQ153057 & HQ153076 \\
\hline Loxigilla portoricensis & LSUMZ B11351 & Puerto Rico: Cabo Rojo, Boqueron, Penones de Melones, 1 km WNW intersection routes 301 and 303 & AF489886 & EU648044 \\
\hline Loxigilla violacea & AMNH 25433 & Dominican Republic: Independencia & AF489887 & HQ153077 \\
\hline Loxipasser anoxanthus & FMNH 331107 & Jamaica: Surrey, Portland, Hollywell Park & AF489888 & EU648045 \\
\hline Melanodera melanodera & FMNH 120780 & Argentina: Tierra del Fuego, San Sebastian & JN810095 & - \\
\hline Melanodera xanthogramma & AMNH DOT 12115 & Argentina: Departamento Bariloche, Rio Negro & EU647918 & EU647952 \\
\hline
\end{tabular}




\begin{tabular}{|c|c|c|c|c|}
\hline & Voucher/Sample Number ${ }^{\mathrm{a}}$ & Locality & Cyt $b$ & ND2 \\
\hline Melanospiza richardsoni & Meri-CMB95 & Saint Lucia & AF310043 & - \\
\hline Melanospiza richardsoni 2 & SL-MRI2 & Saint Lucia & - & EF567909 \\
\hline Melopyrrha nigra & $\begin{array}{l}\text { Not provided, see Lougheed et al. } \\
(2000)\end{array}$ & Cuba: Cayo Coco & AY005219 & - \\
\hline Melopyrrha nigra 2 & FMNH 342954 & captive bird & - & EU648046 \\
\hline Nemosia pileata & LSUMZ B7295 & Peru: Loreto, Amazonas I. Pasto 80 km NE Iquitos 80 m & AF006241 & JN810480 \\
\hline Neothraupis fasciata & LSUMZ B13914 & Bolivia: Santa Cruz, Serrania de Huanchaca, 45 km E Florida & AY383100 & EU648047 \\
\hline Nephelornis oneilli & LSUMZ B8402 & Peru: Pasco, Millpo, E Tambo de vacas on Pozuzo-Chaglla trail & AF006243 & JN810481 \\
\hline Nesospiza acunhae & ITU35 & Inaccessible Island, Tristan da Cunha & JN810096 & JN810482 \\
\hline Nesospiza wilkinsi & NW5 & Nightingale Island, Tristan da Cunha & DQ886520 & - \\
\hline Nesospiza wilkinsi 2 & NW17 & Nightingale Island, Tristan da Cunha & - & JN810483 \\
\hline Orchesticus abeillei & FMNH 267940 & Brazil: Sao Paulo, Boa Vista, Rio Ipiranga & JN810097 & - \\
\hline Oreomanes fraseri & LSUMZ B2069 & Peru: Lima, ca. 13 road km W Milloc & AF006244 & EU647953 \\
\hline Oryzoborus angolensis & Oran-CMB241 & Ecuador: Santo Domingo & AF310055 & - \\
\hline Oryzoborus angolensis 2 & FMNH 433798 & Peru: Madre de Dios, Moskitania, 13.4 km NNW Atalaya, I bank Alto Madre de Dios & - & JN810484 \\
\hline Oryzoborus atrirostris & ZMUC 123039 & Ecuador: Pastaza, N Canelos & JN810098 & JN810485 \\
\hline Oryzoborus crassirostris & FMNH 339668 & Venezuela: Sucre, Guraunos, 14 km SSE & AF489890 & JN810486 \\
\hline Oryzoborus funereus & MBM 8980 & Honduras: Atlantida & EF529963 & EF529851 \\
\hline Oryzoborus maximiliani & LSUMZ B11908 & Ecuador: Esmeraldas, El Placer & EU647919 & EU647954 \\
\hline Oryzoborus nuttingi & AMNH 787330 & Costa Rica: Guanacaste, Laguna Arenal, Tronadora & JN810099 & - \\
\hline Parkerthraustes humeralis & LSUMZ B9328 & Boliva: Pando & EF530024 & EF529917 \\
\hline Paroaria baeri & FMNH 356584 & Brazil: Mato Grosso; Sao Domingos, Rio das Mortes & JN810100 & - \\
\hline Paroaria capitata & UWBM jag1837 & Argentina: Corrientes & EF529977 & EF529863 \\
\hline Paroaria coronata & FMNH 394390 & Bolivia & EU647990 & EU648049 \\
\hline Paroaria dominicana & FMNH 392736 & Brazil: Sergripe, Caninde do Sao Francisco, Curituba, Fazenda Porto Belo & EF529994 & EF529880 \\
\hline Paroaria gularis & FMNH 323625 & Peru: Madre de Dios, Hacienda Amazonia & EU647989 & EU648050 \\
\hline Phrygilus alaudinus & MBM 6470 & Argentina: Tucuman & EF529981 & EF529867 \\
\hline Phrygilus atriceps & MBM 5307 & Argentina: Jujuy & EF529982 & EF529868 \\
\hline Phrygilus carbonarius & AMNH DOT 10373 & Argentina: Neuquen, Departamento Anelo, Sierra Auca Mahuida & JN810101 & JN810487 \\
\hline Phrygilus dorsalis & MBM 6476 & Argentina: Tucuman & EF529983 & EF529869 \\
\hline Phrygilus erythronotus & LSUMZ B103892 & Peru: Tacna, Tacna-Llave Rd, ca. 57 km NE Tarata & JN810102 & JN810488 \\
\hline Phrygilus fruticeti & MBM 5412 & Argentina: Jujuy, Tilcara, $18 \mathrm{~km} \mathrm{~S}$ & JN810103 & JN810489 \\
\hline Phrygilus gayi & MBM 6475 & Argentina: Tucuman, Amaicha del Valle $12 \mathrm{~km} \mathrm{~S}, 12 \mathrm{~km} \mathrm{E}$ & JN810104 & JN810490 \\
\hline Phrygilus patagonicus & MACN 42511 & Argentina: El Bolson, Rio Negro & JN810105 & JN810491 \\
\hline Phrygilus plebejus & MBM 5310 & Argentina: Jujuy & EF529979 & EF529865 \\
\hline Phrygilus punensis & LSUMZ B61455 & Peru: Apurimac, SE Abancay, Lloccahuani & JN417901 & - \\
\hline Phrygilus unicolor & MBM 6471 & Argentina: Tucuman & EF529980 & EF529866 \\
\hline Piezorina cinerea & LSUMZ B5169 & Peru: Lambayeque, Las Pampas, km 885 Pan-American Hwy, 11 road km from Olmos & JN810106 & JN810492 \\
\hline Pinaroloxias inornata & DBT131 & Not provided; see Sato et al. (2001) & AF108790 & - \\
\hline Pipraeidea bonariensis & FMNH 433891 & Peru: Cuzco, Paucartambo, La Esperanza, 39 km (road) NE Paucartambo & EU647997 & EU648100 \\
\hline Pipraeidea melanonota & LSUMZ B12070 & Ecuador: Pichincha, Mindo & AY383101 & EU648051 \\
\hline Platyspiza crassirostris & DBT72 & Not provided; see Sato et al. (2001) & AF108802 & - \\
\hline Poospiza alticola & ZMUC 0665 & Peru: Ancash & AY005198 & - \\
\hline Poospiza alticola 2 & ZMUC 116453 & Peru: Ancash, C. Blanc, Andavite, Rurichinc. & - & JN810493 \\
\hline Poospiza boliviana & LSUMZ B1198 & Bolivia: La Paz Department & AY005201 & JN810494 \\
\hline Poospiza cabanisi & CUMV 50679 & Uruguay: Artigas, Arroyo Mandiyu & EU647920 & EU647955 \\
\hline Poospiza caesar & ZMUC 0667 & Peru: Andamarca, Ayacucho & AY005202 & - \\
\hline Poospiza cinerea & USNM B05912 & Argentina & FJ799880 & JN810495 \\
\hline Poospiza erythrophrys & MBM 5491 & Argentina: Salta & EF529987 & EF529873 \\
\hline Poospiza hispaniolensis & LSUMZ B5205 & Peru & AY005205 & - \\
\hline Poospiza hispaniolensis 2 & LSUMZ B24977 & captive, Louisiana, Baton Rouge, C. Gordon Barney Aviary, captive & - & JN810496 \\
\hline Poospiza hypochondria & MBM 5302 & Argentina: Salta & EF529985 & EF529871 \\
\hline
\end{tabular}




\begin{tabular}{|c|c|c|c|c|}
\hline & Voucher/Sample Number $^{a}$ & Locality & Cyt $b$ & ND2 \\
\hline Poospiza melanoleuca & $\begin{array}{l}\text { Not provided, see Lougheed et al. } \\
(2000)\end{array}$ & Argentina: Quimili, Santiago del Estero & AY005208 & - \\
\hline Poospiza melanoleuca 2 & MBM 5316 & Argentina: Salta, J.V. Gonzalez, 14 km NE & - & JN810497 \\
\hline Poospiza nigrorufa & AMNH DOT 9907 & Argentina: Buenos Aires, Partido Magdallena & JN810107 & JN810498 \\
\hline Poospiza ornata & $\begin{array}{l}\text { Not provided, see Lougheed et al. } \\
(2000)\end{array}$ & Argentina: Amanao, Catamarca & AY005213 & - \\
\hline Poospiza ornata 2 & AMNH DOT 9515 & locality unknown & - & JN810499 \\
\hline Poospiza rubecula & FMNH 299931 & Peru: Ancash, Quitacocha, Huaylas & JN810108 & - \\
\hline Poospiza thoracica & LACM 29015 & Brazil: Rio de Janeiro, Itatiaia & JN810109 & - \\
\hline Poospiza torquata & KU 2838 & Paraguay: Presidente Hayes, Campo Largo, $5 \mathrm{~km} \mathrm{~S}$ & JN810110 & - \\
\hline Poospiza torquata 2 & MBM 6455 & Argentina: Tucuman & - & EF529877 \\
\hline Poospiza whitii & LSUMZ B6573 & Bolivia: Santa Cruz, 2.5 km N Tambo & JN810111 & JN810500 \\
\hline Porphyrospiza caerulescens & LSUMZ B13860 & Bolivia: Santa Cruz & EF529978 & EF529864 \\
\hline Pyrrhocoma ruficeps & MVZ 165617 & Paraguay: Dept. Itapu, El Tirol, $19.5 \mathrm{~km}$ by road NNE Encarnacion & AF006249 & - \\
\hline Pyrrhocoma ruficeps 2 & KU 91447 & Paraguay: Itapua, San Rafael National Park; Parabel & - & JN810501 \\
\hline Ramphocelus bresilius & Not provided, see Hackett (1996) & captive birds & U15724 & - \\
\hline Ramphocelus carbo & LSUMZ B4988 & Peru: Loreto; S Río Amazonas, 10 km SSW Río Napo on E bank Quebrada Vainilla & U15723 & - \\
\hline Ramphocelus carbo 2 & FMNH 430084 & Peru: Cuzco, Paucartambo: San Pedro & - & JN810502 \\
\hline Ramphocelus costaricensis & LSUMZ B16144 & Costa Rica: Prov. Puntarenas; 2 km SE Dominical & U15720 & U15711 \\
\hline Ramphocelus dimidiatus & LSUMZ B16559 & Panama: Panama Province, Old Gamboa Road-golf course, 4 km NW of Paraiso & FJ799881 & JN810503 \\
\hline Ramphocelus flammigerus & USNM B01238 & Panama: Punta Alegre, Peninsula Veliente & FJ799882 & - \\
\hline Ramphocelus flammigerus 2 & USNM 607943 & Panama: Bocas Del Toro & - & JN810504 \\
\hline Ramphocelus melanogaster & LSUMZ B44693 & Peru: Dept. San Martin; 33 km NE Florida & FJ799883 & JN810505 \\
\hline Ramphocelus nigrogularis & LSUMZ B2850 & Peru: Dpto. Loreto; $1 \mathrm{~km}$ N Río Napo, $157 \mathrm{~km}$ by river NNE Iquitos & U15721 & - \\
\hline Ramphocelus nigrogularis 2 & FMNH 323781 & Peru: Madre de Dios, Hacienda Amazonia & - & JN810506 \\
\hline Ramphocelus passerinii & MBM 8627 & Honduras: Atlantida & EF529965 & EF529853 \\
\hline Ramphocelus sanguinolentus & FMNH 343376 & Mexico: Veracruz, El Bastonal, 3 km S, 3 km E, Sierra de Santa Martha & U15718 & JN810507 \\
\hline Rhodospingus cruentus & LSUMZ B5184 & Peru: Lambayeque, Las Pampas, km 885 Pan-American Hwy, 11 road km from Olmos & FJ799884 & JN810508 \\
\hline Rowettia goughensis & GB18 & Gough Island & JN810112 & JN810509 \\
\hline Saltator albicollis & STRI cCSAL1 & Trinidad and Tobago: Chacachacare Island & AF383107 & AF281023 \\
\hline Saltator atriceps & FMNH 343357 & Mexico: Veracruz & EF530019 & EF529912 \\
\hline Saltator atricollis & LSUMZ B15381 & Bolivia: Santa Cruz & EF530021 & EF529914 \\
\hline Saltator atripennis & ANSP 3491 & Ecuador: Azuay & JN810113 & JN810510 \\
\hline Saltator aurantiirostris & UWBM 54506 & Argentina: Tucuman & EF530017 & EF529910 \\
\hline Saltator cinctus & LSUMZ B6233 & Ecuador: Prov. Morona-Santiago & JN810114 & JN810511 \\
\hline Saltator coerulescens & UWBM gav817 & Argentina: Corrientes & EF530013 & EF529906 \\
\hline Saltator fuliginosus & MACN P1674 & Brazil: Sao Paulo Fazenda Barreiro Rico & JN810115 & JN810512 \\
\hline Saltator grossus & LSUMZ B16063 & Costa Rica: Herredia & EF530018 & EF529911 \\
\hline Saltator maxillosus & FMNH 311035 & Brazil: Rio de Janeiro; Parque Nacional do Itatiaia & JN810116 & - \\
\hline Saltator maximus & FMNH 433810 & Peru: Cuzco, Paucartambo, Consuelo, 15.9 km SW Pilcopata & JN810117 & - \\
\hline Saltator maximus 2 & ZMUC 123036 & Ecuador & - & JN810513 \\
\hline Saltator nigriceps & LSUMZ B183 & Peru: Piura & EF530016 & EF529909 \\
\hline Saltator orenocensis & COP FL2-04//2259 & Venezuela: Falcon, Carretara Coro-Curimagua & JN810118 & JN810514 \\
\hline Saltator rufiventris & LSUMZ B106750 & Bolivia: Cochabamba & EF530025 & EF529918 \\
\hline Saltator similis & UWBM 70491 & Argentina: Provincia de Corrientes, Corrientes, Manuel Derqui & JN810119 & JN810515 \\
\hline Saltator striatipectus & LSUMZ B449 & Peru: Lambayeque & JN810120 & JN810516 \\
\hline Saltatricula multicolor & MVZ 179401 & captive bird & AF489892 & - \\
\hline Saltatricula multicolor 2 & MBM 5447 & Argentina: Salta & - & EF529913 \\
\hline Schistochlamys melanopis & LSUMZ B9669 & Bolivia: Pando, Nicholas Suarez, $12 \mathrm{~km}$ by road $\mathrm{S}$ of Cojiba, $8 \mathrm{~km} \mathrm{~W}$ on road to Mucden & AY383102 & EU648052 \\
\hline Schistochlamys ruficapillus & FMNH 344774 & Brazil: Goias, Goiania & EU647991 & - \\
\hline Sericossypha albocristata & LSUMZ B5630 & Peru: Amazonas, $30 \mathrm{~km}$ by road E Florida on road to Rioja & AF006251 & JN810517 \\
\hline Sicalis auriventris & MACN 35187 & Argentina: Los Molles, Mendoza & JN810121 & - \\
\hline Sicalis citrina & LSUMZ B15400 & Bolivia: Santa Cruz, Serrania De Huanchaca, 45 km E Florida & JN810122 & JN810518 \\
\hline
\end{tabular}

(continued on next page) 


\begin{tabular}{|c|c|c|c|c|}
\hline & Voucher/Sample Number $^{\mathrm{a}}$ & Locality & Cyt $b$ & ND2 \\
\hline Sicalis columbiana & FMNH 391601 & Brazil: Amapa, Tartatugalzinho, Fazenda Sao Bento & JN810123 & JN810519 \\
\hline Sicalis flaveola & UWBM 70184 & Argentina: Provincia de Corrientes, Corrientes, Manuel Derqui & JN810124 & JN810520 \\
\hline Sicalis lebruni & MACN 52328 & Argentina: Chubut, Punta Tombo & JN810125 & - \\
\hline Sicalis lutea & FMNH 391932 & Peru: Ayacucho, Ocana & EU647921 & EU647956 \\
\hline Sicalis luteiventris & MACN 68470 & Argentina: Prov. Buenos Aires; Lago Parque La Salada, Pedro Luro & JN810126 & JN810521 \\
\hline Sicalis luteocephala & ZMUC 116483 & Bolivia: Cochabamba, Cerro Kehuinal & JN810127 & JN810522 \\
\hline Sicalis luteola & FMNH 389274 & Brazil: Roraima, Fazenda Santa Cecilia, E Bank Rio Branco, across from boa Vista & AF489893 & EU647957 \\
\hline Sicalis olivascens & MBM 5435 & Argentina: Jujuy & EF529988 & EF529874 \\
\hline Sicalis raimondii & FMNH 287878 & Peru: Ayacucho, Ocana & JN810128 & - \\
\hline Sicalis taczanowskii & LSUMZ B5244 & Peru: Lambayeque, Las Pampas, km 885 Pan-American Hwy, 11 road km from Olmos & JN810129 & JN810523 \\
\hline Sicalis uropygialis & ZMUC 116495 & Peru: Junin, $3 \mathrm{~km} \mathrm{~S}$ of Ondores & - & JN810524 \\
\hline Sporophila albogularis & FMNH 392743 & Brazil: Alagoas, Piranhas, Fazenda Bela Vista & JN810130 & JN810525 \\
\hline Sporophila caerulescens & FMNH 334570 & Bolivia: Santa Cruz, Chiquitos, Purubi, 30 km S San Jose de Chiquitos & JN810132 & JN810527 \\
\hline Sporophila castaneiventris & FMNH 433815 & Peru: Madre de Dios, Moskitania, 13.4 km NNW Atalaya, I bank Alto Madre de Dios, $480 \mathrm{~m}$ & JN810133 & JN810528 \\
\hline Sporophila cinnamomea & MACN 52373 & Argentina: Prov. Entre Rios; Arroyo Barú & JN810134 & - \\
\hline Sporophila collaris & FMNH 334564 & Bolivia: El Beni, Laguna Suarez, 5 km SW Trinidad & JN810135 & JN810529 \\
\hline Sporophila corvina & STRI GMS2200 & Panama: Chiriqui, $\mathrm{N}$ of Bahia de Charco Azul, $3 \mathrm{~km} \mathrm{~W}$ of Divala & JN810136 & JN810530 \\
\hline Sporophila falcirostris & MACN 39080 & Argentina: Prov. Misiones; Arroyo Urugua-i, km. 40 & JN810137 & - \\
\hline Sporophila frontalis & LACM 27909 & Brazil: Rio de Janiero, Terezopolis & JN810138 & - \\
\hline Sporophila hypochroma & LSUMZ B15265 & Bolivia: Santa Cruz, Velasco, Pre Parque Nacional Noel Kempff Mercado, 30 km E Aserradero Moira & JN810139 & JN810531 \\
\hline Sporophila hypoxantha & FMNH 334574 & Bolivia: Santa Cruz, Chiquitos, San Jose-San Ignacio Rd, km 69 & JN810140 & JN810532 \\
\hline Sporophila intermedia & FMNH 389269 & Brazil: Roraima, Fazenda Santa Cecilia, E Bank Rio Branco, across from boa Vista & EU647922 & EU647958 \\
\hline Sporophila leucoptera & FMNH 334573 & Bolivia: El Beni, Laguna Suarez, 5 km SW Trinidad & JN810141 & JN810533 \\
\hline Sporophila lineola & FMNH 390057 & Brazil: Rondonia, Cachoeeira Nazare, W bank Rio jiparana & JN810142 & JN810534 \\
\hline Sporophila luctuosa & FMNH 433818 & Peru: Madre de Dios, Moskitania, 13.4 km NNW Atalaya, I bank Alto Madre de Dios, $480 \mathrm{~m}$ & JN810143 & JN810535 \\
\hline Sporophila melanogaster & AMNH 315888 & Brazil: Paccaria, Rio Grande do Sul & JN810144 & - \\
\hline Sporophila minuta & FMNH 389270 & Brazil: Roraima, Fazenda Santa Cecilia, E Bank Rio Branco, across from boa Vista & JN810145 & JN810536 \\
\hline Sporophila nigricollis & Spni-CB191 & Ecuador: Santo Domingo & AF310053 & - \\
\hline Sporophila nigricollis 2 & FMNH 427217 & Brazil: Alagoas, Ibateouara, Envenho Ceimba, Usina Serra Grande & - & JN810537 \\
\hline Sporophila palustris & KU 3689 & Paraguay: Itapua, San Rafael National Park, San Pedro Mi, & JN810146 & JN810538 \\
\hline Sporophila peruviana & LSUMZ B5243 & Peru: Lambayeque, Las Pampas, km 885 Pan-American Hwy, 11 road km from Olmos & JN810147 & JN810539 \\
\hline Sporophila pileata & UWBM 70800 & Argentina: Provincia de Missiones, Posadas, 25 km E, Estancia San Juan & JN810131 & JN810526 \\
\hline Sporophila plumbea & FMNH 389271 & Brazil: Roraima, Fazenda Santa Cecilia, E Bank Rio Branco, across from boa Vista & JN810148 & JN810540 \\
\hline Sporophila ruficollis & FMNH 334582 & Bolivia: Santa Cruz, Chiquitos, Purubi, 30 km s San Jose de Chiquitos & AF489896 & - \\
\hline Sporophila ruficollis 2 & FMNH 334583 & Bolivia: Santa Cruz, Chiquitos, Purubi, $30 \mathrm{~km} \mathrm{~s}$ San Jose de Chiquitos & - & JN810541 \\
\hline Sporophila schistacea & LSUMZ B22584 & Bolivia: La Paz Dept., Prov. B. Saavedra & EF529976 & EF529862 \\
\hline Sporophila simplex & LSUMZ B33437 & Peru: Cajamarca, Las Juntas, junction of Rios Tabacomasand Chinchipe & JN810149 & JN810542 \\
\hline Sporophila telasco & LSUMZ B32935 & Peru: Cajamarca, Las Juntas, junction of Rios Tabacomasand Chinchipe & JN810150 & JN810543 \\
\hline Sporophila torqueola & MBM 8476 & Honduras: Depto. Atlantida, La Ceiba, 15 km W & JN810151 & JN810544 \\
\hline Stephanophorus diadematus & AMNH DOT 9915 & Argentina: Buenos Aires, Partido Escobar & EU647992 & EU648053 \\
\hline Tachyphonus coronatus & AMNH DOT 2452 & $\begin{array}{l}\text { Argentina: Misiones Departamento San Ignacio, near border Parque Prov. Urugua-I, CA } 1 \text { KM W. Park Headquarters Ruta } \\
\text { Prov. } 19\end{array}$ & FJ799885 & JN810545 \\
\hline Tachyphonus cristatus & LSUMZ B9548 & Bolivia: Pando, Nicholas Suarez, $12 \mathrm{~km}$ by road S of Cojiba, $8 \mathrm{~km} \mathrm{~W}$ on road to Mucden & FJ799888 & JN810546 \\
\hline Tachyphonus delatrii & LSUMZ B11710 & Ecuador: Esmeraldas, El Placer & FJ799890 & JN810547 \\
\hline Tachyphonus luctuosus & LSUMZ B2279 & Panama: Darien, Cana on E slope Cerro Pirre & FJ799891 & JN810548 \\
\hline Tachyphonus phoenicius & AMNH DOT 4797 & Venezuela: Bolivar, Cerro Guanay & FJ799893 & JN810549 \\
\hline Tachyphonus rufiventer & LSUMZ B3629 & Peru: Loreto, S bank Rio Maranon, along Rio Samiria, Est. Biol. Pithecia, Base Tacsha Cocha & FJ799895 & JN810550 \\
\hline Tachyphonus rufus & LSUMZ B6668 & Bolivia: Santa Cruz, Rio Tucavaca & FJ799896 & JN810551 \\
\hline Tachyphonus surinamus & LSUMZ B4795 & Peru: Loreto, S Rio Amazonas, ca. 10 km SSW Rio Napo & EU647923 & EU647959 \\
\hline Tangara argyrofenges & ANSP 4482 & Ecuador: Zamora-Chinchipe, Panguri, ca. 12 km NE San Francisco del Vergel & AY383104 & JN810552 \\
\hline Tangara arthus & LSUMZ B22591 & Bolivia: La Paz, B. Saavedra, 83 km by road E Charazani, Cerro Asunta Pata & AY383106 & EU648055 \\
\hline Tangara callophrys & LSUMZ B34961 & Ecuador: Napo, ca. 20 km SSW Loreto & AY383107 & EU648056 \\
\hline Tangara cayana & LSUMZ B15414 & Bolivia: Santa Cruz, Serrania De Huanchaca, 45 km E Florida & AY383108 & JN810553 \\
\hline Tangara chilensis & MVZ 169699 & Peru: Dept. Cajamarca, 1 mi N San Jose de Lourdes, Cordillera del Condor & AY383110 & EU648058 \\
\hline
\end{tabular}


Locality

Tangara chrysotis

Tangara cucullata

Tangara cyanicollis

Tangara cyanocephala

Tangara cyanocephala 2

Tangara cyanoptera

Tangara cyanotis

Tangara cyanoventris

Tangara desmares

Tangara dowii

Tangara fastuosa

Tangara florida

Tangara fucosa

Tangara guttata

Tangara gyrola

Tangar heinei

Tangara icterocephal

Tangara inornata

Tangara johannae

Tangara labradorides

Tangara larvata

Tangara lavinia

Tangara mexicana

Tangara meyerdeschauenseei

Tangara nigrocincta

Tangara nigroviridis

Tangara palmeri

Tangara parzudaki

Tangara phillipsi

Tangara preciosa

Tangara punctata

Tangara punctata

Tangara ruficervix

Tangara rufigenis

Tangara rufigula

Tangara schrankii

Tangara seledon

Tangara varia

Tangara vassorii

Tangara velia

Tangara viridicollis

Tangara vitriolina

Tangara xanthocephala

Tangara xanthogastra

Tersina viridis

Tersina viridis 2

Thlypopsis fulvicep

Thlypopsis fulviceps

Thlypopsis ornata

Thlypopsis pectoralis

Thlypopsis ruficeps

Thlypopsis sordida

Thraupis abbas

\section{LSUMZ B34927}

STRI SV-TCU2

LSUMZ B15352

FMNH 427278

FMNH 427279

LSUMZ B7436

LSUMZ B22708

FMNH 311036

FMNH 395478

LSUMZ B16020

FMNH 427276

LSUMZ B34982

LSUMZ B1398

SUMZ B2190

SSUMZ B2190

LSUMZ B22850

SUMZ B16032

LSUMZ B16032

LSUMZ B28766

LSUMZ B29956

LSUMZ B32686

LSUMZ B34909

LSUMZ B34987

LSUMZ B18465

LSUMZ B43111

LSUMZ B9758

LSUMZ B1627

LSUMZ B11999

LSUMZ B30007

AMNH 821010

CUMV 50646

SUMZ 334931

LSUMZ B35552

LSUMZ B35552

LSUMZ B8190

UMMZ 134758

LSUMZ B11930

LSUMZ B34932

LSUMZ B16942

LSUMZ B28010

LSUMZ B1711

FMNH 390060

LSUMZ B8090

LSUMZ B34921

LSUMZ B34922

LSUMZ B34934

LSUMZ B9680

SUMZ B14819

MVZ 178272

IVZ 178272

LSUMZ B8075

LSUMZ B3608

FMNH 430081

MBM 7056
Ecuador: Napo, ca. $40 \mathrm{~km}$ NNE Ten

St. Vincent: Cumberland Valley

Bolivia: Santa Cruz, Serrania De Huanchaca, 45 km E Florida

Brazil: Pernambuco, Taquaritinga

Brazil: Pernambuco, Taquariting

Venezuela: Amazonas, Cerro de la Neblina Camp VII

Bolivia: La Paz, B. Saavedra, 83 km by road E Charazani, Cerro Asunta Pata

Brazil: Rio de Janeiro, Itatiaia, Fazenda de Serra

Brazil: Sao Paulo, Boracia

Costa Rica: Herredia, 4 km SE Virgen del Socorro

Brazil: Alagoas, Ibateourara Envenho Ceimba, Usina Serra Grande

Ecuador: Esmeraldas, $2 \mathrm{~km}$ W Alto Tambo

Panama: Darien, ca. 9 km NW Cana on slopes Cerro Pirre

Panama. Darien, Ca. 9 km NW Cana on slo

Bolivia: La Paz, B. Saavedra, $83 \mathrm{~km}$ by road E Charazani, Cerro Asunta Pata

Ecuador: Pichincha, $5 \mathrm{~km}$ S Nanegalito

Costa Rica: Herredia, 4 km SE Virgen del Socorro

Panama: Colon, Achiote Road, ca. 2 km bridge at Rio Providencia

Ecuador: Imbabura, ca. $20 \mathrm{~km}$ N Pedro Vicente Maldonado

Peru: Cajamarca, Quebrada Las Palmas, ca. 13 km WSW Chontall

Ecuador: Pichincha, $15 \mathrm{~km}$ N Pedro Vicente Maldonado

Ecuador: Esmeraldas, $30 \mathrm{~km}$ SE San Lorenzo

Bolivia: Santa Cruz, Velasco, Parque Nacional Noel Keonpff Mercado 86 km ESE of Florida Peru: Dept. Puno, $9.5 \mathrm{~km} \mathrm{~N}$ of Sándia

Bolivia: Pando, Nicholas Suarez, $12 \mathrm{~km}$ by road S of Cojiba, $8 \mathrm{~km} \mathrm{~W}$ on road to Mucden

Peru: Pasco, Santa Cruz, about 9 km SSE Oxapampa

Ecuador: Esmeraldas, El Placer

Ecuador: Esmeraldas, ca. $2.7 \mathrm{~km}$ E Alto Tambo

Peru: Dept. Huanuco, Cerros del Sira

Uruguay: Cerro Largo, Rio Yaguaron

Ecuador: Napo, ca. $40 \mathrm{Km}$ NNE Ten

Brazil: Fazenda Morelandia, 8 km N de Santa barbara, do Para

Peru: Pasco, Playa Pampa, ca. 8 km NW Cushi on trail to Chaglla

Venezuela: Aragua, Rancho Grande, Lat

Ecuador: Esmeraldas, El Placer

ESW Loreto

Brazil: Sao Paulo, Salesopolis, E.B. Boraceia

Peru: Loreto, ca. $77 \mathrm{~km}$ WNW Contaman

Peru: Pasco, Santa Cruz, about 9 km SSE Oxapampa

Brazil: Rondonia, Cachoeeira Nazare, W bank Rio jiparan

Peru: Pasco, Playa Pampa, ca. 8 km NW Cushi on trail to Chaglla

Ecuador: Pichincha, Tumbaco, Avenal, Buena Ezperanza

Ecuador: Pichincha, $5 \mathrm{~km}$ SE Baeza

Bolivia: Pando, Nicholas Suarez, $12 \mathrm{~km}$ by road S of Cojiba, $8 \mathrm{~km} \mathrm{~W}$ on road to Mucden

Bolivia

Venezuela: Zulia, Cerro Pejochaina, Perija

Peru: Depto. Cajamarca, 1 mi N San José de Lourdes, Cordillera del Condor

Peru: Pasco, Playa Pampa, $8 \mathrm{~km}$ NW Cushi on trail to Chaglla

Peru: Huanuco, Quebrada Shugush, $30 \mathrm{~km}$ on Huanuco-La Union Rd

Peru: Cuzco, Paucartambo: San Pedro, $2460 \mathrm{~m}$

Peru: Loreto, Amazonas I. Pasto 80 km NE Iquitos $80 \mathrm{~m}$

Honduras: Depto. Copan, Copan Ruinas, 10 km ENE

AY383111 EU648059

AY383113 EU648060

AY383115 EU64806

AY383117

AY383116 EU64806

AY383119 EU64806

JN810152 -

AY383120 EU648065

AY383121 EU648066

AY383126 EU648070

AY383131 EU648071

AY383132 EU648072

AY383132 EU648072

AY383133 EU648073

AU648075

AY383136 EU648076

AY383139 EU648078

AY383140 EU648079

EU648080

AY383143 EU648081

AY383144 EU648082

AY383146 EU648083

AY383147 EU64808

JN810153

EU647994 EU648085

AY383148

$-$

EU648087

AY383150 EU64808

JN810154

AY383152 EU648089

AY383153 EU648090

AY383154 EU64809

AY383155 EU648092

AY383156 EU648093

AY383158 EU648094

AY383159 EU648095

AY383160 EU648096

AY383161 EU648097

AY383162 EU648098

AF006255

AF447309

JN810155

JN810157 JN810555

JN810158 JN810556

JN810159 JN810557

JN810558

EU647996 EU64809

(continued on next page) 


\begin{tabular}{|c|c|c|c|c|}
\hline & Voucher/Sample Number $^{\mathrm{a}}$ & Locality & Cyt $b$ & ND2 \\
\hline Thraupis cyanocephala & FMNH 433897 & Peru: Cuzco, Paucartambo, La Esperanza, 39 km (road) NE Paucartambo, $2850 \mathrm{~m}$ & EU647998 & EU648101 \\
\hline Thraupis cyanoptera & FMNH 395473 & Brazil: Sao Paulo, Boracia & EU647999 & EU648102 \\
\hline Thraupis episcopus & FMNH 433904 & Peru: Madre de Dios, Moskitania, 13.4 km NNW Atalaya, I bank Alto Madre de Dios, 480 m & EU648000 & EU648103 \\
\hline Thraupis ornata & FMNH 395474 & Brazil: Sao Paulo, Boracia & EU648001 & EU648104 \\
\hline Thraupis palmarum & FMNH 427254 & Brazil: Alagoas, Ibateouara, Envenho Ceimba, Usina Serra Grande & EU648002 & EU648105 \\
\hline Thraupis sayaca & FMNH 334600 & Bolivia: El Beni, Trinidad, $10 \mathrm{~km} \mathrm{SW}$ & EU648003 & EU648106 \\
\hline Tiaris bicolor & MVZ 179402 & captive bird & AF489899 & - \\
\hline Tiaris bicolor 2 & BMNH JK95001 & Bahamas: Long Island & - & AF290115 \\
\hline Tiaris canorus & STRI BH-TCN1 & Bahamas: Gape's Backyard & HQ153058 & HQ153078 \\
\hline Tiaris fuliginosus & LSUMZ B12612 & Bolivia: Santa Cruz, Velasco, $50 \mathrm{~km}$ ESE of Florida, Arroyo del Encanto & AF489900 & EU648107 \\
\hline Tiaris obscurus & STRI BO-TOB22763 & Bolivia: Departmento La Paz; Prov. B. Saavedra; Cerro Asunta Pata & HQ153059 & HQ153081 \\
\hline Tiaris olivaceus & AMNH 25429 & Dominican Republic: Independencia, Parque Nacional Sierra de Baoruco, El Aceitillar, Alcoa Rd. & AF489901 & - \\
\hline Tiaris olivaceus 2 & UMMZ 233813 & Dominican Republic: Independencia, Parque Nacional Sierra de Baoruco, El Aceitillar, Alcoa Rd. & - & AF447310 \\
\hline Trichothraupis melanops & AMNH DOT 2464 & Argentina: Misiones Departamento San Ignacio about 20 km SE San Ignacio & FJ799899 & JN810559 \\
\hline Urothraupis stolzmanni & ZMUC 120310 & Ecuador: Tungurahua, C Llanganates & JN810160 & JN810560 \\
\hline Volatinia jacarina & FMNH 394403 & Bolivia & AF489903 & AF290113 \\
\hline $\begin{array}{c}\text { Wetmorethraupis } \\
\text { sterrhopteron }\end{array}$ & FMNH 275691 & Peru: Amazonas & EU648004 & - \\
\hline Xenodacnis parina & LSUMZ B7760 & Ecuador: Azuay, $1 \mathrm{~km}$ W CJS Nacional de Recreacion, near MGR & AF006257 & EU647960 \\
\hline Xenospingus concolor & LSUMZ B5263 & Peru: Dept. Ica; 0.5 km E km 235 Pan-American Hwy & JN810161 & JN810561 \\
\hline \multicolumn{5}{|l|}{ Outgroup species } \\
\hline Pheucticus tibialis & LSUMZ B16050 & Costa Rica: Heredia Province & JX569837 & JX569838 \\
\hline Piranga ludoviciana & BMNH jk94-105 & USA: Montana, Missoula Co. & EF529998 & AF290109 \\
\hline Cardinalis cardinalis & BMNH X7320 & USA: Minnesota & EF530007 & EF529902 \\
\hline Passerina ciris & LSUMZ B5694 & USA: Louisiana, Cameron Parish & AF301459 & EF529883 \\
\hline
\end{tabular}

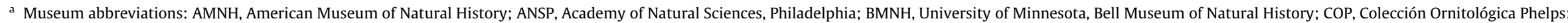

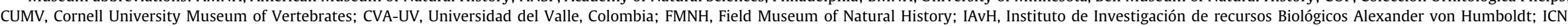

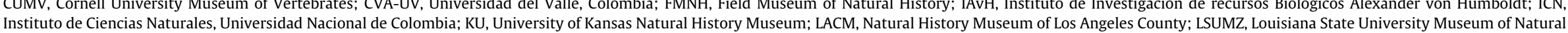

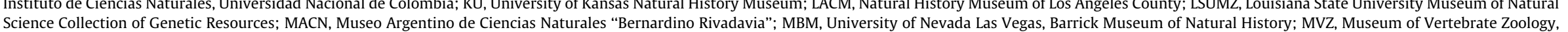

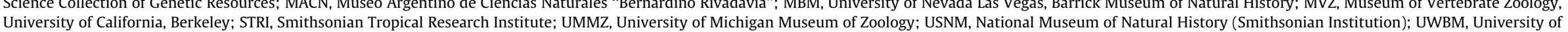
Washington, Burke Museum; ZMUC, Zoological Museum, University of Copenhagen. 
was computed simultaneously with 1000 bootstrap replicates, and run independently several times.

We conducted Bayesian analyses using BEAST v1.7.1 (Drummond et al., 2012) to produce an ultrametric tree. We analyzed the concatenated dataset containing all genes and taxa, partitioned as in the maximum likelihood analyses. We unlinked rate heterogeneity, base frequencies, and substitution rates across partitions. Models of evolution for each partition were inferred using jModelTest (Posada, 2008). Models were selected for the gene partitions using the AIC criterion (Akaike, 1973), and for each codon partition using the AICc, corrected for small sample size (Sugiura, 1978). In cases where the unimplemented TVM or TrN models were selected, we substituted the more general GTR model. All partitions used the GTR $+\mathrm{I}+\mathrm{G}$ model except for ND2 codon 3, RAG1 codon 3, ACO1-I9, FGB-I5, and MB-I2 partitions, which used the GTR + G model, and the RAG1 codon 2 partition, which used the HKY + I + G model. We used a Yule speciation model for tree shape, and an uncorrelated log-normal relaxed clock model with unlinked estimated rates across all partitions except for cyt $b$ (Drummond et al., 2006). We linked rates across the three cyt $b$ partitions, and used a substitution rate of 0.0105 mean substitutions per million years along each branch (Weir and Schluter, 2008). We used automatic optimization on MCMC operators, and ran analyses for a total of 200 million generations across four independent runs sampling every 5000 generations. We used Tracer v1.5 (Rambaut and Drummond, 2007) to assess convergence across independent runs and examine effective sample size values for all parameters. We also assessed convergence of the topology and clade stability using the online program Are We There Yet (Nylander et al., 2008). We discarded the first 20 million generations and 4000 trees for each run as a conservative burn-in, and combined both $\log$ and trees files using the program LogCombiner v1.7.1 (Drummond et al., 2012). We produced a maximum clade credibility tree using the BEAST program TreeAnnotator v1.7.1 (Drummond et al., 2012).

\subsection{Diversification analyses}

We compared the fit of five models of diversification for the entire clade (Thraupidae) and for each subfamily of tanagers with more than five species, as implemented in the $\mathrm{R}$ packages laser v2.3 (Rabosky, 2006) and TreePar v2.5 (Etienne et al., 2012). These models are: (1) a pure-birth model, which is a 1-parameter rate-constant model with no extinction (PB; Yule, 1924) (2) a birth-death model, which is a 2-parameter rate-constant model that infers speciation and extinction (BD; Kendall, 1948; Nee et al., 1994), (3) a 2-parameter rate-variable exponential-variant diversity-dependent model that assumes no extinction (DDX - E; Nee et al., 1992; Rabosky, 2006), (4) a 2-parameter rate-variable logistic-variant diversity-dependent model that assumes no extinction (DDL - E; Nee et al., 1992; Rabosky, 2006), and (5) a 3-parameter rate-variable diversity-dependent model which does estimate the rate of extinction (DDL + E; Etienne et al., 2012). For each clade, AIC $_{\mathrm{c}}$ scores were used to distinguish which model of diversification fit best. Models were considered to have a difference of fit if the difference in $\mathrm{AIC}_{\mathrm{c}}$ scores was two or greater (Burnham and Anderson, 2002). In addition, we created a lineage-through-time plot to get a more intuitive sense of tanager diversification. We also estimated the gamma statistic (Pybus and Harvey, 2000), which is a measure of rate constancy of lineage accumulation through time. The effect of incomplete taxon sampling on gamma statistic estimates was accounted for using the "mccr" test with 10,000 simulation replicates, and the test was run over 1000 trees randomly selected from the postburn-in posterior distribution of the BEAST analysis.
We also modeled diversification by treating the phylogeny as having been shaped by a mixture of macroevolutionary processes, using a novel Bayesian approach called BAMM (Bayesian Analysis of Macroevolutionary Mixtures, Rabosky et al., 2013; Rabosky and Matute, 2013; Rabosky, 2014). The model fits multiple diversification processes to various subtrees in the phylogeny, and uses reversible jump Markov Chain Monte Carlo to explore potential model space until the combination of processes with the highest likelihood is found. Shifts in diversification are detected automatically, with no a priori designations, and can occur at nodes or along branches, where the number of such shifts is assumed to follow a compound Poisson distribution. Importantly, rate constancy in diversification is not assumed, and therefore the phylogeny can be found to include any combination of constant-rate and rate-variable diversification models. We ran BAMM for fifty million generations, allowing for time-heterogeneous speciation rates, but holding extinction rates as time-constant. We accounted for incomplete taxon sampling analytically by supplying numbers of missing species per subfamily.

\section{Results}

\subsection{Sequence characteristics and gene properties}

The genes varied in their size, number of variable sites, and number of parsimony informative sites. For each marker, these data are as follows: cyt $b(1143,589,519)$, ND2 (1041, 677, 612), RAG1 (2891, 813, 385), MB-I2 (730, 357, 164), ACO1-I9 $(1104,705,383)$, and FGB-I5 $(586,338,168)$. Average uncorrected sequence divergences for all pairwise comparisons for each marker were as follows: cyt $b(10.5 \%)$, ND2 (15.8\%), RAG1 (1.30\%), MB-I2 (2.17\%), ACO1-I9 (3.48\%), and FGB-I5 (2.33\%). As expected, the different markers varied in their ability to resolve relationships among the species (Supplementary Figs. 1-6). Between the two mtDNA markers, ND2 recovered more nodes with $\geqslant 70 \%$ ML bootstrap support than cyt $b$ (57\% of nodes for ND2 vs. $46 \%$ for cyt $b$ ). Among nuclear markers, the protein-coding gene RAG1 and the sex-linked ACO1-I9 recovered more strongly supported nodes than the other markers. For both RAG1 and ACO1-I9, 46\% of all possible nodes were strongly supported, whereas the MB-12 gene tree recovered 24\% and the FGB-I5 gene tree recovered only $17 \%$.

\subsection{Phylogenetic analyses}

In general, the ML and Bayesian concatenated trees had similar topologies (Figs. 1-6). A few nodes were recovered with strong support in only one of the two analyses; however, none of these nodes strongly conflicted with those of the other analyses. Both analyses recovered a monophyletic Thraupidae with strong support (1.0 PP [posterior probability]; 100\% bootstrap). Thraupidae was also recovered in all of the individual gene trees except FBI5 (Supplementary Figs. 1-6). Within Thraupidae, 70\% of the nodes were strongly supported in the concatenated Bayesian analyses $(\mathrm{PP} \geqslant 0.95)$, and $66 \%$ of nodes were strongly supported in the concatenated ML analyses (bootstrap $\geqslant 70 \%$ ). Nodes with weaker support include some of the early nodes in the tree and nodes defining relationships among some recent species that are only weakly differentiated from each other (e.g., species within Geospiza, Camarhynchus, and Sporophila).

Our analyses revealed 13 strongly-supported nodes relatively early in the tree that define novel subgroups of tanagers (Fig. 1) that we designate as subfamilies. These clades are the deepest nodes in the tree that are supported by either PP $\geqslant 0.95$ or bootstrap $\geqslant 70 \%$. All are recovered in both Bayesian and ML topologies, 
Table 2

Species names, voucher numbers, localities, and GenBank accession numbers of nuclear sequences included in the study.

\begin{tabular}{|c|c|c|c|c|c|c|}
\hline Species & $\begin{array}{l}\text { Voucher/Sample } \\
\text { Number }^{1}\end{array}$ & Locality & FGB-I5 & MB-I2 & Rag 1 & ACO1-19 \\
\hline \multicolumn{7}{|l|}{ Ingroup species } \\
\hline Acanthidops bairdi & LSUMZ B16267 & $\begin{array}{l}\text { Costa Rica: San Jose, Cerro de la Muerte, Pan American Highway, } \\
\text { km } 113\end{array}$ & JN810162 & JN810297 & JN810562 & JN809918 \\
\hline Anisognathus somptuosus & LSUMZ B566 & Peru: Puno, Abra de Maruncunca, 10 km SW San Juan del Oro & JN810163 & JN810298 & JN810563 & JN809919 \\
\hline Bangsia arcaei & USNM B01412 & Panama & JN810164 & JN810299 & JN810564 & JN809920 \\
\hline Buthraupis wetmorei & LSUMZ B337 & Peru: Cajamarca, Cerro Chinguela, 5 km NE Sapalache & JN810166 & JN810301 & JN810566 & JN809922 \\
\hline Calochaetes coccineus & LSUMZ B6134 & Ecuador: Morona Santiago, W slope de Cutucci Yapitya & JN810167 & JN810302 & JN810567 & JN809923 \\
\hline Cnemathraupis eximia & LSUMZ B365 & Peru: Cajamarca, Cerro Chinguela, 5 km NE Sapalache & JN810165 & JN810300 & JN810565 & JN809921 \\
\hline Catamblyrhynchus diadema & FMNH 433908 & $\begin{array}{l}\text { Peru: Cuzco, Paucartambo, La Esperanza, } 39 \text { km (road) NE } \\
\text { Paucartambo, } 2850 \mathrm{~m}\end{array}$ & JN810169 & JN810304 & JN810569 & JN809925 \\
\hline Catamenia homochroa & LSUMZ B426 & Peru: Dept. Piura; Cruz Blanca; 33 rd km SW Huancabamba & JN810170 & JN810305 & JN810570 & JN809926 \\
\hline Charitospiza eucosma & LSUMZ B15356 & Bolivia: Santa Cruz, Serrania de Huanchaca, $45 \mathrm{~km}$ E Florida & JN810171 & JN810306 & JN810571 & JN809927 \\
\hline Chlorochrysa calliparaea & LSUMZ B8103 & Peru: Pasco, Playa Pampo, 8 k NW Cushi on trail to Chaglla & JN810172 & JN810307 & JN810572 & JN809928 \\
\hline Chlorochrysa phoenicotis & LSUMZ B34873 & Ecuador: Pichincha, $30 \mathrm{~km}$ Santo Domingo de los Colorados & JN810173 & JN810308 & JN810573 & JN809929 \\
\hline Chlorophanes spiza & LSUMZ B2838 & Peru: Loreto, $1 \mathrm{~km} \mathrm{~N}$ Rio Napo, $157 \mathrm{~km}$ by river NNE Iquitos & JN810174 & JN810309 & JN810574 & JN809930 \\
\hline Chlorornis riefferii & LSUMZ B1859 & Peru: Pasco, Chumbre de Ollon, about 12 km E Oxapampa & JN810175 & JN810310 & JN810575 & JN809931 \\
\hline Chrysothlypis chrysomelas & LSUMZ B2189 & Panama: Darien, about 6 km NW Cana & JN810176 & JN810311 & JN810576 & JN809932 \\
\hline Cissopis leverianus & LSUMZ B1143 & Bolivia: La Paz, Rio Beni, ca. 20 km by river N Puerto Linares & JN810177 & JN810312 & JN810577 & JN809933 \\
\hline Cnemoscopus rubrirostris & LSUMZ B5624 & Peru: Amazonas, $30 \mathrm{~km}$ by road E Florida on road to Rioja & JN810178 & JN810313 & JN810578 & JN809934 \\
\hline Coereba flaveola & FMNH 397158 & $\begin{array}{l}\text { West Indies: Bahamas, Great Abaco, Little Harbour Lighthouse, } \\
3.75 \text { km SSW, Cay Road }\end{array}$ & HQ153061 & HQ153068 & HQ153083 & HQ153049 \\
\hline Conirostrum ferrugineiventre & FMNH 391984 & Peru: Cuzco, Urubamba, Pumahuanca & JN810179 & JN810314 & JN810579 & JN809935 \\
\hline Conirostrum margaritae & LSUMZ B7293 & Peru: Loreto, Amazonas I. Pasto 80 km NE Iquitos 80 m & JN810180 & JN810315 & JN810580 & JN809936 \\
\hline Conothraupis speculigera & LSUMZ B5127 & $\begin{array}{l}\text { Peru: Lambayeque, Las Pampas, km } 885 \text { Pan-American Hwy, } 11 \\
\text { road km from Olmos }\end{array}$ & JN810181 & JN810316 & JN810581 & JN809937 \\
\hline Coryphaspiza melanotis & LSUMZ B6826 & Bolivia: Beni, 3 k SW San Borja & JN810182 & JN810317 & JN810582 & JN809938 \\
\hline Coryphospingus cucullatus & FMNH 334587 & $\begin{array}{l}\text { Bolivia: Santa Cruz, Chiquitos, Purubi, } 30 \text { km S San Jose de } \\
\text { Chiquitos }\end{array}$ & JN810183 & JN810318 & JN810583 & JN809939 \\
\hline Creurgops dentatus & LSUMZ B580 & Peru: Puno, Abra de Maruncunca, 10 km SW San Juan del Oro & JN810184 & JN810319 & JN810584 & JN809940 \\
\hline Creurgops verticalis & LSUMZ B7974 & Peru: Pasco, Playa Pampa, 8 km NW Cushi on trail to Chaglla & JN810185 & JN810320 & JN810585 & JN809941 \\
\hline Cyanerpes cyaneus & FMNH 427305 & Brazil: Alagoas & JN810186 & JN810321 & JN810586 & JN809942 \\
\hline Cyanicterus cyanicterus & USNM B10923 & Guyana & JN810187 & JN810322 & JN810587 & JN809943 \\
\hline Cypsnagra hirundinacea & LSUMZ B15290 & $\begin{array}{l}\text { Bolivia: Santa Cruz, Velasco, Pre Parque Nacional Noel Kempff } \\
\text { Mercado, } 30 \mathrm{~km} \text { E Aserradero Moira }\end{array}$ & JN810188 & JN810323 & JN810588 & JN809944 \\
\hline Dacnis cayana & LSUMZ B15077 & Bolivia: Santa Cruz, Velasco, 13 km SW Piso Firme & JN810189 & JN810324 & JN810589 & JN809945 \\
\hline Diglossa lafresnayii & LSUMZ B351 & Peru: Cajamarca, Cerro Chinguela, $5 \mathrm{~km}$ NE Sapalache & JN810191 & JN810326 & JN810591 & JN809947 \\
\hline Diglossa cyanea & FMNH 430124 & Peru: Cuzco, Paucartambo, Pillahuata & JN810192 & JN810327 & JN810592 & JN809948 \\
\hline Diuca diuca & AMNH DOT 9942 & Argentina, Rio Negro, Departamento Norquinco & None & JN810328 & JN810593 & JN809949 \\
\hline Diuca speculifera & LSUMZ B22574 & Bolivia: La Paz, Zongo Valley, $7 \mathrm{~km}$ by road $\mathrm{N}$ of summit & JN810193 & JN810329 & JN810594 & JN809950 \\
\hline Dolospingus fringilloides & USNM B11981 & Guyana & JN810194 & JN810330 & JN810595 & JN809951 \\
\hline Donacospiza albifrons & KU 3316 & Paraguay: Misiones, 5 km NW Yabebyry, Estancia Santa Ana & JN810195 & JN810331 & JN810596 & JN809952 \\
\hline Dubusia castaneoventris & LSUMZ B3607 & $\begin{array}{l}\text { Peru: Huanuco, Quebrada Shugush, } 30 \mathrm{~km} \text { on Huanuco-La Union } \\
\text { Rd }\end{array}$ & JN810190 & JN810325 & JN810590 & JN809946 \\
\hline Dubusia taeniata & LSUMZ B7710 & Peru: Huanuco, Unchog Pass NNW Acomayo 3450 m & JN810196 & JN810332 & JN810597 & JN809953 \\
\hline Emberizoides herbicola & FMNH 395725 & $\begin{array}{l}\text { Brazil: Roraima, Fazenda Santa Cecilia, E bank Rio Branco, opposite } \\
\text { Boa Vista }\end{array}$ & JN810197 & JN810333 & JN810598 & JN809954 \\
\hline Embernagra platensis & FMNH 396034 & Bolivia & JN810198 & JN810334 & JN810599 & JN809955 \\
\hline Eucometis penicillata & LSUMZ B6551 & Bolivia: Santa Cruz, Rio Quizer & JN810199 & JN810335 & JN810600 & JN809956 \\
\hline Euneornis campestris & FMNH 331119 & Jamaica: Portland, Hollywell Park & HQ153062 & HQ153069 & HQ153084 & HQ153050 \\
\hline Gubernatrix cristata & MACN 68379 & $\begin{array}{l}\text { Argentina: Prov. Buenos Aires; Salinera Universal, Salina de Piedra, } \\
\text { Cardenal Cagliero, Partido de Patagones }\end{array}$ & JN810200 & JN810336 & JN810601 & JN809957 \\
\hline Haplospiza rustica & FMNH 433797 & $\begin{array}{l}\text { Peru: Cuzco, Paucartambo, La Esperanza, } 39 \text { km (road) NE } \\
\text { Paucartambo }\end{array}$ & JN810201 & JN810337 & JN810602 & JN809958 \\
\hline Haplospiza unicolor & FMNH 395462 & Brazil: Sao Paulo, Boracia & JN810202 & None & None & None \\
\hline Hemispingus atropileus & LSUMZ B1889 & Peru: Pasco, Chumbre de Ollon, about 12 km E Oxapampa & JN810203 & JN810338 & JN810603 & JN809959 \\
\hline Hemispingus melanotis & FMNH 430079 & Peru: Cuzco, Paucartambo: San Pedro & JN810204 & JN810339 & JN810604 & JN809960 \\
\hline Hemispingus rufosuperciliaris & LSUMZ B3566 & Peru: Huanuco, base of bosque Zapatagocha above NE Acomayo & JN810205 & JN810340 & JN810605 & JN809961 \\
\hline Hemispingus superciliaris & FMNH 433858 & $\begin{array}{l}\text { Peru: Cuzco, Paucartambo, La Esperanza, } 39 \text { km (road) NE } \\
\text { Paucartambo }\end{array}$ & JN810206 & JN810341 & JN810606 & JN809962 \\
\hline Hemispingus verticalis & LSUMZ B320 & Peru: Cajamarca, Cerro Chingueal, 5 km NE Sapalache & JN810207 & JN810342 & JN810607 & JN809963 \\
\hline Hemithraupis flavicollis & LSUMZ B5102 & $\begin{array}{l}\text { Peru: Loreto, S Rio Amazonas, ca. } 10 \mathrm{~km} \mathrm{SSW} \text { mouth Rio Napo on E } \\
\text { bank Quebrada Vainilla }\end{array}$ & JN810208 & JN810343 & JN810608 & JN809964 \\
\hline Heterospingus xanthopygius & LSUMZ B2324 & Panama: Darien, Cana on E slope Cerro Pirre & JN810209 & JN810344 & JN810609 & JN809965 \\
\hline Idiopsar brachyurus & LSUMZ B22571 & Bolivia: La Paz, Zongo Valley, 7 km by road $\mathrm{N}$ of summit & JN810210 & JN810345 & JN810610 & JN809966 \\
\hline Incaspiza ortizi & LSUMZ B10382 & Peru: Cajamarca, above Limon Pampa between Calenda and Balsan & JN810211 & JN810346 & JN810611 & JN809967 \\
\hline Incaspiza pulchra & LSUMZ B10387 & Peru: Ancash, just out of Huaylash toward Sucre & JN810212 & JN810347 & JN810612 & JN809968 \\
\hline Iridophanes pulcherrimus & MVZ 169712 & $\begin{array}{l}\text { Peru: Dept. Cajamarca, } 1 \mathrm{mi} \text { N San Jose de Lourdes, Cordillera del } \\
\text { Condor }\end{array}$ & JN810213 & JN810348 & JN810613 & JN809969 \\
\hline Iridosornis analis & LSUMZ B1706 & Peru: Pasco, Santa Cruz, about 9 km SSE Oxapampa & JN810214 & JN810349 & JN810614 & JN809970 \\
\hline Iridosornis jelskii & FMNH 430099 & Peru: Cuzco, Paucartambo: Pillahuata, $2460 \mathrm{~m}$ & JN810215 & JN810350 & JN810615 & JN809971 \\
\hline Lanio fulvus & LSUMZ B2694 & Peru: Loreto, $1 \mathrm{~km} \mathrm{~N}$ Rio Napo, $157 \mathrm{~km}$ by river NNE Iquitos & JN810216 & JN810351 & JN810616 & JN809972 \\
\hline Lophospingus griseocristatus & FMNH 334558 & Bolivia: Cochabamba, Cochabamba-Oruro Rd, km 29 & JN810217 & JN810352 & JN810617 & JN809973 \\
\hline Loxigilla violacea & AMNH 25433 & Dominican Republic: Independencia & HQ153063 & HQ153070 & HQ153085 & HQ153051 \\
\hline Loxigilla portoricensis & PR-LPO26 & Puerto Rico & EF567717 & None & None & None \\
\hline
\end{tabular}


Table 2 (continued)

\begin{tabular}{|c|c|c|c|c|c|c|}
\hline Species & $\begin{array}{l}\text { Voucher/Sample } \\
\text { Number }^{1}\end{array}$ & Locality & FGB-I5 & MB-I2 & Rag 1 & ACO1-I9 \\
\hline Loxipasser anoxanthus & FMNH 33107 & Jamaica: Surrey, Portland, Hollywell Park & HQ153064 & HQ153071 & HQ153086 & HQ153052 \\
\hline Melanodera xanthogramma & AMNH DOT 12115 & Argentina: Departamento Bariloche, Rio Negro & JN810218 & JN810353 & JN810618 & JN809974 \\
\hline Melanospiza richardsoni & SL-MRI2 & St. Lucia & EF567721 & None & EF567526 & None \\
\hline Melopyrrha nigra & FMNH 342954 & captive bird & HQ153065 & HQ153072 & HQ153087 & HQ153053 \\
\hline Nemosia pileata & LSUMZ B7295 & Peru: Loreto, Amazonas I. Pasto $80 \mathrm{~km}$ NE Iquitos $80 \mathrm{~m}$ & JN810219 & JN810354 & JN810619 & JN809975 \\
\hline Neothraupis fasciata & LSUMZ B13914 & Bolivia: Santa Cruz, Serrania de Huanchaca, 45 km E Florida & JN810220 & JN810355 & JN810620 & JN809976 \\
\hline Nephelornis oneilli & LSUMZ B8402 & Peru: Pasco, Millpo, E Tambo de vacas on Pozuzo-Chaglla trail & JN810221 & JN810356 & JN810621 & JN809977 \\
\hline Nesospiza acunhae & ITU35 & Inaccessible Island, Tristan da Cunha & JN810222 & JN810357 & JN810622 & JN809978 \\
\hline Oreomanes fraseri & LSUMZ B2069 & Peru: Lima, ca. 13 road km W Milloc & JN810223 & JN810358 & JN810623 & JN809979 \\
\hline Oryzoborus angolensis & FMNH 433798 & $\begin{array}{l}\text { Peru: Madre de Dios, Moskitania, } 13.4 \text { km NNW Atalaya, I bank } \\
\text { Alto Madre de Dios }\end{array}$ & JN810224 & JN810359 & JN810624 & JN809980 \\
\hline Oryzoborus crassirostris & FMNH 339668 & Venezuela: Sucre, Guraunos, 14 km SSE & None & JN810360 & None & JN809981 \\
\hline Parkerthraustes humeralis & LSUMZ B9328 & Boliva: Pando & JN810168 & JN810303 & JN810568 & JN809924 \\
\hline Paroaria capitata & UWBM JAG-1837 & Argentina: Corrientes & JN810225 & JN810361 & JN810625 & JN809982 \\
\hline Paroaria coronata & FMNH 394390 & Bolivia & JN810226 & JN810362 & JN810626 & JN809983 \\
\hline Paroaria dominicana & FMNH 392736 & $\begin{array}{l}\text { Brazil: Sergripe, Caninde do Sao Francisco, Curituba, Fazenda Porto } \\
\text { Belo }\end{array}$ & JN810227 & JN810363 & JN810627 & JN809984 \\
\hline Paroaria gularis & FMNH 323625 & Peru: Madre de Dios, Hacienda Amazonia & JN810228 & JN810364 & JN810628 & JN809985 \\
\hline Phrygilus carbonarius & AMNH DOT 10373 & Argentina: Neuquen, Departamento Anelo, Sierra Auca Mahuida & JN810229 & JN810365 & JN810629 & JN809986 \\
\hline Phrygilus dorsalis & LSUMZ B17176 & Argentina & JN810230 & JN810366 & JN810630 & JN809987 \\
\hline Phrygilus erythronotus & LSUMZ B103892 & Peru: Tacna, Tacna-Llave Rd, ca. 57 km NE Tarata & JN810231 & JN810367 & JN810631 & JN809988 \\
\hline Phrygilus fruticeti & MBM 5412 & Argentina: Jujuy, Tilcara, 18 km S & JN810232 & JN810368 & JN810632 & JN809989 \\
\hline Phrygilus gayi & MBM 6475 & Argentina: Tucuman, Amaicha del Valle 12 km S, 12 km E & JN810233 & JN810369 & JN810633 & JN809990 \\
\hline Piezorina cinerea & LSUMZ B5169 & $\begin{array}{l}\text { Peru: Lambayeque, Las Pampas, km } 885 \text { Pan-American Hwy, } 11 \\
\text { road km from Olmos }\end{array}$ & JN810234 & JN810370 & JN810634 & JN809991 \\
\hline Pipraeidea melanonota & LSUMZ B12070 & Ecuador: Pichincha, Mindo & JN810235 & JN810371 & JN810635 & JN809992 \\
\hline Poospiza alticola & ZMUC 116453 & Peru: Ancash, C. Blanc, Andavite, Rurichinc. & JN810236 & JN810372 & JN810636 & JN809993 \\
\hline Poospiza hispaniolensis & LSUMZ 24977 & captive bird & None & JN810373 & JN810637 & JN809994 \\
\hline Poospiza hypochondria & MBM 6482 & Argentina. Tucuman & JN810238 & JN810374 & JN810638 & JN809995 \\
\hline Poospiza cabanisi & CUMV 50661 & Uruguay: Cerro Largo; Rio Yaguaron & JN810237 & JN810375 & JN810639 & JN809996 \\
\hline Poospiza melanoleuca & MBM 5316 & Argentina: Salta, J.V. Gonzalez, 14 km NE & JN810239 & JN810376 & JN810640 & JN809997 \\
\hline Poospiza ornata & AMNH DOT 9515 & locality unknown & JN810240 & JN810377 & JN810641 & JN809998 \\
\hline Poospiza torquata & KU 2838 & Paraguay: Presidente Hayes, Campo Largo, $5 \mathrm{~km} \mathrm{~S}$ & JN810241 & JN810378 & JN810642 & JN809999 \\
\hline Poospiza whitii & LSUMZ B6573 & Bolivia: Santa Cruz, $2.5 \mathrm{~km} \mathrm{~N}$ Tambo & JN810242 & JN810379 & JN810643 & JN810000 \\
\hline Porphyrospiza caerulescens & LSUMZ B13862 & Bolivia: Santa Cruz; Serrania de Huanchaca, $45 \mathrm{~km}$ E Florida & JN810243 & JN810380 & JN810644 & JN810001 \\
\hline Pyrrhocoma ruficeps & MVZ 165617 & Paraguay: Dept. Itapu, El Tirol, $19.5 \mathrm{~km}$ by road NNE Encarnacion & JN810244 & JN810381 & JN810645 & JN810002 \\
\hline Ramphocelus carbo & FMNH 430084 & Peru: Cuzco, Paucartambo: San Pedro & JN810245 & JN810382 & JN810646 & JN810003 \\
\hline Ramphocelus passerinii & MBM 4358 & Nicaragua, La Luz near Wani on Rio Uli & JN810246 & None & None & None \\
\hline Ramphocelus sanguinolentus & FMNH 343376 & $\begin{array}{l}\text { Mexico: Veracruz, El Bastonal, } 3 \text { km S, } 3 \text { km E, Sierra de Santa } \\
\text { Martha }\end{array}$ & JN810247 & JN810383 & JN810647 & JN810004 \\
\hline Rhodospingus cruentus & LSUMZ B5184 & $\begin{array}{l}\text { Peru: Lambayeque, Las Pampas, km } 885 \text { Pan-American Hwy, } 11 \\
\text { road km from Olmos }\end{array}$ & JN810248 & JN810384 & JN810648 & JN810005 \\
\hline Rowettia goughensis & GB18 & Gough Island & JN810249 & JN810385 & JN810649 & JN810006 \\
\hline Saltator atriceps & FMNH 343357 & Mexico: Veracruz & JN810250 & JN810386 & JN810650 & JN810007 \\
\hline Saltator atricollis & LSUMZ B15381 & Bolivia: Santa Cruz & JN810251 & JN810387 & JN810651 & JN810008 \\
\hline Saltator atripennis & ANSP 3491 & Ecuador: Azuay & JN810252 & JN810388 & JN810652 & JN810009 \\
\hline Saltator aurantiirostris & UWBM 54506 & Argentina: Tucuman & JN810253 & JN810389 & JN810653 & JN810010 \\
\hline Saltator cinctus & LSUMZ B6233 & Ecuador: Prov. Morona-Santiago & JN810254 & JN810390 & JN810654 & JN810011 \\
\hline Saltator coerulescens & UWBM GAV817 & Argentina: Corrientes & JN810255 & JN810391 & JN810655 & JN810012 \\
\hline Saltator grossus & LSUMZ B16063 & Costa Rica: Herredia & JN810256 & JN810392 & JN810656 & JN810013 \\
\hline Saltator maximus & FMNH 433810 & Peru: Cuzco, Paucartambo, Consuelo, 15.9 km SW Pilcopata & JN810257 & JN810393 & JN810657 & JN810014 \\
\hline Saltator nigriceps & LSUMZ B183 & Peru: Piura & JN810258 & JN810394 & JN810658 & JN810015 \\
\hline Saltator rufiventris & LSUMZ B106750 & Bolivia: Cochabamba & JN810259 & JN810395 & JN810659 & JN810016 \\
\hline Saltator similis & UWBM 70491 & Argentina: Provincia de Corrientes, Corrientes, Manuel Derqui & JN810260 & JN810396 & JN810660 & JN810017 \\
\hline Saltator striatipectus & LSUMZ B449 & Peru: Lambayeque & JN810261 & JN810397 & JN810661 & JN810018 \\
\hline Saltatricula multicolor & MVZ 179401 & Luis F. Baptista aviary & JN810262 & JN810398 & JN810662 & JN810019 \\
\hline Schistochlamys melanopis & LSUMZ B9669 & $\begin{array}{l}\text { Bolivia: Pando, Nicholas Suarez, } 12 \mathrm{~km} \text { by road S of Cojiba, } 8 \mathrm{~km} \mathrm{~W} \\
\text { on road to Mucden }\end{array}$ & JN810263 & JN810399 & JN810663 & JN810020 \\
\hline Sericossypha albocristata & LSUMZ B5630 & Peru: Amazonas, $30 \mathrm{~km}$ by road E Florida on road to Rioja & JN810264 & JN810400 & JN810664 & JN810021 \\
\hline Sicalis citrina & LSUMZ B15400 & Bolivia: Santa Cruz, Serrania De Huanchaca, $45 \mathrm{~km}$ E Florida & JN810265 & JN810401 & JN810665 & None \\
\hline Sicalis luteola & FMNH 389274 & $\begin{array}{l}\text { Brazil: Roraima, Fazenda Santa Cecilia, E Bank Rio Branco, across } \\
\text { from boa Vista }\end{array}$ & JN810266 & JN810402 & JN810666 & JN810022 \\
\hline Sporophila albogularis & FMNH 392743 & Brazil: Alagoas, Piranhas, Fazenda Bela Vista & JN810267 & JN810403 & JN810667 & JN810023 \\
\hline Sporophila lineola & FMNH 390057 & Brazil: Rondonia, Cachoeeira Nazare, W bank Rio jiparana & JN810268 & JN810404 & JN810668 & JN810024 \\
\hline Stephanophorus diadematus & AMNH 9915 & Argentina: Buenos Aires, Partido Escobar & JN810269 & JN810405 & JN810669 & JN810025 \\
\hline Tachyphonus coronatus & AMNH DOT2452 & $\begin{array}{l}\text { Argentina: Misiones Departamento San Ignacio, near border } \\
\text { Parque Prov. Urugua-I, CA } 1 \text { KM W. Park Headquarters Ruta Prov. } \\
\text { 19, Latitude } 2551 \text { S Longitude } 05410 \mathrm{~W}\end{array}$ & JN810270 & JN810406 & JN810670 & JN810026 \\
\hline Tachyphonus cristatus & LSUMZ B9548 & $\begin{array}{l}\text { Bolivia: Pando, Nicholas Suarez, } 12 \mathrm{~km} \text { by road S of Cojiba, } 8 \mathrm{~km} \mathrm{~W} \\
\text { on road to Mucden }\end{array}$ & JN810271 & JN810407 & JN810671 & JN810027 \\
\hline Tachyphonus delatrii & LSUMZ B11710 & Ecuador: Esmeraldas, El Placer & JN810272 & JN810408 & JN810672 & JN810028 \\
\hline Tachyphonus luctuosus & LSUMZ B2279 & Panama: Darien, Cana on E slope Cerro Pirre & JN810273 & None & None & None \\
\hline Tachyphonus rufiventer & LSUMZ B3629 & $\begin{array}{l}\text { Peru: Loreto, S bank Rio Maranon, along Rio Samiria, Est. Biol. } \\
\text { Pithecia, Base Tacsha Cocha }\end{array}$ & JN810274 & JN810409 & JN810673 & JN810029 \\
\hline
\end{tabular}


Table 2 (continued)

\begin{tabular}{|c|c|c|c|c|c|c|}
\hline Species & $\begin{array}{l}\text { Voucher/Sample } \\
\text { Number }^{1}\end{array}$ & Locality & FGB-I5 & MB-I2 & Rag 1 & ACO1-I9 \\
\hline Tachyphonus surinamus & LSUMZ B4795 & Peru: Loreto, S Rio Amazonas, ca. 10 km SSW Rio Napo & JN810275 & JN810410 & JN810674 & JN810030 \\
\hline Tangara cayana & LSUMZ B15414 & Bolivia: Santa Cruz, Serrania De Huanchaca, 45 km E Florida & JN810276 & JN810411 & JN810675 & JN810031 \\
\hline Tangara cyanocephala & FMNH 427278 & Brazil: Pernambuco, Taquaritinga & JN810277 & JN810412 & JN810676 & JN810032 \\
\hline Tangara gyrola & LSUMZ B22850 & $\begin{array}{l}\text { Bolivia: La Paz, B. Saavedra, } 83 \text { km by road E Charazani, Cerro } \\
\text { Asunta Pata }\end{array}$ & JN810278 & None & None & None \\
\hline Tangara preciosa & CUMV 50646 & Uruguay: Cerro Largo, Rio Yaguaron & JN810279 & JN810413 & JN810677 & JN810033 \\
\hline Tangara punctata & LSUMZ B34931 & Ecuador: Napo, ca. $40 \mathrm{Km}$ NNE Tena & JN810280 & JN810414 & JN810678 & JN810034 \\
\hline Tangara vassorii & LSUMZ B1711 & Peru: Pasco, Santa Cruz, about 9 km SSE Oxapampa & None & JN810415 & JN810679 & JN810035 \\
\hline Tangara rufigula & LSUMZ B11930 & Ecuador: Esmeraldas, El Placer & JN810281 & None & None & None \\
\hline Tersina viridis & LSUMZ B9680 & $\begin{array}{l}\text { Bolivia: Pando, Nicholas Suarez, } 12 \mathrm{~km} \text { by road S of Cojiba, } 8 \mathrm{~km} \mathrm{~W} \\
\text { on road to Mucden }\end{array}$ & JN810282 & JN810416 & JN810680 & JN810036 \\
\hline Thlypopsis ornata & LSUMZ B8075 & Peru: Pasco, Playa Pampa, 8 km NW Cushi on trail to Chaglla & JN810283 & JN810417 & JN810681 & JN810037 \\
\hline Thlypopsis sordida & LSUMZ B7260 & Peru: Loreto, Amazonas I. Pasto 80 km NE Iquitos 80 m & JN810284 & JN810418 & JN810682 & JN810038 \\
\hline Thraupis bonariensis & FMNH 433891 & $\begin{array}{l}\text { Peru: Cuzco, Paucartambo, La Esperanza, } 39 \text { km (road) NE } \\
\text { Paucartambo }\end{array}$ & JN810285 & JN810419 & JN810683 & JN810039 \\
\hline Thraupis cyanocephala & FMNH 433897 & $\begin{array}{l}\text { Peru: Cuzco, Paucartambo, La Esperanza, } 39 \text { km (road) NE } \\
\text { Paucartambo, } 2850 \mathrm{~m}\end{array}$ & JN810286 & JN810420 & JN810684 & JN810040 \\
\hline Thraupis palmarum & FMNH 427254 & Brazil: Alagoas, Ibateouara, Envenho Ceimba, Usina Serra Grande & JN810287 & JN810421 & JN810685 & JN810041 \\
\hline Tiaris bicolor & MVZ 179402 & captive bird & HQ153066 & HQ153073 & HQ153088 & HQ153054 \\
\hline Tiaris olivaceus & AMNH 25429 & $\begin{array}{l}\text { Dominican Republic: Independencia, Parque Nacional Sierra de } \\
\text { Baoruco, El Aceitillar, Alcoa Rd. }\end{array}$ & HQ153067 & HQ153074 & HQ153089 & HQ153055 \\
\hline Trichothraupis melanops & UWBM 70274 & Argentina: Prov. Misiones; Posadas, 45 km N, 80 km E & JN810288 & JN810422 & JN810686 & JN810042 \\
\hline Urothraupis stolzmanni & ZMUC 120310 & Ecuador: Tungurahua, C Llanganates & JN810289 & JN810423 & None & JN810043 \\
\hline Volatinia jacarina & FMNH 392749 & Brazil, Alagoas, Piranhas, Fazenda Mecejana & JN810290 & JN810424 & JN810687 & JN810044 \\
\hline Xenodacnis parina & LSUMZ B7760 & Ecuador: Azuay, 1 km W CJS Nacional de Recreacion, near MGR & JN810291 & JN810425 & JN810688 & JN810045 \\
\hline Xenospingus concolor & LSUMZ B5263 & Peru: Dept. Ica; 0.5 km E km 235 Pan-American Hwy & JN810292 & JN810426 & JN810689 & JN810046 \\
\hline \multicolumn{7}{|l|}{ Outgroup species } \\
\hline Pheucticus tibialis & LSUMZ B16050 & Costa Rica: Heredia Province & JN810293 & JN810427 & JN810690 & JN810047 \\
\hline Passerina ciris & LSUMZ B5694 & USA: Louisiana, Cameron Parish & JN810294 & JN810428 & JN810691 & JN810048 \\
\hline Piranga ludoviciana & SDSU 2383 & USA: California, San Diego Co., Laguna Mts. & JN810295 & JN810429 & JN810692 & JN810049 \\
\hline Cardinalis cardinalis & BMNH X7320 & USA: Minnesota & JN810296 & JN810430 & JN810693 & JN810050 \\
\hline
\end{tabular}

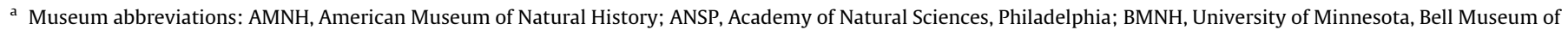

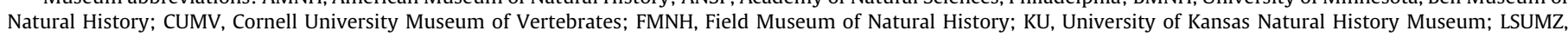

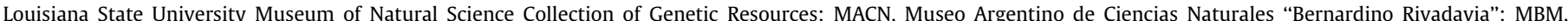

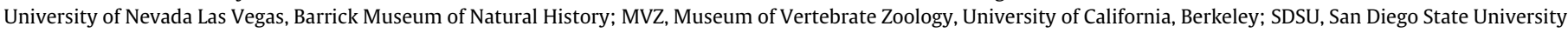

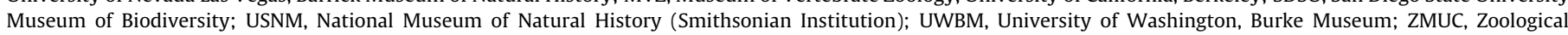
Museum, University of Copenhagen.

and most are also strongly supported by both. In addition, all but one of these subfamilies have significant support $(\geqslant 0.95 \mathrm{PP})$ in the species-tree analyses of Barker et al. (2013). Only two species (Catamblyrhynchus diadema and Charitospiza eucosma) did not cluster into one of these clades. Because of the distinctiveness of these two lineages, we place each into subfamilies as well. Although we identified these 15 major groups, we did not find strong support for relationships among them (Figs. 1-6).

Thraupidae now represents species that traditionally have belonged to four different avian families or subfamilies outside of Thraupidae (Paynter and Storer, 1970; Figs. 2-6): Catamblyrhynchinae (the Plushcap), Emberizinae (New World sparrows), Parulidae (New World warblers), and Cardinalinae (cardinal-grosbeaks). In most cases, these representatives of other groups do not cluster in a single place on our phylogenies. For example, the Emberizidae species that Sibley and Monroe (1990) transferred to the tanagers can be found in 10 of our 15 subfamilies (Figs. 2-6). Within Thraupidae, many traditional genera are not monophyletic in our phylogenies. Clements et al. (2013) currently recognizes 92 genera of tanagers. Of these, 43 are monotypic, indicating the historical difficulty in classifying tanagers. Of the remaining 49 genera, 17 are not monophyletic in our phylogenies (Figs. 2-6). These include well-known genera such as Tangara, Sporophila, and Thraupis. In fact, only three traditional genera that include more than six species are monophyletic in our phylogenies. In some cases (e.g., Saltator, Diuca, Phrygilus), species in a genus are found in multiple subfamilies of tanagers indicating that major revision at the genus level is warranted for the group.
Our genetic data revealed seven groups of species that are only weakly differentiated from their close relatives compared to other species of tanagers. These include (1) Geospiza, (2) Camarhynchus, (3) some species of Sporophila, (4) members of the Diglossa carbonaria superspecies complex (D. carbonaria, D. brunneiventris, $D$. humeralis, and D. gloriosa), (5) Nesospiza, (6) Idiopsar brachyurus/Diuca speculifera, and (7) Tangara phillipsi/T. argyrofenges/T. heinei. Some of these have been previously identified (e.g., Campagna et al., 2012; Mauck and Burns, 2009; Ryan et al., 2007; Sato et al., 1999), but others are shown for the first time as having little genetic divergence. All represent cases of plumage or morphological divergence accompanied by little genetic change, and some are identified as having an exceptional rate of speciation in our diversification analyses.

\subsection{Diversification analyses}

For the entire clade (Thraupidae), we found that the DDX model had the best fit (Table 3 ). We were not able to fit the $\mathrm{DDL}+\mathrm{E}$ due to computational limitations given the large number of species. The lineage-through-time plot showed a clear departure from the straight line expected under a constant-rate diversification scenario (Fig. 1). The gamma statistic and associated p-value (Table 3 ) indicate a significant slowdown in species accumulation through time. For seven of the ten tanager subfamilies tested, we found the best fit model to be a diversitydependent model, although the specific model varied (Table 3 ). For the remaining three subfamilies, one (Hemithraupinae) had 


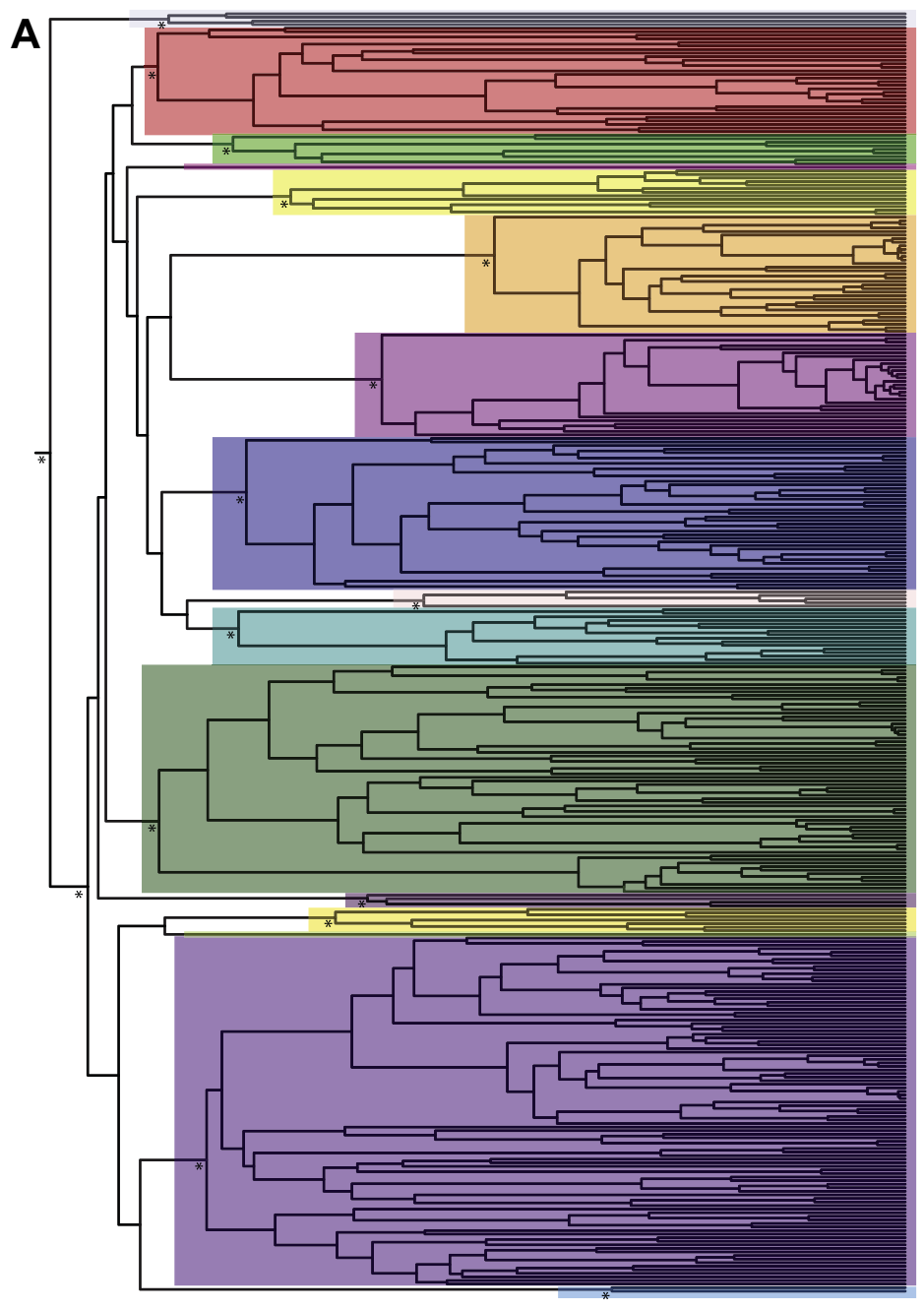

Cardinalidae Outgroups

Tachyphoninae

Hemithraupinae

Charitospizinae

Dacninae

Sporophilinae

Coerebinae

Poospizinae

Emberizoidinae

Saltatorinae

Diglossinae

Nemosiinae

Porphyrospizinae

Catamblyrhynchinae

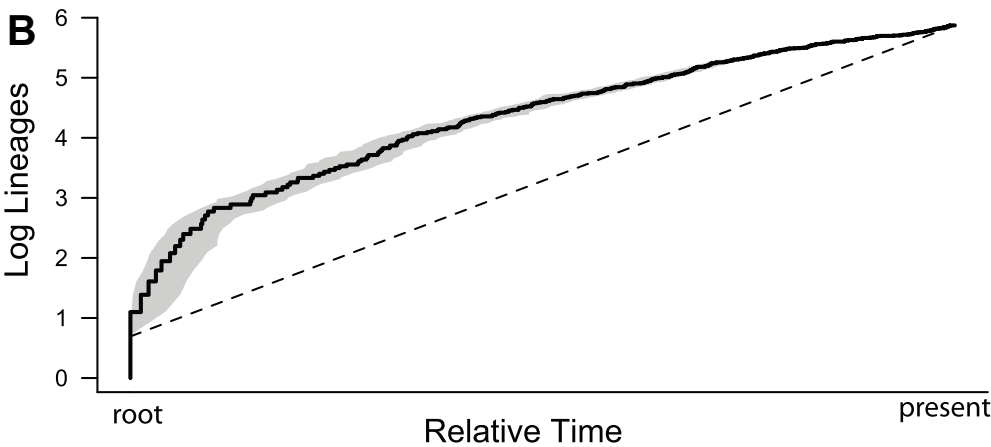

Orchesticinae

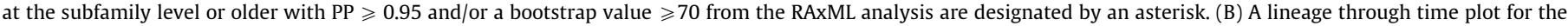

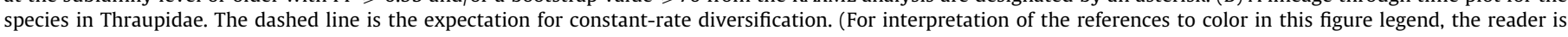
referred to the web version of this article.)

no difference in fit between the PB, DDL-E and DDX models (Table 3). Sporophilinae and Coerebinae best fit constant-rate models (Table 3). Gamma statistic results largely corroborated the model-fitting results; diversity-dependent model subfamilies have a significantly negative gamma statistic (Table 3), and constant-rate model subfamilies have non-significant, positive gamma statistics (Table 3). The exceptions are Hemithraupinae and Porphyrospizinae, with non-significant, negative gamma statistics, likely due to the small numbers of species in each clade and low statistical power.
We found strong support for multiple diversification rate shifts across the tanager phylogeny (Fig. 7). Although the diversification shift location was not always found on the same branch, we detected a rate shift along one of the branches leading up to the Darwin's Finch radiation in $92 \%$ of the post-burn-in posterior distribution of the BAMM results. In the entire post-burn-in posterior distribution, another shift occurred within Sporophilinae. Overall, three processes were found to most likely govern diversification in the tanagers: a background model of exponentially declining speciation rates for the entire tanager phylogeny, with 
A. Charitospizinae

Charitospiza eucosma $\psi$

B. Catamblyrhynchinae

Catamblyrhynchus diadema $\Phi$

C. Orchesticinae

$\frac{1}{100}$ C Parkerthraustes humeralis $\xi$

\section{Nemosiinae}

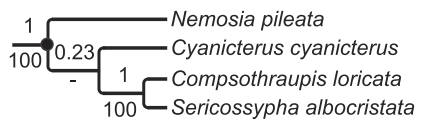

H. Dacninae

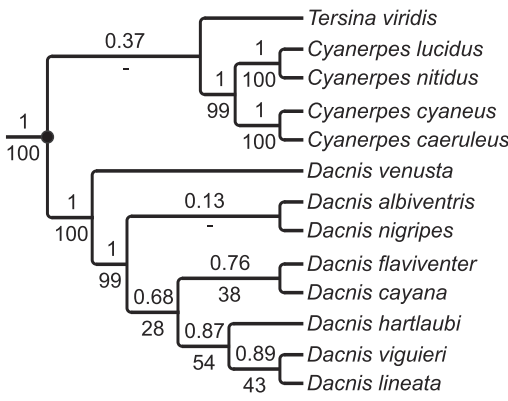

I. Saltatorinae

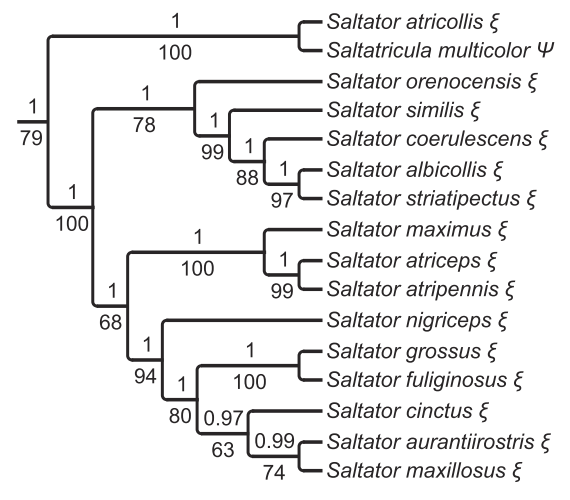

E. Emberizoidinae

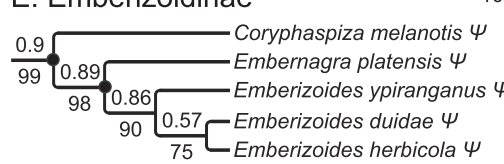

J. Coerebinae

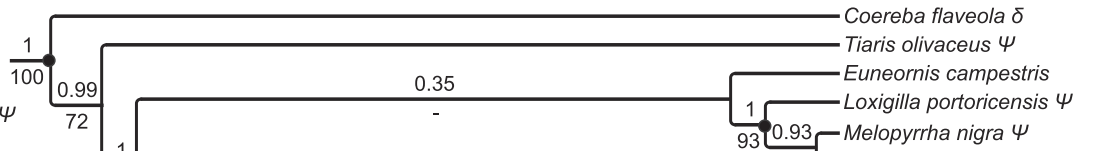

${ }_{83}$ Loxigilla violacea $\psi$

- Loxipasser anoxanthus $\psi$

97

Tiaris canorus $\psi$

Loxigilla noctis $\psi$

Tiaris bicolor $\psi$

F. Porphyrospizinae

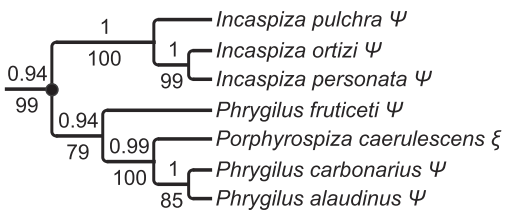

G. Hemithraupinae

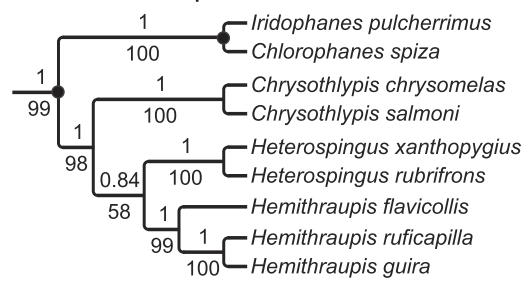

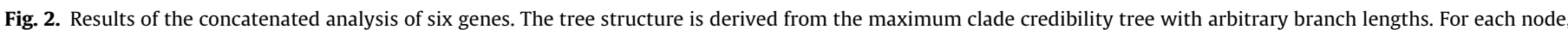

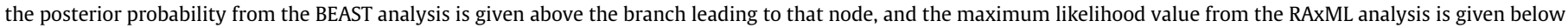

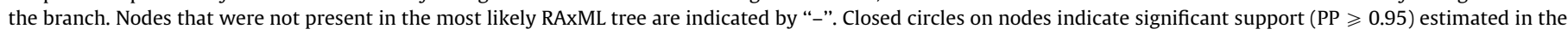

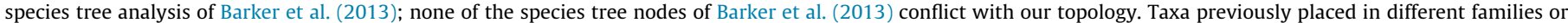

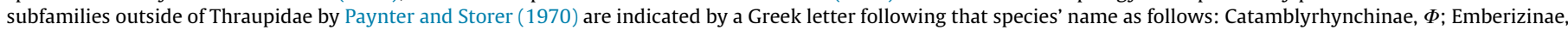

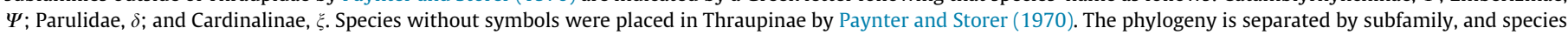

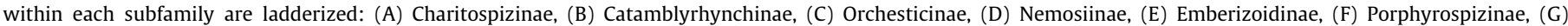
Hemithraupinae, (H) Dacninae, (I) Saltatorinae, (J) Coerebinae.

shifts to a new model of declining speciation rates within Sporophilinae and a model of near constant rate for Darwin's Finches. The initial rate of speciation found for both radiations is over three times the initial speciation rate of the background tanager process of exponential decline in speciation rates.

\section{Discussion}

\subsection{Tanagers as a model for studying character evolution}

The lack of a monophyletic Thraupidae has hindered the study of character evolution and diversification in the group. Thus, despite the fact that tanagers encompass a major portion of overall songbird diversity, tanagers are less well known than other better characterized, but smaller, groups of birds such as hummingbirds
(335 species) and parrots (345 species). Here, we provide a robust, species-level of phylogeny of tanagers that allows for the study of character evolution in the group. Previous attempts at tanager phylogenies (e.g., Bleiweiss, 2008; Burns, 1997; Fjeldså and Rahbek, 2006; Jetz et al., 2012; Sibley and Ahlquist, 1990; Weir et al., 2009) were missing key taxa, relied on sparse taxon sampling, or assumed genus-level monophyly. Our trees have numerous topological differences when compared to these earlier phylogenies. Therefore, other studies that relied on these earlier phylogenies to make interpretations about biogeography, evolution, behavior, or ecology should be reassessed in light of the new topologies presented here. Now that a monophyletic Thraupidae has been defined and a robust phylogeny provided, analyses of character evolution and biogeography can be pursued. In particular, tanagers appear to be a model system to study convergence. Different bill 


\section{A. Tachyphoninae}

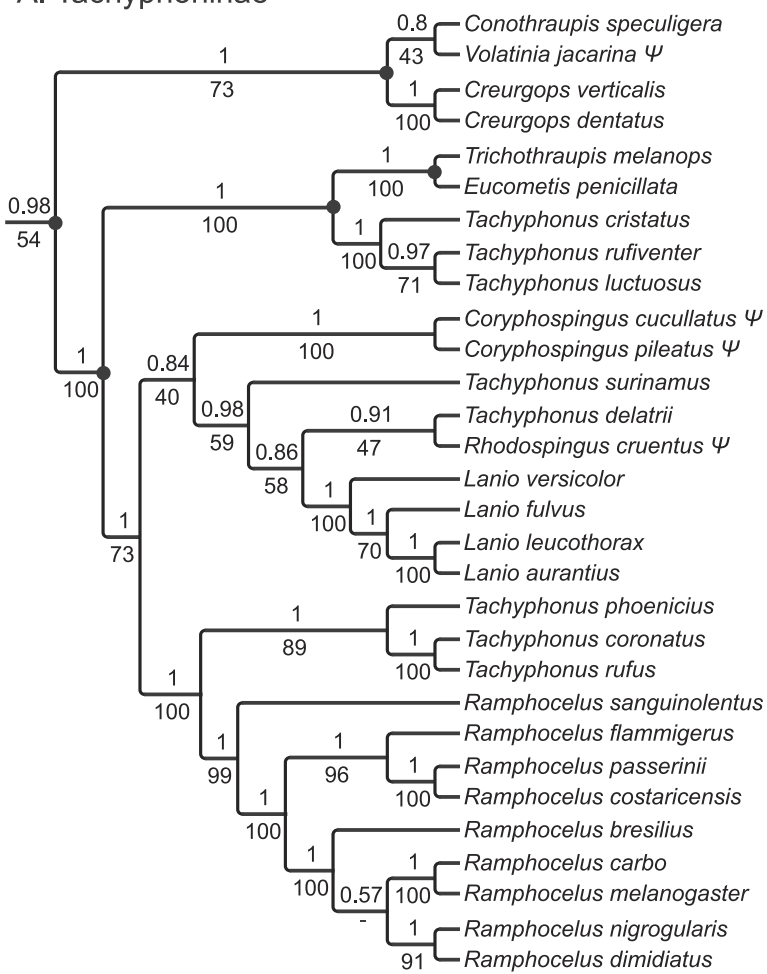

B. Sporophilinae

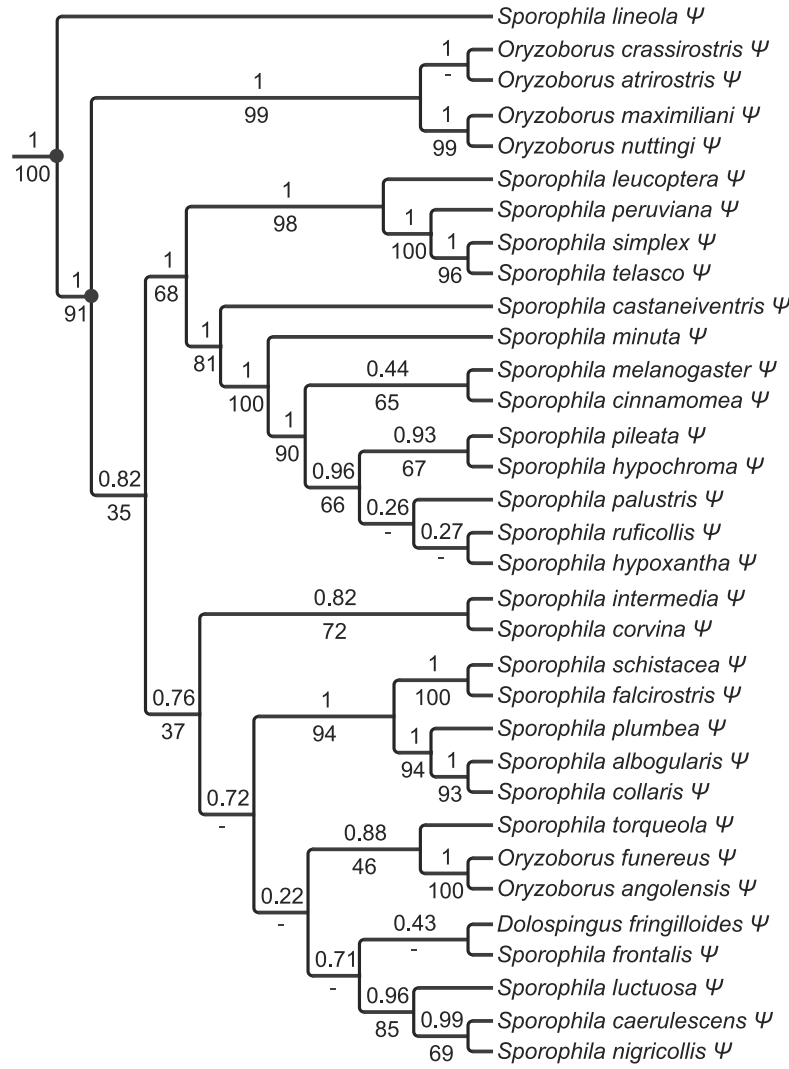

Fig. 3. Results of the concatenated analysis of the six genes for the subfamilies (A) Tachyphoninae and (B) Sporophilinae. Labeling and format as in Fig. 2.

types, foraging strategies, and ecomorphs have evolved repeatedly across the phylogeny. For example, the seed-eating finch-billed species that were previously classified in Emberizidae are spread across our phylogeny, indicating convergence of bill types across the group. In addition, nectar-feeding tanagers (Burns et al., 2003) occur in multiple places across the phylogeny. These and other specific examples are presented below in our discussion of tanager subfamilies (Section 4.3), and more rigorous comparative analyses are ongoing (e.g., Shultz and Burns, 2013).

\subsection{Diversification of tanagers}

The different approaches that we employed all generally led to the same finding that tanagers underwent an initial rapid burst in diversification, followed by a subsequent slowdown. This corroborates the results of Barker et al. (2013), who showed that Thraupidae exhibited a rate of diversification that was almost $40 \%$ higher than the average rate for the 9-primaried oscine clade, to which tanagers belong. Though Phillimore and Price (2008) showed that a negative gamma statistic could result from large clades operating under constant-rate diversification, due to stochasticity in the timing of speciation events, the gamma statistic for tanagers is more negative than any gamma generated in their simulations, lending confidence to a truly diversity-dependent pattern of diversification in tanagers. This pattern is largely paralleled across subfamilies within tanagers, as shown by the best-fitting models and significantly negative gamma statistics (Table 3 ).

Two subfamilies, however, show increased diversification rates over the background rate. Coerebinae, which includes the Darwin's finches, exhibits an increased rate of diversification with an overall signature of constant-rate diversification. This clade may exhibit a different pattern of diversification from the others due to both extrinsic and intrinsic factors (Rabosky, 2010). One the one hand, increased speciation rates may have been promoted by geographic isolation and ecological release across the Galapagos Islands. On the other, this lineage may exhibit intrinsic evolvability (Burns et al., 2002; Mallarino et al., 2012). Sporophilinae also exhibits a different signature of diversification, with a best-fit constant-rate model when comparing discrete models, and an increase in diversification rate compared to the tanager background rate in the BAMM analysis. Overall, the speciation rate appears to be declining in Sporophilinae. This could be because Sporophilinae is older than the Darwin's finch radiation, and thus may have reached a stage of ecological limits to diversification, whereas the Darwin's finches have not.

Darwin's finches are a classic example of adaptive radiation, and evolution within species in the group is well characterized (Grant, 1999; Grant and Grant, 2008). However, few studies have provided the comparative perspective needed to interpret the phylogenetic context of this adaptive radiation (Burns et al., 2002; Mallarino et al., 2012). In this study, we have quantified the diversification rate of Darwin's Finches in the context of diversification of their closest relatives for the first time. We find that their rate of diversification is exceptional, even when compared to the overall rapid rate of diversification found within tanagers. In addition, we find that the rate of diversification of some seedeaters in Sporophila is comparable to that of Darwin's finches. Both lineages are composed of primarily finch-billed forms. Price (2011) suggested that finch-billed lineages might be more prone to higher rates of diversification than other avian ecotypes. Although many other clades of tanagers contain finch-billed species, our finding that the two clades with the highest rates of diversification contain finch-billed forms is consistent with Price's (2011) hypothesis.

With the inclusion of diversification mixture models, we now have much greater ability to investigate the diversification history of large clades. The BAMM approach brings two improvements to existing methods: freedom from designating groups a priori, and the ability to treat a large phylogeny as a multi-process phenomenon (Rabosky et al., 2013; Rabosky and Matute, 2013; Rabosky, 


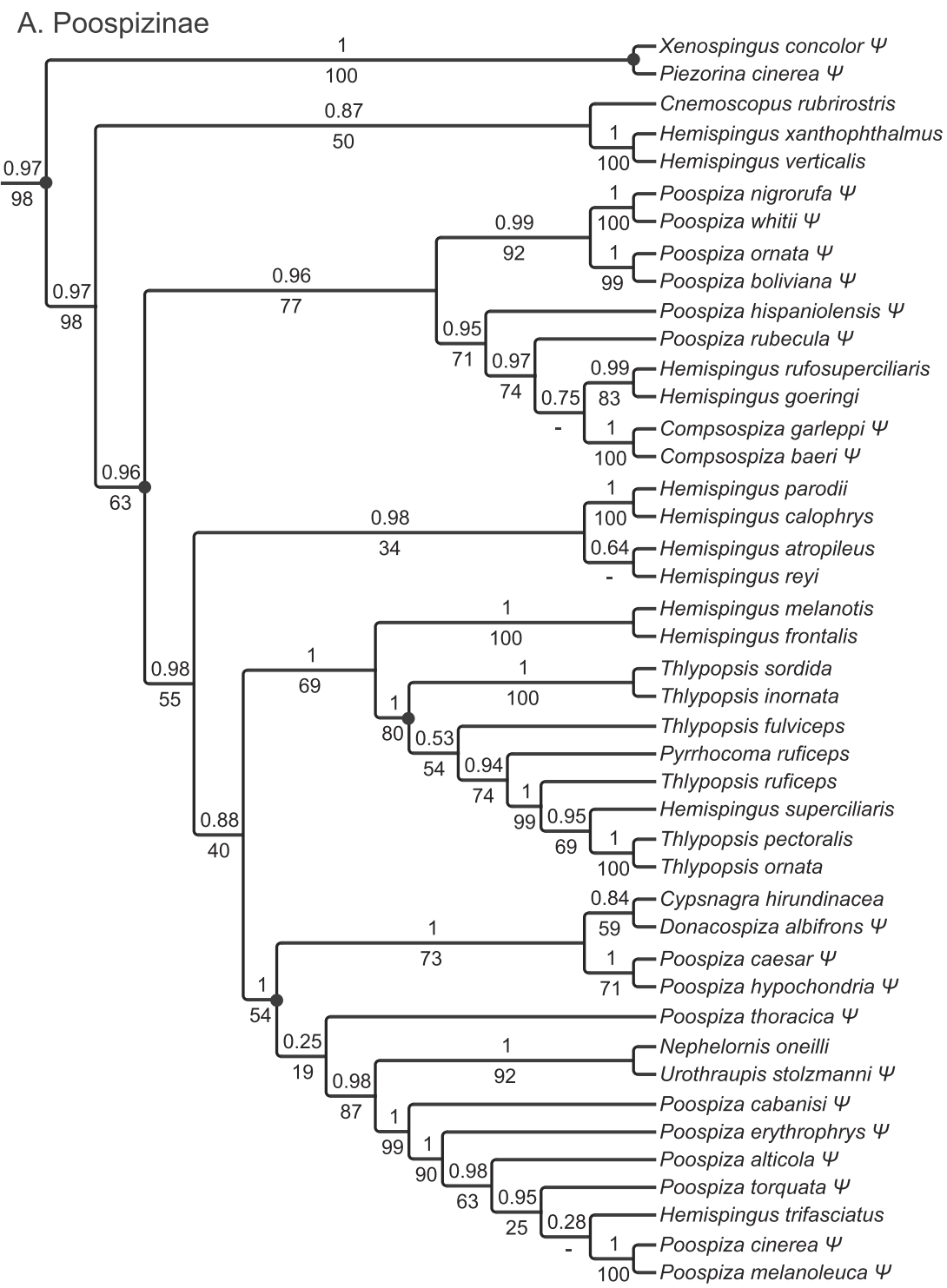

Fig. 4. Results of the concatenated analysis of the six genes for the subfamily Poospizinae. Labeling and format as in Fig. 2.

2014). The similarities between the results of our subfamily model-fitting and BAMM analyses are an excellent example of the first point; Sporophilinae was found to operate under a different diversification process by both approaches. With respect to Coerebinae, model-fit comparison found constant-rate diversification to fit best, but BAMM showed that it is actually a subclade within Coerebinae that is undergoing a different diversification process. The ability to detect different processes within a larger phylogeny was already possible with constant-rate diversification (Alfaro et al., 2009), but BAMM allows for the inclusion of time-varying speciation rates (Rabosky and Matute, 2013; Rabosky, 2014).

\subsection{Phylogenetic conclusions and taxonomic recommendations}

In this section, we indicate how our phylogenies compare to previous studies of tanager relationships and highlight places where our phylogenies shed light on the evolution of specific characters. Because of the large size of the tanager family, we organize the discussion of these conclusions around a proposed subfamily classification of tanagers. Such a classification is warranted in order to reasonably manage the large size of the group. These subfamilies were identified based on the oldest, strongly supported nodes in our phylogenies (Fig. 1). For each subfamily, we assign the family-group name that was used first for any species in the clade. When no previous name is available, a new name is proposed and a description provided (Appendix A). In one case (Poospizinae), a name was previously used (Wolters, 1975-1982), but a description was not provided. Thus, we provide a description here. Because the relationships among them are unknown, we generally present these subfamilies in order from least to most speciose. Relatively few avian groups have been sampled as comprehensively as the tanagers, and our study revealed the lack of concordance between currently used genus names and phylogeny. Thus, we predict that major generic revisions will be required in other avian groups when they are sampled at the species level. Although a new genus-level classification of tanagers is necessary, proposing new generic names is beyond the scope of this paper but is forthcoming. However, where possible, we propose the use of existing generic names that can reconcile classification with the topologies found in our study.

\subsubsection{Catamblyrhynchinae, Ridgway 1901, the Plushcap}

This subfamily contains a single species, the Plushcap (Catamblyrhynchus diadema), named for its unusual dense, velvety patch 
A. Diglossinae

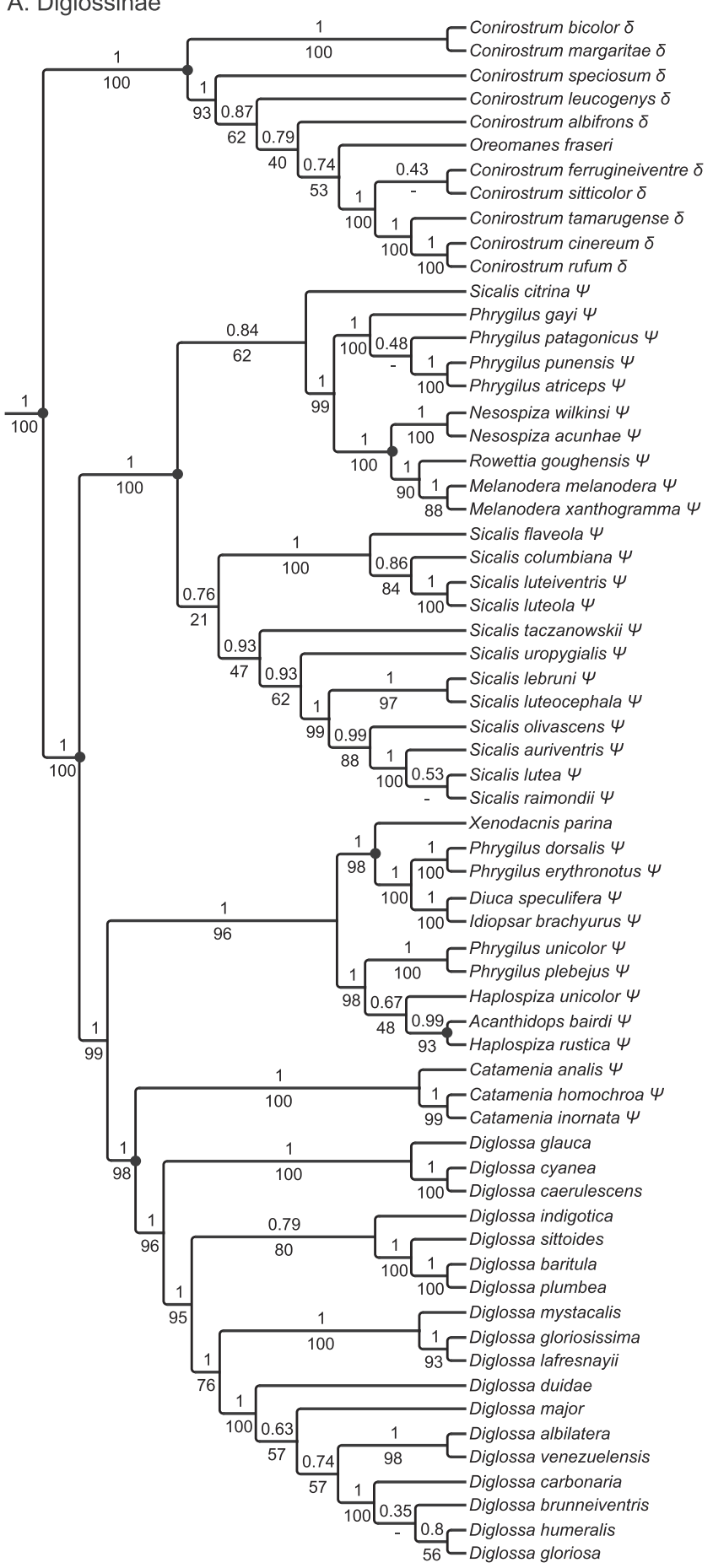

Fig. 5. Results of the concatenated analysis of the six genes for the subfamily Diglossinae. Labeling and format as in Fig. 2.

of bright yellow feathers on its forecrown. Hilty et al. (1979) speculated that these short, dense feathers are less susceptible to feather wear and more resistant to moisture than typical feathers. This may be an adaptation for its specialized feeding mode, in which it uses its thick, stubby bill to probe into dense whorls of Chusquea bamboo for its prey items (Hilty et al., 1979). This bill shape is convergent with other bamboo-feeding species in the Old World Paradoxornis (Olson, 1986). Because of its unique morphology and behavior, the taxonomic position of the Plushcap has eluded systematists for decades. Sharpe (1888) included it as one of many genera in his large family Fringillidae, which contained other New
World sparrows and finches, grosbeaks, buntings, and true finches. Subsequently, Ridgway (1901a) elevated the species to its own family, Catamblyrhynchidae. Later authors continued to consider the Plushcap to represent a monotypic family (e.g., Hellmayr, 1938; Meyer de Schauensee, 1966) or subfamily (Catamblyrhynchinae; e.g., Paynter and Storer, 1970; Ridgely and Tudor, 1989). More recent taxonomies have classified the Plushcap as a species of tanager (e.g., Sibley and Monroe, 1990; Clements et al., 2013), partly based on DNA hybridization studies that show it to be allied to the tanagers (Bledsoe, 1988). The placement of the Plushcap within the tanagers was also indicated by mtDNA sequence data (Yuri and Mindell, 2002) and multi-locus DNA data (Barker et al., 2013). In the present study, we confirm that the Plushcap is a tanager and is distinct from all other tanagers. Although the species was found to be sister to Porphyrospizinae in our BEAST and ML trees (Fig. 1), support was so low (0.74 PP; 26\% bootstrap) that this branch should not be treated with any special significance. Furthermore, none of the individual gene trees agree in their placement of Catamblyrhynchus, and none provide strong support for a relationship between Catamblyrhynchus and other tanager species (Supplementary Figs. 1-6). Thus, C. diadema is best thought of as a distinct tanager lineage, and we therefore place it in its own subfamily.

\subsubsection{Charitospizinae, new subfamily; the Coal-crested Finch}

Similar to the Plushcap, our analyses also identified the Coalcrested Finch (Charitospiza eucosma) as a distinct lineage with no closely allied extant relatives. This species is endemic to the grasslands of Brazil, has an unusual bimodal breeding season (Diniz et al., 2013), and appears to be a fire-following specialist (Cavalcanti and Alves, 1997; Jaramillo, 2011a). Its plumage colors and patterns are not like those of other tanagers, and both sexes possess a crest, a relatively rare feature in tanagers. Unlike the Plushcap, the Coal-crested Finch has not been previously recognized as distinct above the genus level. Early taxonomies (e.g., Sharpe, 1888) classified it in the same genus as other finches, with Oberholser (1905) being the first to place this species in its own genus. Miller (1928) indicated that Charitospiza might be closely related to Lophospingus based on similarities in their crests; however, he still argued that other features justified treating Charitospiza as generically distinct. Subsequent taxonomies continued to recognize this species as belonging to its own genus (e.g., Clements et al., 2013; Dickinson, 2003; Hellmayr, 1938; Paynter and Storer, 1970; Sibley and Monroe, 1990) but generally considered it to be more closely allied to finches than tanagers. Barker et al.'s (2013) genus-level study showed that this species is a tanager, and our phylogenies confirm this finding. In addition, we show that the Coal-crested Finch is distinct from all other tanagers, and is not closely related to any one species or group of tanager. In particular, there is no evidence for a close relationship between Lophospingus and Charitospiza as originally suggested by Miller (1928). Thus, the crests of these two species must have evolved convergently. Although our BEAST and ML trees show Charitospiza as the sister taxon to a large clade of other tanagers (Fig. 1), the support for this relationship is relatively low ( $0.85 \mathrm{PP} ; 23 \%$ bootstrap). Furthermore, none of the individual gene trees showed significant support for the placement of Charitospiza (Supplementary Figs. 1-6); therefore, we place $C$. eucosma in its own subfamily. This species is likely a relatively old lineage that is as evolutionarily distinct as some larger tanager clades with dozens of species. The Coal-crested Finch is listed as near-threatened due to habitat loss and trapping for the caged-bird trade (Birdlife International, 2013a). Given the importance of preserving evolutionarily distinct taxa, our finding that Charitospiza represents an old, distinct lineage argues for prioritizing its conservation. 

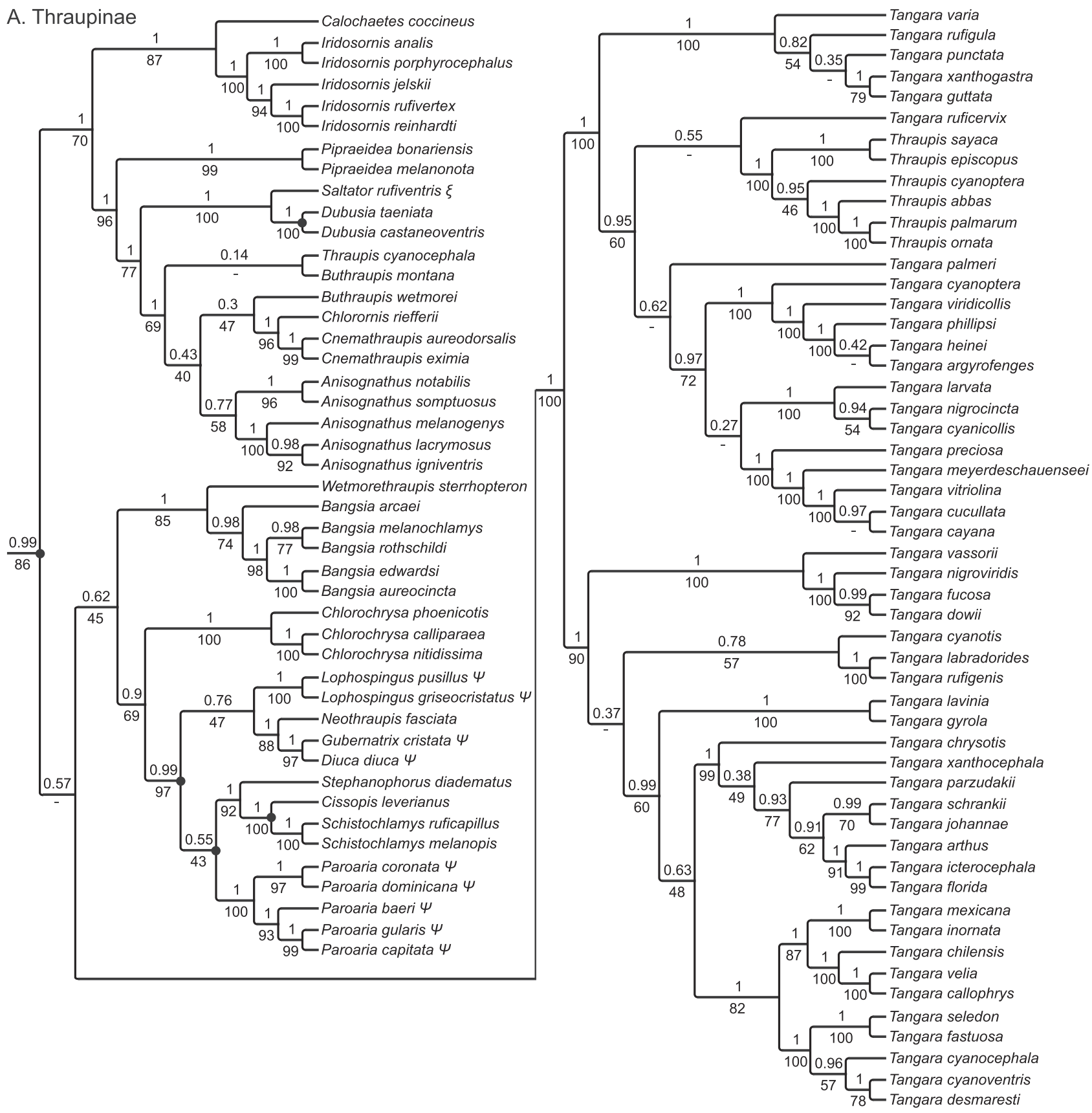

Fig. 6. Results of the concatenated analysis of the six genes for the subfamily Thraupinae. Labeling and format as in Fig. 2.

\subsubsection{Orchesticinae, new subfamily; the Grosbeak Tanagers}

This subfamily consists of just two species each in its own genus, the Yellow-shouldered Grosbeak (Parkerthraustes humeralis) and the Brown Tanager (Orchesticus abeillei). Due to the comparatively thick bills of these species, we refer to them as the grosbeak tanagers. Taxonomists have puzzled over the evolutionary affinities of each of these species, and thus they were placed in monotypic genera. $P$. humeralis was originally described as closely related to some species of saltators, and has subsequently been considered closely related to either saltators (e.g., Chapman, 1926) or cardinal-grosbeaks in Caryothraustes (e.g., Hellmayr, 1938; Paynter and Storer, 1970; Ridgway, 1901a; Sibley and Monroe, 1990). However, an allozyme study by Demastes and Remsen (1994) showed that it is not closely related to either saltators or grosbeaks; therefore, Remsen (1997) removed the species from Caryothraustes and proposed Parkerthraustes as a new genus. This recommendation was followed in subsequent taxonomic treatments (Dickinson, 2003; Clements et al., 2013). Klicka et al. (2007) analyzed cyt $b$ and ND2 sequences of a variety of tanagers and cardinal-grosbeaks and showed that Parkerthraustes belonged with tanagers, not cardinal-grosbeaks. However, Klicka et al. (2007) were not able to identify the closest relative of Parkerthraustes within the tanagers. In agreement with Barker et al. (2013), our data show that Parkerthraustes forms a strongly supported (1.0 PP; $100 \%$ bootstrap) clade with the Brown Tanager, 0 . abeillei (Figs. 1 and $2 \mathrm{c}$ ). This species is endemic to southeastern Brazil and shares little with Parkerthraustes in terms of plumage coloration and pattern. However, the Brown Tanager occurs in close association with Philydor rufum (the Buff-fronted Foliage-gleaner), and several studies have provided evidence that the Brown Tanager is a social mimic of this species (Beauchamp and Goodale, 2011; Sazima, 2010; Willis, 1976, 1989). Philydor rufum and O. abeillei are remarkably 
Table 3

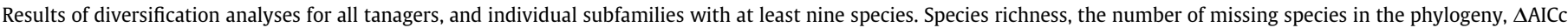

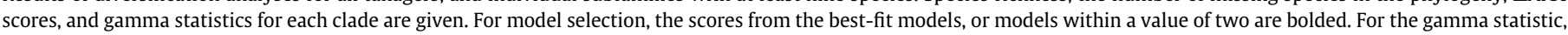
all significant $(P<0.05)$ tests are bolded.

\begin{tabular}{|c|c|c|c|c|c|c|c|c|c|}
\hline \multirow[t]{2}{*}{ Clade } & \multirow[t]{2}{*}{ Species Richness } & \multirow[t]{2}{*}{ \# Missing Species } & \multicolumn{5}{|c|}{ Diversification Model Comparisons ( $\Delta$ AICc) } & \multicolumn{2}{|c|}{ Gamma Statistic } \\
\hline & & & $P B$ & $B D$ & $D D L-E$ & $D D X$ & $D D L+E$ & Gamma & MCCR p-value \\
\hline Thraupidae (All Tanagers) & 371 & 16 & 47.24 & 52.24 & 22.97 & $\mathbf{0}$ & $\mathrm{N} / \mathrm{A}$ & $-\mathbf{5 . 6 1 1}$ & 0.001 \\
\hline \multicolumn{10}{|l|}{ Subfamily } \\
\hline Thraupinae & 102 & 4 & 39.02 & 41.11 & 14.73 & 15.23 & $\mathbf{0 . 0 0}$ & -4.845 & 0.001 \\
\hline Diglossinae & 64 & 0 & 4.21 & 6.35 & 1.95 & 0.00 & 180.90 & -1.850 & 0.029 \\
\hline Poospizinae & 44 & 1 & 7.72 & 9.93 & 0.00 & 2.08 & 307.80 & -2.839 & 0.003 \\
\hline Sporophilinae & 38 & 5 & 0.00 & 1.26 & 2.28 & 2.28 & 405.67 & 0.783 & 0.831 \\
\hline Tachyphoninae & 31 & 1 & 4.31 & 6.62 & 2.24 & 0.00 & 368.66 & -1.740 & 0.043 \\
\hline Coerebinae & 29 & 0 & 0.00 & 0.05 & 2.33 & 2.20 & 407.61 & 1.222 & 0.897 \\
\hline Saltatorinae & 16 & 0 & 4.34 & 7.03 & 0.00 & 4.61 & 462.95 & -2.092 & 0.025 \\
\hline Dacninae & 14 & 1 & 2.66 & 5.59 & 0.00 & 2.14 & 478.95 & -1.782 & 0.040 \\
\hline Hemithraupinae & 9 & 0 & 0.00 & 3.73 & 1.96 & 1.16 & 502.37 & -0.646 & 0.272 \\
\hline Porphyrospizinae & 9 & 2 & 2.57 & 7.57 & 0.00 & 4.56 & 524.41 & -1.560 & 0.072 \\
\hline
\end{tabular}

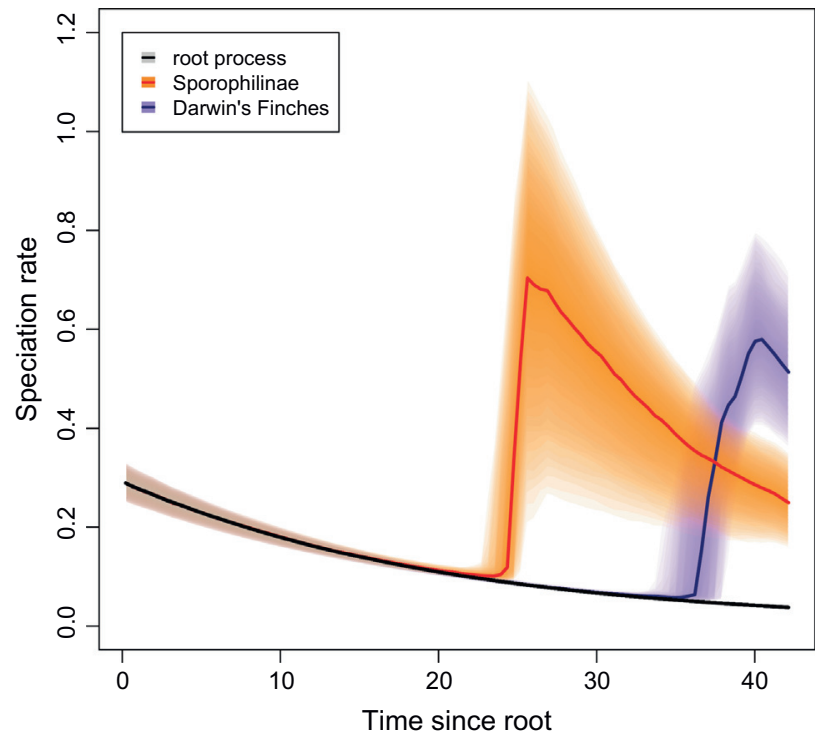

Fig. 7. Diversification models for tanagers. The three diversification processes that describe tanager diversification are plotted separately. The root process is described by a model that governs the entire phylogeny, excluding the Sporophilinae and Coerebinae radiations. For these radiations, the model describing the root process was used to define the speciation rate until the transition to a new diversification model. Results were averaged from 1000 samples from the BAMM posterior distribution of results. The shaded regions represent $25-75 \%$ confidence intervals.

similar in plumage; both have overall cinnamon brown plumage, rufous wings, a dark eye line, and a midcrown and nape that are darker than the rest of the head, among other similarities. The two species flock together, with $P$. rufum more common than $O$. abeillei; thus, Willis (1989) hypothesized that 0 . abeillei evolved similar plumage as a means of predator avoidance. The close evolutionary relationship of Orchesticus to the differently plumaged Parkerthraustes supports this social mimicry hypothesis. Although different in plumage coloration, Parkerthraustes and Orchesticus are generally similar in overall size, form, bill size, and bill shape. Strong selection acting on plumage color in Orchesticus could have masked the shared evolutionary history of Parkerthraustes and Orchesticus.

\subsubsection{Nemosiinae, Bonaparte, 1854; the Flock-dwelling Tanagers}

This small subfamily consists of only five species divided among four genera: three are monotypic (Cyanicterus, Sericossypha, Compsothraupis) and one (Nemosia) has two species. We sampled all but one ( $N$. rourei) of these five species. Species in this group are some of the most spectacular of all tanagers, and these species have some of the most sexually dichromatic plumages (Burns and Shultz, 2012). The group is made up of seemingly disparate taxa whose relationships have puzzled ornithologists for decades. The lack of understanding of their relationships is reflected in the proportion of monotypic genera in the group; however, there are some common plumage themes. Three species (Sericossypha albocristata, Compsothraupis loricata, and $N$. rourei) have red throats, four species (S. albocristata, Cyanicterus cyanicterus, N. rourei, and N. pileata) have bluish plumage, and $S$. albocristata and some individuals of $N$. rourei have white crowns. In addition, most of the species often occur in single-species flocks (Isler and Isler, 1999; Venturini et al., 2005). Prior to molecular studies, a close relationship among all these species was not expected. Burns (1997) and Burns et al. (2003) included N. pileata and S. albocristata in phylogenetic analyses of cyt $b$ data for tanagers and recovered them as a clade, but support was not strong. Barker et al.'s (2013) genus-level phylogeny found strong support for the monophyly of this group, and our concatenated analyses (Figs. 1 and 2d) also recovered a strongly-supported Nemosiinae (1.0 PP; $100 \%$ bootstrap). Most of our individual gene analyses also recovered this clade, with RAG1, cyt $b$, and FGB-I5 all supporting it strongly (Supplementary Figs. 1-6).

S. albocristata and C. loricata, have been consistently placed next to each other in most classifications, with some authors (e.g., Meyer de Schauensee, 1966, 1970; Sick, 1993; Zimmer, 1947) considering them congeneric. These two species are among the largest tanagers, and both are more often found in flocks than in pairs. Otherwise, their appearance, behaviors, and geographic distributions are quite different; $S$. albocristata is an Andean species, whereas $C$. loricata is found in the Caatinga of northeastern Brazil. Thus, most authorities (e.g., Clements et al., 2013; Hellmayr, 1936; Sibley and Monroe, 1990) consider the differences between them significant enough to warrant generic separation. In addition, although they have been consistently placed within the tanagers, many authors have noted suspicions that one or both species might belong elsewhere. In particular, the resemblance between S. albocristata and the cotingas (e.g., Hellmayr, 1936; Sclater, 1886), and similarities between C. loricata and the blackbirds, have been frequently noted (e.g., Gwynne et al., 2010; Ridgely and Tudor, 1989; Zimmer, 1947). Suspicions regarding a connection between $S$. albocristata and the cotingas were largely put to rest by Morony's (1985) study of skeletal anatomy. He established the placement of $S$. albocristata within the nine-primaried oscines, and later molecular studies (Burns, 1997; Burns et al., 2003) cemented its position within Thraupidae. However, the phylogenetic position of $C$. loricata has remained enigmatic. We were able to se- 
quence cyt $b$ from a toe pad of this species and our results show a strongly supported sister relationship between $C$. loricata and $S$. albocristata. Thus, despite some authors' expectation that any similarities between the two taxa might be the result of convergence (e.g., Storer, 1970), and suspicions that $C$. loricata might be a blackbird, our study shows that they are closely related tanagers.

Although the relationship between S. albocristata and C. loricata is well supported (1.0 PP; $100 \%$ bootstrap), relationships among the other species in this subfamily are unclear from our analyses (Fig. 2d, Supplementary Figs. 1-6). Other than Barker et al. (2013), no previous study has addressed relationships of Cyanicterus cyanicterus using molecular data. This unusual tanager from northern South America is mostly blue and yellow and has a relatively thick, slightly curved bill. Early classifications (Hellmayr, 1935; Sclater, 1886) placed this species near Piranga, which is now known to belong to Cardinalidae (Klicka et al., 2007). Later, Storer (1970) moved it to a position adjacent to Buthraupis due to similarities in color, pattern, and plumage texture. Subsequent classifications (e.g., Sibley and Monroe, 1990) followed this arrangement. Our molecular data place C. cyanicterus firmly in Nemosiinae; however, understanding the relationship of Cyanicterus within Nemosiinae will require additional data.

The remaining genus in this subfamily, Nemosia, consists of two species: $N$. pileata and the recently rediscovered $N$. rourei. The phylogenetic relationship of Nemosia to other tanagers has been unclear. Previous classifications have considered species in presentday Nemosia to be closely related to Hemithraupis, Pyrrhocoma, and Chrysothlypis (Hellmayr, 1936; Sclater, 1886; Sibley and Monroe, 1990; Storer, 1970), with some classifications (e.g., Sclater, 1886) considering Hemithraupis and Nemosia congeneric. However, our phylogenies show that species in these genera are all part of other subfamilies and not closely related to Nemosia. We were able to include one Nemosia species (N. pileata) in our analyses and we confirmed earlier findings based on cyt $b$ and partial taxon sampling (Barker et al., 2013; Burns, 1997; Burns et al., 2003) that Nemosia is part of Nemosiinae. Although our ML analyses recovered a strongly supported (100\% bootstrap) sister relationship between Nemosia and the clade containing Compsothraupis and Sericossypha, this relationship was not recovered in BEAST analyses and was not consistently recovered in single gene analyses (Fig. $2 \mathrm{~d}$, Supplementary Figs. 1-6). Thus, with the exception of the sister relationship between Compsothraupis and Sericossypha, relationships of other species within Nemosiinae remain unclear.

We were unable to include samples of the extremely rare and recently re-discovered N. rourei (Bauer et al., 2000; Scott, 1997). However, this species shares similarities with other species in Nemosiinae that lead us to conclude that it belongs in this clade as well. Eye color, plumage colors, and plumage patterns are similar between $N$. rourei and $N$. pileata. Also, S. cristata, C. loricata, and $N$. rourei have red throats, and the crowns of some individuals of $N$. rourei are white like those of S. cristata (as illustrated in Venturini et al., 2005). Venturini et al. (2005) also note apparent similarities in courtship between $N$. pileata and $N$. rourei. Overall, pending further analyses, we recommend retaining $N$. rourei in Nemosia.

\subsubsection{Hemithraupinae, Sundevall, 1872; the Yellow-and-black}

\section{Tanagers}

This subfamily consists of nine species of mostly slender-billed tanagers placed in five different genera. We sampled all nine species, and our phylogeny is consistent with current genus-level classifications. With the exception of the two species of Heterospingus, species in this subfamily have some of the thinnest bills in relation to bill length of all tanagers. In addition, species in this subfamily are some of the most sexually dichromatic of all tanagers (Burns and Shultz, 2012). Yellow and black are predominant plumage colors in the group, although males of Chrysothlypis salmoni are bright red, and males of Chlorophanes spiza are mostly blue. In addition, most species (those in Chlorophanes, Iridophanes, and Hemithraupis) have dark upper mandibles and yellow lower mandibles. The node uniting Hemithraupinae was strongly supported in our concatenated analyses (Fig. 2g; 1.0 PP; 99\% bootstrap), and this clade was also recovered in the genus-level study of Barker et al. (2013). The clade was recovered, though not strongly supported, in earlier analyses that included only cyt $b$ and partial taxon sampling (Burns et al., 2003). Previous workers had not suspected a close relationship among all the species in this clade, but some had predicted a close relationship between some of the genera.

Iridophanes pulcherrimus and Chlorophanes spiza were previously classified in a family of nectar-feeding honeycreepers (Hellmayr, 1935; Sclater, 1886) that subsequently was shown to be polyphyletic (Burns et al., 2003). These two species form a clade that is sister to the other members of this subfamily. This clade has strong support in our concatenated analyses (1.0 PP: 100\% bootstrap), is strongly supported in all but one of our gene trees, and was also supported in the genus-level analyses of Barker et al. (2013). Ridgway (1901b) erected Iridophanes, and considered it closely related to other species in our new subfamily such as Chrysothlypis chrysomelas and species in Hemithraupis (Ridgway, 1902). Among other earlier workers, only Hellmayr (1935) indicated that Chlorophanes and Iridophanes were likely closely related. He noted similarities in the bill structure (enlarged basal portion of the lower bill) and bill color (yellowish margin of the cutting edge of the lower bill) of both species. Subsequent to Hellmayr (1935), the remarkable similarity in plumage pattern between Iridophanes and Tangara cyanoptera led Storer (1970) to merge Iridophanes into Tangara. However, others (e.g., Ridgely and Tudor, 1989) were not comfortable with this merger, noting differences in eye color, bill shape, and bill color; these are characters that Iridophanes shares with Chlorophanes. After the study of Burns et al. (2003), Chlorophanes and Iridophanes were typically considered closely related (e.g., Clements et al., 2013; Dickinson, 2003), with Hilty (2011) suggesting the merger of both species into Chlorophanes. We suggest keeping the two species in separate genera, mainly to promote taxonomic stability. In addition, our data indicate that each species has been evolving separately for a relatively long period of time (Supplementary Figs. 1-6).

For the remaining three genera in this subfamily (Heterospingus, Hemithraupis, and Chrysothlypis), a close relationship among some of the species was implied in the classifications of earlier workers. Ridgway (1902) classified species from all three genera near each other, Sclater (1886) classified species of Heterospingus and Chrysothlypis adjacently, and other classifications (Hellmayr, 1936; Sibley and Monroe, 1990) placed Hemithraupis and Chrysothlypis together. Our phylogenies find strong support (1.0 PP; $98 \%$ bootstrap) for a monophyletic group containing all the species in these genera. Our concatenated trees show that Hemithraupis is more closely related to Heterospingus than it is to Chrysothlypis; however, this node was not strongly supported ( $0.84 \mathrm{PP} ; 58 \%$ bootstrap). Ridgely and Tudor $(1989,2009)$ suggest that Hemithraupis and Chrysothlypis could be merged into a single genus. However, merging them into a genus without Heterospingus is not supported by our topologies.

Chrysothlypis contains two species, C. salmoni and C. chrysomelas. Females of these species have similar plumage and are mostly yellow, like other species in this subfamily. However, males of the two species are quite different: $C$. salmoni males are bright red with white bellies, whereas $C$. chrysomelas males are yellow and black. Thus, early classifications separated the two species, with C. chrysomelas often placed in the monotypic Erythrothlypis (e.g., Hellmayr, 1936). Storer (1970) was the first to merge these two species 
into the same genus, and subsequent workers have largely followed this placement. More recently, several authors (Hilty, 2011; Ridgely and Greenfield, 2001; Ridgely and Tudor, 2009) have returned to using Erythrothlypis for C. chrysomelas. Our results show that the two species are not highly divergent (mtDNA uncorrected sequence divergence $=5.6 \%$ ). In addition, despite radically different male plumages, the two species are similar in female plumage and overall size and shape. Therefore, we advocate the continued use of Chrysothlypis for both of these species.

Within Hemithraupis, H. guira and H. ruficapilla are regarded as a superspecies (Sibley and Monroe, 1990) and are known to hybridize in southeastern Brazil (Hilty, 2011; Ridgely and Tudor, 1989). We report the first genetic data for $H$. ruficapilla and show that the two species are more closely related to each other than they are to $H$. flavicollis. Levels of genetic divergence between $H$. guira and $H$. ruficapilla are relatively low (cyt $b$ uncorrected difference $=1.2 \%$, but consistent with species-level status for these two taxa. Heterospingus rubrifrons and H. xanthopygius are also regarded as a superspecies (Sibley and Monroe, 1990), with some classifications considering them conspecific (Storer, 1970; Isler and Isler, 1999). We found that the two species are sister taxa and well differentiated genetically. Uncorrected cyt $b$ sequence difference between the two individuals is $2.8 \%$ and ND2 pairwise difference is $5.6 \%$; these values are consistent with many other well-differentiated species of tanagers.

\subsubsection{Porphyrospizinae, new subfamily; the Yellow-billed Tanagers}

This clade consists of nine species; most of these are in genera traditionally considered part of Emberizidae (Incaspiza, 5 species; Phrygilus, 3 species), and one (Porphyrospiza, 1 species) was traditionally placed in Cardinalidae (Fig. 2f). Before Barker et al. (2013), no study had suggested a close relationship among all these species; however, species in this group share some plumage, behavioral, and morphological characters, including yellow-colored bills. Our concatenated phylogeny supports their monophyly (0.94 PP; 99\% bootstrap), and three of our gene trees also provide strong support (cyt $b$, ND2, and RAG1).

Within this subfamily, Incaspiza consists of five Peruvian endemics that prefer arid scrub habitats. We sampled three of the species and found strong support for their monophyly (1.0 PP; $100 \%$ bootstrap). Species status of these has been questioned in previous taxonomies. Both Hellmayr (1938) and Paynter and Storer (1970) considered I. pulchra and I. personata as conspecific. When Zimmer (1952) described I. ortizi, he indicated the possibility that I. ortizi and I. pulchra might be conspecific, although he argued that there was more evidence that they were separate species. Later, Paynter and Storer (1970) also suggested that I. ortizi might also be conspecific with I. pulchra and I. personata. Sibley and Monroe (1990) treated the three forms as separate species of the same superspecies. Our data support species status for each. Despite similarities in plumage, the three are genetically very distinct, with uncorrected cyt $b$ divergence ranging from $7.5 \%$ to $9.6 \%$, well above typical values observed within avian species. Ridgely and Tudor (1989) place I. pulchra, I. personata, and I. ortizi in the same group, which they named group $A$, and the other two species (I. laeta and I. watkinsi) in a separate group B. Because we were unable to sample members of group B, we cannot confirm the monophyly of these two groups. We found strong support (1.0 PP; $99 \%$ bootstrap) for a closer relationship between I. ortizi and I. personata than between either of these and I. pulchra. The additional species within Incaspiza need to be sampled before further conclusions can be made about evolution within this group, but we have no reason to suspect that the two species missing from our data set belong outside Incaspiza.

Incaspiza is sister to a clade containing the monotypic Porphyrospiza and three of the 11 species of Phrygilus (P. alaudinus,
P. carbonaria, and P. fruticeti). Phrygilus is one of the most polytypic tanager genera (Campagna et al., 2011). Although three species belong in Porphyrospizinae, the remaining eight species belong to three distinct clades in the Diglossinae (Section 4.3.14). Porphyrospiza was considered a cardinal-grosbeak by Hellmayr (1938); however, based on skull osteology, Tordoff (1954) argued that this species was more closely allied to sparrows that we now consider tanagers. However, Paynter and Storer (1970) disagreed with Tordoff (1954) and continued to place Porphyrospiza with the cardinalgrosbeaks, a position followed by many authors (e.g., Sibley and Monroe, 1990). Furthermore, citing plumage similarities between Porphyrospiza and Passerina buntings (e.g., Allen, 1891), Paynter and Storer (1970) merged Porphyrospiza with Passerina, a genus of cardinal-grosbeaks. Other authors have argued that these similarities are superficial (Bates et al., 1992; Ridgely and Tudor, 1989), and thus other taxonomies (e.g., Clements et al., 2013; Dickinson, 2003) have removed them from the cardinal-grosbeaks. DNA evidence (Barker et al., 2013; Klicka et al., 2007) clearly shows that Porphyrospiza belongs with the tanagers. Our study found that Porphyrospiza is embedded within a clade of the three yellow-billed sierra finches (Phrygilus alaudinus, P. carbonaria, and P. fruticeti). These four species have yellow bills and similar, streaked females. Based on morphology, other authors have indicated a close relationship of these three Phrygilus (e.g., Fjeldså, 1992; Ridgely and Tudor, 1989). Using molecular data, Klicka et al. (2007) showed a close relationship between Porphyrospiza and P. alaudinus, Barker et al. (2013) showed a close relationship between Porphyrospiza and P. fruticeti, and Campagna et al. (2011) found a monophyletic clade containing the three Phrygilus. Here, by sampling all species, we found that the three yellow-billed Phrygilus are not monophyletic. Instead, our concatenated tree had strong support (0.99 PP; $100 \%$ bootstrap) for a clade containing Porphyrospiza and two of these Phrygilus ( $P$. alaudinus and $P$. carbonarius), with $P$. fruticeti as the sister taxon to the clade containing these three species. Because Phrygilus is polyphyletic, and the type species of Phrygilus ( $P$. gayi) belongs in Diglossinae, a new generic taxonomy for these species is necessary. For $P$. fruticeti, we recommend using the available name Rhopospina Cabanis 1851 (type $=P$. fruticeti). For $P$. alaudinus and $P$. carbonarius, the name Corydospiza Sundevall 1872 (type $=P$. alaudinus $)$ is available.

\subsubsection{Dacninae, Sundevall, 1836; the Blue Tanagers}

This relatively small clade contains 14 species in three monophyletic genera, Cyanerpes, Dacnis, and Tersina. Of all the subfamilies, Dacninae is the most sexually dichromatic (Burns and Shultz, 2012), with species characterized by a theme of bright blue males and green females. In addition, species in this subfamily have some of the most ultraviolet-reflecting plumage of all tanagers (Burns and Shultz, 2012). Plumage colors of these species are similar not only from a human visual perspective, but also in the shape of their reflectance curves (Barreira et al., 2008; Burns and Shultz, 2012). In contrast to these plumage similarities, species in this clade have dramatic differences in bill shapes and foraging behavior. The Swallow-Tanager, the only species in Tersina, has many unique behavioral and morphological characters including a flattened bill that is broad at its base (Isler and Isler, 1999; Lucas, 1895; Schaefer, 1953; Webster, 1988). These characteristics have led the SwallowTanager to be described in classifications as a monotypic family (Hellmayr, 1936; Meyer de Schauensee, 1970; Wetmore, 1960), subfamily (Sclater, 1886; Storer, 1970), or tribe (American Ornithologists' Union, 1983). However, subsequent DNA data have confirmed the placement of Tersina well within the tanagers (Sibley and Ahlquist, 1990), and more specifically, closely related to species in Dacnis and Cyanerpes (Burns et al., 2003). Although species in all three genera will feed on fruit and insects, differences in diet are reflected by differences in bill shape. The Swallow-Tanager 
often sallies from exposed perches, using its unique bill to capture flying insects (Hilty, 2011; Isler and Isler, 1999; Restall et al., 2006). Unlike the Swallow-Tanager, species in Dacnis and Cyanerpes include nectar in their diet. Species in Cyanerpes are nectarivorous (Ridgely and Tudor, 2009), and have narrow, long, decurved bills. Many species of Dacnis also feed on nectar (Hilty, 2011). Like species in Cyanerpes, the bills of Dacnis are also narrow; however, they are overall shorter and more pointed, and the behavior of some species of Dacnis is more warbler-like (Restall et al., 2006; Ridgely and Tudor, 2009). This diversity of bill types, which evolved over a relatively short amount of time, highlights one of the major themes of tanager phylogeny: the lability of bill morphology and foraging behavior. The monophyly of this subfamily is strongly supported by our concatenated analyses (1.0 PP; $100 \%$ bootstrap) and the topologies of three individual gene trees (Cyt $b$, ND2, and RAG1) (Figs. 1 and 2h; Supplementary Figs. 1-6).

Our phylogenies also clearly identify that each of these three genera is monophyletic; therefore, no changes to the genus-level taxonomy are necessary. However, we did not find strong support for the placement of the three genera with respect to each other. Species-level relationships within Cyanerpes and Dacnis have not been addressed by previous studies using molecular data. Within Cyanerpes, we found two strongly supported clades, one with $C$. caeruleus and $C$. cyaneus and the other with $C$. nitidus and $C$. lucidus (Fig. 2h). This relationship contrasts with the idea that $C$. lucidus and C. caeruleus form a superspecies (Sibley and Monroe, 1990) or that they are conspecific (Hellmayr, 1935). Within Dacnis, the only strongly supported nodes identify a sister relationships between $D$. venusta and the remaining species. D. hartlaubi was originally described by Sclater (1854) as a member of Dacnis, but it was subsequently thought to be more similar to species in Tangara. Unsure of its relationships, Sclater (1886) placed it in its own monotypic genus, Pseudodacnis, which was subsequently used by many authors (e.g., Hellmayr, 1936; Meyer de Schauensee, 1966, 1970). Although Storer (1970) merged Pseudodacnis with Dacnis based on similar coloration and pattern, not all classifications have followed this recommendation (e.g., Sibley and Monroe, 1990). The results of our study show that this species is embedded well within Dacnis, with strong support; therefore, the use of Pseudodacnis is no longer warranted. We were not able to sample one species of Dacnis, $D$. berlepschi. This species is quite different from other species in the genus, and we regard its placement as uncertain. Superficially its plumage resembles that of Xenodacnis parina, which we place in Diglossinae (Section 4.3.14). Storer (1970) also doubted whether $D$. berlepschi was closely related to other species of Dacnis, and Wolters (1975-1982) also considered X. parina and D. berlepschi closely related, placing both within the genus Xenodacnis.

\subsubsection{Saltatorinae, Bonaparte, 1853; the Saltators}

We found a strongly supported clade (1.0 PP; 79\% bootstrap) that contained 15 of the 16 species of Saltator as well as the sole member of Saltatricula (S. multicolor) (Figs. 1 and 2i). Saltators have relatively long tails, well developed hindlimbs, and some of the thickest bills of all tanagers. The only species in Saltator not found in this clade is S. rufiventris, which belongs in Thraupinae (Section 4.3.15). Unlike many subfamilies of tanagers, all nodes within Saltatorinae were strongly supported in either BEAST, ML or both analyses (Fig. 2i). Saltators have long been considered part of Cardinalidae (cardinal-grosbeaks) and, although some workers (e.g., Sushkin, 1924) have suggested they are tanagers, recent classifications have continued to place the saltators with the cardinal-grosbeaks (e.g., Dickinson, 2003; Orenstein, 2011; Sibley and Monroe, 1990). Despite this practice, recent molecular analyses (Barker et al., 2013; Klicka et al., 2007) show they belong with tanagers. Although Klicka et al. (2007) suggested that saltators might represent the sister taxon to the remaining tanagers, more complete character sampling and outgroup sampling (Barker et al., 2013), as well as more complete ingroup sampling (the present study), do not support this hypothesis. Instead, saltators are embedded within tanagers, sister to a clade containing the South American grassland finches and relatives (Emberizoidinae, Section 4.3.9; Fig. 1). Hellack and Schnell (1977) analyzed relationships among saltators using skeletal, external morphological, and color characters, but the species-level relationships of our phylogeny bear little resemblance to those of Hellack and Schnell (1977). In particular, Hellack and Schnell (1977) suggested that S. aurantiirostris, S. atricollis, and $S$. orenocensis may not belong with the rest of the saltators; however, all three fall within the saltator clade, and none are more closely related to each other than to other saltators. More recently, Klicka et al. (2007) included 11 species of saltators and sampled cyt $b$ and ND2. In our study, we included the remaining species and added nuclear genes. Our results agree with those of Klicka et al. (2007), with all the strongly supported nodes of Klicka et al. (2007) recovered in our phylogeny. Klicka et al. (2007) showed that the saltator clade also includes Saltatricula multicolor, the Many-colored Chaco Finch. This enigmatic taxon is usually classified in Emberizidae; however, other recent molecular phylogenies had also indicated that it is closely related to tanagers (e.g., Burns et al., 2003). Our results agree with these studies and show that it belongs with saltators and is sister to Saltator atricollis. Thus, we recommend merging Saltatricula with Saltator. Of all the species of Saltator, Saltatricula is most alike Saltator atricollis in plumage. The two species also share a preference for dry habitats and are both restricted to the Central South America zoogeographic region (Parker et al., 1996), with Saltatricula multicolor occurring in the dry scrub of the Chaco, and Saltator atricollis in the Caatinga and Cerrado scrub (Brewer, 2011; Jaramillo, 2011b; Parker et al., 1996). Both Hellack and Schnell (1977) and Ridgely and Tudor (2009) suggested that Saltator atricollis was atypical among saltators. The relationship of this species to Saltatricula and the sister relationship of the Saltatricula/Saltator atricollis clade to other saltators agrees with this assessment. Saltator grossus and S. fuliginosus were previously considered members of their own genus Pitylus (e.g., Ridgely and Tudor, 1989) on the basis of similar morphology and sexual dichromatism. In addition, the two have sometimes been considered members of the same species (Paynter and Storer, 1970) or part of a superspecies (Sibley and Monroe, 1990). Our data show a level of divergence expected between two species, and that the two species form a clade embedded within saltators. Thus, the two species are closely related, but the use of Pitylus is not warranted. Additional sets of saltator species have been grouped into subspecies or considered conspecific, and our data set provides genetic evidence to address these hypotheses. S. similis and S. coerulescens were thought to form a superspecies (Short, 1975; Sibley and Monroe, 1990), but are not sister taxa. Likewise, a superspecies composed of S. nigriceps, S. maxillosus, and S. aurantiirostris (Short, 1975; Sibley and Monroe, 1990) is not supported by our results. $S$. nigriceps and $S$. aurantiirostris were considered conspecific by Hellmayr (1938) and Paynter and Storer (1970), but are only distantly related in our phylogenies. The Lesser Antillean S. albicollis was long considered conspecific with continental S. striatipectus (Hellmayr, 1938; Paynter and Storer, 1970; Sibley and Monroe, 1990). Both species share similar, streaked plumage. Seutin et al. (1993) showed that populations of these two forms were well-differentiated by mtDNA restriction site variation. In our study, we confirm this finding using sequence data, further justifying their treatment as separate species.

Chaves et al. (2013) produced the most comprehensive molecular phylogeny of saltators to date. Compared to our study, Chaves et al. (2013) had more individuals per species sampled, but fewer genetic markers. The phylogenies of Chaves et al. (2013) are based on ND2 sequences from multiple individuals, as well as some 
additional cyt $b$ sequences from some species. In contrast, our study had mtDNA and nuclear DNA data from most species (Table 1 ), but we only sampled one individual per species. In general, the findings of the two studies are similar. For example, both studies identified the clade of Saltatricula multicolor and S. atricollis as sister to the rest of the saltators, and our study recovered the same three main clades (clades A, B, and C) of Chaves et al. (2013). However, Chaves et al. (2013) did not recover a sister taxon relationship between the two streaked saltators (S. albicollis and S. striatipectus). Instead, they found a sister taxon relationship between $S$. albicollis and $S$. similis and comparatively little genetic divergence between them. Additional study is needed to clarify the relationships among these species.

\subsubsection{Emberizoidinae, new subfamily, the Grassland Tanagers}

Some of our analyses support a sister relationship between Saltatorinae and Emberizoidinae, a clade containing six species classified into three different genera (Embernagra, Emberizoides, and Coryphaspiza). The Saltatorinae/Emberizoidinae clade is found in both our ML and Bayesian concatenated analyses; however, support was not particularly high ( $0.89 \mathrm{PP} ; 69 \%$ bootstrap). This clade was also recovered, but with weak support, in our ACO1-I9 and ND2 ML trees (Supplementary Figs. 1-6). Further study with additional data is needed to clarify whether the species in these two subfamilies are more closely related to each other than they are to other tanagers. Nevertheless, similar plumage colors and patterns of the two groups would support their close relationship.

Unlike saltators, which are mostly arboreal, the birds in Emberizoidinae are terrestrial, grassland dwelling birds. All six species were traditionally classified with the New World sparrows, but Sibley and Monroe (1990) considered them tanagers. The monophyly of Emberizoidinae is strongly supported by genus-level analyses (Barker et al., 2013), concatenated analyses (0.90 PP; 99\% bootstrap; Figs. 1 and $2 \mathrm{i}$ ), and separate ML analyses of individual genes (5 genes with greater than $70 \%$ support; Supplementary Figs. 1-6). Although the monophyly of this group is strongly supported, prior to Barker et al. (2013) no previous study has considered these species to form a unique clade. The Black-masked Finch (Coryphaspiza melanotis), the sole member of its genus, was originally described as a member of Emberizoides. The species was later placed in Coryphaspiza, and Gray (1870) considered Coryphaspiza a subgenus of Emberizoides. Sharpe (1888) came close to recognizing the unique relationship of the species in the grassland clade. He placed Embernagra, Coryphaspiza, and Emberizoides in order next to each other in his linear classification; however, he considered Donacospiza albifrons a member of Coryphaspiza. Later, Hellmayr (1938) considered D. albifrons a modified form of Poospiza, a result confirmed in our study (see Poospizinae, Section 4.3.13). Although he placed Coryphaspiza and Emberizoides adjacent to each other in his classification, he placed Embernagra elsewhere. In contrast, Paynter and Storer (1970) placed Embernagra and Emberizoides adjacent to each other, but put Coryphaspiza in another part of their classification, together with several other species they considered of uncertain placement. Subsequent classifications have mostly treated the species similarly, with Coryphaspiza and Embernagra/ Emberizoides placed apart from each other (e.g., Clements et al., 2013; Dickinson, 2003; Sibley and Monroe, 1990). However, Wolters (1975-1982) placed the three genera together in his linear classification, and Jaramillo (2011c) suggested that aspects of juvenile and adult plumage, tail shape, and song suggest that Coryphaspiza may be related to Embernagra and Emberizoides. Klicka et al. (2007) reported the first phylogeny that contained more than one of these genera and confirmed the close relationships of Emberizoides and Embernagra. The genus-level DNA study of Barker et al. (2013) identified that Coryphaspiza also belongs in this group. With greater character and species sampling, we confirm the monophyly of this novel clade of grassland birds. The identification of all these species as closely related should facilitate the future study of diversification in the South American grasslands. Our data can also address some of the taxonomic issues surrounding species limits in the group. The three species of Emberizoides were treated as conspecific for many years (Hellmayr, 1938, Paynter and Storer, 1970). However, Eisenmann and Short (1982) argued that E. ypiranganus and $E$. duidae are distinct from E. herbicola, and subsequent classifications have followed this recommendation (Clements et al., 2013; Sibley and Monroe, 1990). In addition, Sibley and Monroe (1990) treated E. herbicola and E. duidae as members of the same superspecies. We include all three species in our phylogeny and show levels of divergence consistent with separate species. We are missing one species of Embernagra, E. longicauda; however, we feel that this species shows enough similarities to the other Embernagra to retain it within this genus.

\subsubsection{Coerebinae, d'Orbigny and Lafresnaye, 1838; the Dome-nesting Tanagers}

This subfamily includes 29 species in 12 genera, all of which build covered or domed nests with side entrances (Burns et al., 2002). This is an unusual nest construction among birds, and within the tanagers it has only been described among species in this clade and two species of Thraupinae (Freeman and Arango, 2010). In our concatenated analyses, the node uniting Coerebinae is strongly supported (Figs. 1 and $2 \mathrm{j} ; 1.0 \mathrm{PP} ; 100 \%$ bootstrap). In addition, this node is supported in genus-level analyses (Barker et al., 2013), strongly supported in our ACO1-I9, cyt $b$, ND2, and RAG1 gene trees, and also supported, although not strongly, by our MB-I2 gene tree (Supplementary Figs. 1-6). Burns et al. (2002) suggested informally using the name Tholospiza (meaning dome finch) for this group, given their dome-shaped nests. Here, we suggest using Coerebinae to designate the subfamily, given that Coerebinae is the oldest family-group name used for any species in this group. Unfortunately, this name has previously been used to describe a clade of nectar-feeding birds that are now known to be paraphyletic (Burns et al., 2003), and the name is still used in some classifications to refer to a monotypic group containing only the Bananaquit (Coereba flaveola). Our subfamily Coerebinae only includes two species (Euneornis campestris and C. flaveola) that were part of this traditional grouping of Neotropical honeycreepers.

Species in Coerebinae show a variety of bill forms, including nectar-feeders (e.g., Coereba, Euneornis), seed-eaters (e.g., Geospiza, Loxigilla, Tiaris), and insect foragers (e.g., Certhidea). Included within this clade are the Darwin's Finches, a classic example of speciation and adaptive radiation (e.g., Grant, 1999; Grant and Grant, 2008; Lack, 1947). Coerebinae also includes nine species endemic to islands in the Caribbean (E. campestris, Loxigilla portoricensis, $L$. violacea, $L$. noctis, L. barbadensis, Melopyrrha nigra, Loxipasser anoxanthus, Tiaris canorus, and Melanospiza richardsoni) and three species in which a large part of the distribution is Caribbean ( $C$. flaveola, T. olivaceus, T. bicolor). The other two non-Darwin's Finch species in Coerebinae ( $T$. fuliginosa and $T$. obscurus) are restricted to South America. The number of Caribbean endemics within Coerebinae is unexpected, given the geographic distribution of other tanagers. Other than the Caribbean members of Coerebinae, only a few other tanager species occur on Caribbean islands and only two other tanagers (Tangara cucullata, Saltator albicollis) are endemic to this area. As was found in previous studies (Burns et al., 2002; Mallarino et al., 2012), our tree indicates that Darwin's finches are embedded within this clade of mostly Caribbean birds, indicating that they are an extension of a radiation that was already occurring among island species in the Caribbean.

Our phylogenies are largely concordant with previously published studies investigating relationships among these species. In 
particular, Mallarino et al. (2012) presented a phylogeny using a similar data set to that presented here. Thus, we do not describe in detail the relationships or taxonomic history of species in this clade. However, our phylogenies (Figs. 1 and $2 \mathrm{j}$ ), as well as those presented in previous studies (Burns et al., 2002; Mallarino et al., 2012; Petren et al., 2005), indicate that several genera are paraphyletic and need taxonomic revision. For example, as in Petren et al. (2005), we found that the two species of Certhidea do not form a monophyletic clade, with $C$. fusca more closely related to the rest of the Darwin's Finches than it is to C. olivacea. However, this relationship was not strongly supported in our data set. If additional data continue to uphold paraphyly, retaining Certhidea for both of these species would be misleading. Thus, a new generic name will be needed for $C$. fusca to reflect accurately the relationship of these species to other birds. For Tiaris and Loxigilla, we found strong support for lack of monophyly. Melopyrrha nigra is embedded within a clade that contains two of the four species of Loxigilla, and this clade has strong support (1.0 PP; 93\% bootstrap). We recommend merging these species into Melopyrrha (type species $=M$. nigra), and retaining Loxigilla (type species $=$ L. noctis) for the other two species of Loxigilla, $L$. noctis and L. barbadensis, which form a strongly supported clade elsewhere in the tree. The five species of Tiaris occur in four different parts of the tree. The type species, T. olivacea, is the sister taxon to all species in Coerebinae except for C. flaveola (1.0 PP; 97\% bootstrap). Because T. olivacea is the type species for the genus, Tiaris can be retained for T. olivacea. Tiaris bicolor is the sister to Melanospiza richardsoni. This relationship has strong support (1.0 PP; 91\% bootstrap); thus, we recommend merging $T$. bicolor into Melanospiza. T. canorus is not closely related to the other species of Tiaris; we recommend using the available name Phonipara Gray 1850 (type $=T$. canorus) for this species. The two remaining species of Tiaris ( $T$. fuliginosa and T. obscurus) are sister species in another part of the tree. We are not aware of an available name for these species; therefore, a new generic name will be required under the classification scheme described above.

For Geospiza and Camarhynchus, we only included cyt $b$ from one individual for each species. Because these species do not sort into monophyletic groups based on their cyt $b$ sequence (Petren et al., 2005; Sato et al., 1999), we caution that the branching order in the trees presented here (Fig. 2j; Supplementary Fig. 1) should not be taken to indicate relationships of those species. Furthermore, Zink (2002) presented an analysis that clouds the species status of forms within Geospiza and within Camarhynchus. Genomic data from multiple individuals of each putative species are likely needed to fully understand evolutionary patterns and species-level relationships within Geospiza and Camarhynchus.

\subsubsection{Tachyphoninae, Bonaparte, 1853; the Ornamented Tanagers}

This clade contains 31 species that are mostly distributed in the Neotropical lowlands (Fig. 3a). The majority of species in this group have long been considered tanagers; however, Volatinia jacarina, Rhodospingus cruentus, and the two species of Coryphospingus have traditionally been considered New World sparrows (Paynter and Storer, 1970). There are 10 genera in this clade, with most species belonging to either Tachyphonus ( 8 species) or Ramphocelus (9 species). Both Tachyphoninae and Ramphocelinae have been used as subfamily names in the past to describe some of the species in this clade. Both of these names were used in the same publication; we act as first revisers in selecting Tachyphoninae Bonaparte, 1853 to take precedence over Ramphocelinae Bonaparte, 1853 as the name of this subfamily. Most species in Tachyphoninae have social ornaments such as crests, enlarged lower mandibles, brightly colored carotenoid patches, white plumage patches, and partially concealed feather patches on the crown. Thus, we suggest a common name of 'ornamented tanagers' for describing this group. The presence of these plumage and bill ornaments and their associated displays (Isler and Isler, 1999; Moynihan, 1962, 1966; Willis, 1985), as well as the marked sexual dichromatism of most species, suggest that sexual selection is strong in this group. Identifying this group as a novel clade should facilitate the study of these features and their associated behaviors.

Tachyphoninae is strongly supported in our concatenated BEAST (0.98 PP) and species tree analyses (Barker et al., 2013). Although Tachyphoninae is monophyletic in our ML tree, our bootstrap analysis recovered this clade in only $54 \%$ of replicates. There are two subclades within Tachyphoninae, which are both strongly supported in Bayesian and ML analyses (Fig. 3a) as well as genus-level analyses (Barker et al., 2013). This basal split involves a small clade (1.0 PP; 73\% bootstrap) containing species in Conothraupis, Volatinia, and Creurgops and a large clade (1.0 PP; $100 \%$ bootstrap) that was termed the 'lowland clade' by Burns and Racicot (2009).

Within the smaller clade, the two species of Creurgops are sister to each other with strong support, as found in previous studies. A close relationship between Creurgops and either Volatinia or Conothraupis has not been previously suggested. However, previous linear classifications (e.g., Hellmayr, 1936; Sclater, 1886; Storer, 1970) have arranged Creurgops near other genera of Tachyphoninae (e.g., Lanio, Tachyphonus, Eucometis, Trichothraupis). The presence of elongated head feathers (i.e., a crest) in Creurgops verticalis also supports the placement of Creurgops within Tachyphoninae. Within Tachyphoninae, 13 of the 31 species have crests. Otherwise, crests are relatively rare in tanagers and are found only in Charitospizinae (Section 4.3.2) and a few species of Thraupinae (Section 4.3.15).

In both ML and Bayesian analyses, Creurgops is sister to a clade containing Conothraupis speculigera and Volatinia jacarina. Bledsoe (1988) was the first to show that $V$. jacarina, the sole member of Volatinia, was a tanager based on DNA hybridization data. Subsequent sequencing studies have confirmed this finding. However, the placement of Volatinia within tanagers has remained unsettled, with the species most often considered closely related to Sporophil$a$ (Clark, 1986; Paynter and Storer, 1970; Sibley and Monroe, 1990) or the Darwin's finches (Steadman, 1982). Previous mtDNA analyses that included some tanagers (Burns et al., 2003; Weir et al., 2009) recovered Volatinia as the sister to Conothraupis speculigera, and we confirm that finding in the present study using more species and genes. The placement of Volatinia within the broader Tachyphoninae agrees with similarities in plumage and overall appearance. Many other species in Tachyphoninae have mostly bluish black plumage like Volatinia, and Volatinia is reminiscent of a small version of Tachyphonus. Volatinia also has white underwing coverts, a character it shares with $C$. speculigera and some species of Tachyphonus.

The phylogenetic position of Conothraupis in relation to the tanagers has been unclear, with most linear classifications including it near the beginning of tanagers alongside genera of uncertain relationships. Storer (1960) remarked on the similarities of Conothraupis with seedeaters, especially Sporophila luctuosa. He considered its relationship to other species undetermined, but most likely close to Schistochlamys and Neothraupis. Conothraupis includes two species, but we were only able to sample $C$. speculigera. In our phylogenies, C. speculigera is clearly part of Tachyphoninae, and this position is also supported by several morphological features. Males have glossy blue-black plumage that is similar to species in Tachyphonus, Ramphocelus, and Volatinia. The female plumage of $C$. speculigera is largely yellow or olive, similar to that of Lanio, Eucometis, and females of some species of Tachyphonus and Ramphocelus. Furthermore, like many other members of Tachyphoninae, adult male $C$. speculigera have elongated head feathers that form a crest. In $C$. speculigera, the crest is rudimentary (Bond, 1951) and when raised by the male during singing bouts 
(Ingels, 2007), an otherwise concealed white patch (Lebbin, 2005, 2006; Witt, 2005) is exposed.

Despite these similarities to Tachyphoninae, the overall plumage color and pattern of $C$. speculigera is very similar to a distantly related species, Sporophila luctuosa (Storer, 1960; Witt, 2005). These similarities include black upperparts, white underparts, and a white wing speculum. Witt (2005) demonstrated that these similarities were likely the result of interspecific visual mimicry between these two species. Our study provides the phylogenetic evidence needed to support this hypothesis; these two species are distantly related to each other in our trees (Fig. 3a and b). Thus, we confirm two cases of interspecific mimicry in tanagers, C. speculigera/S. luctuosa as well as Orchesticus/Philydor rufum in Orchesticinae (Section 4.3.3).

We were unable to sample the other species of Conothraupis, $C$. mesoleuca, previously known from only a single specimen but recently rediscovered (Buzzetti and Carlos, 2005). Based on observations of newly-discovered individuals, Candia-Gallardo et al. (2010) argue that this species might be closely related to the White-naped Seedeater (Dolospingus fringilloides), which belongs to Sporophilinae (Section 4.3.12) in our phylogeny. Similarities between the two species include voice (Ridgely and Tudor, 2009), female plumage, bill color and shape, and morphological measurements (Candia-Gallardo et al., 2010). However, CandiaGallardo et al. (2010) also report that the call notes of C. mesoleuca are similar to those of the sympatric Ramphocelus carbo, which our trees show belongs to Tachyphoninae. Thus, genetic studies are needed to clarify the relationship of $C$. mesoleuca to other tanagers.

The remaining species in Tachyphoninae form a clade of mostly lowland tanagers. The phylogenetic relationships of these species were studied by Burns and Racicot (2009) using ND2 and cyt $b$ sequences. Because Burns and Racicot (2009) discuss the taxonomic history of these species, we do not discuss it here. The additional data included in the current study produced largely congruent results, with most nodes receiving increased posterior probabilities and maximum likelihood support. However, Clade "B" of Burns and Racicot (2009) was not recovered in our study. Instead, some of the species in this clade (Coryphospingus, Rhodospingus, Lanio, and Tachyphonus delatrii) are more closely related to a clade containing T. phoenicius, T. rufus, T. coronatus, and species in Ramphocelus than they are to other members of this group. Thus, the suggestion by Burns and Racicot (2009) to merge these species into a broad genus Lanio is not supported by current results. Burns and Racicot (2009) suggested several alternatives to reconcile the generic taxonomy with the phylogeny. Of those proposed, we recommend retaining Tachyphonus (type species $=$ T. rufus) for the clade containing T. phoenicius, $T$. coronatus, and T. rufus, and using three new generic names, one for $T$. delatrii, one for $T$. surinamus and one for the clade containing for T. cristatus, T. rufiventer, and T. luctuosus. The current usage of the remaining generic names in Tachyphoninae can be retained.

\subsubsection{Sporophilinae Ridgway, 1901; the Seedeaters}

This clade contains 39 species that are currently classified into three genera. The 32 species that we included in this study form a monophyletic group with strong support (Figs. 1 and $3 \mathrm{~b}$; $1.0 \mathrm{PP} ; 100 \%$ bootstrap). Our cyt $b$, ND2, ACO1-I9, and MBI2, RAG1 gene trees also strongly supported the monophyly of this group (Supplementary Figs. 1-6), as do genus-level analyses (Barker et al., 2013). Although this clade has historically been included within the New World sparrows (Paynter and Storer, 1970), here we demonstrate conclusively that it is part of the tanagers. Most of the species within this subfamily are part of Sporophila, whose name reflects their granivorous diet. Six additional species are included in Oryzoborus, and Dolospingus is monotypic. Seedeaters and seed-finches in these genera are small bodied with conical bills and melanin-based plumage, and are widespread from southern Texas to southern Argentina.

Mason and Burns (2013) recently studied phylogenetic relationships within this group. The relationships inferred here are largely congruent with the phylogeny presented by Mason and Burns (2013), which was based solely on ND2 and cyt $b$. Because Mason and Burns (2013) discussed the taxonomic history of this subfamily, we do not repeat that information here. Our data recapitulate the findings of Mason and Burns (2013) that Sporophila is paraphyletic as currently described, with Dolospingus and Oryzoborus embedded within Sporophila. We follow their suggestion in recommending that these three genera be lumped into a monophyletic, broadly-defined Sporophila.

Between the current study and Mason and Burns (2013), no nodes show strongly supported conflict. However, there are some differences in topology and taxonomic sampling. We did not include $S$. bouvreuil, which was included in Mason and Burns (2013). Also, the present study included S. frontalis, which was excluded from Mason and Burns (2013). The inclusion of this species resulted in a topological difference. In our Bayesian trees, we inferred a sister relationship between $S$. frontalis and Dolospingus fringilloides, whereas Mason and Burns (2013) found a strongly supported sister relationship between $D$. fringilloides and a clade containing $S$. luctuosa, S. caerulescens, and S. nigricollis (1.0 PP).

Another difference between the present study and Mason and Burns (2013) is in the placement of Oryzoborus funereus and 0 . angolensis with respect to the other species in Oryzoborus. Mason and Burns (2013) inferred a strongly supported clade containing all six Oryzoborus species. Our cyt $b$ and ND2 gene trees also recovered a strongly supported Oryzoborus (Supplementary Figs. 1 and 2). In contrast, our concatenated analyses (Fig. 3b) rendered Oryzoborus polyphyletic, wherein 0 . crassirostris, 0 . atrirostris, 0 . maximiliani, and $O$. nuttingi form a clade that is distantly related to $O$. funereus and $O$. angolensis. However, none of the nodes separating these two clades were strongly supported. The lack of monophyly in our concatenated analyses appears to be driven by ACO1-I9. We sampled two species of Oryzoborus for this gene, O. angolensis and 0 . crassirostris, and these two species appear in different parts of this gene tree (Supplementary Fig. 3). However, relatively few taxa within this subfamily were sampled for ACO1-I9 or other nuclear genes; thus, sparse taxon sampling could be driving the polyphyletic arrangement. Because these species share morphological as well as mtDNA characters, we consider Oryzoborus to represent a monophyletic lineage unless additional data from other nuclear genes also demonstrate non-monophyly.

\subsubsection{Poospizinae, Wolters, 1980; the Warbler Tanagers}

This large clade contains 44 species currently classified into 12 genera. The node uniting Poospizinae was strongly supported in our concatenated analyses (0.97 PP; $98 \%$ bootstrap; Fig. 4), as well as the generic-level trees of Barker et al. (2013). The cyt $b$ and ND2 gene trees also strongly supported this clade (Supplementary Figs. 1 and 2). About half the species have historically been classified with the tanagers, and the remaining species with New World sparrows, including all members of Poospiza, Compsospiza, Xenospingus, Piezorina, Urothraupis, and Donacospiza. Our analyses show that all of these species are tanagers.

The subfamily is dominated by two relatively large genera (Poospiza and Hemispingus); most other genera are monotypic. Species in Poospiza are commonly referred to as warbling-finches due to their melodious songs. Cypsnagra hirundinacea, some species of Hemispingus, and some species of Thlypopsis are known for their complex vocal displays, often given as duets, trios, or in larger groups (Hilty, 2011). Species in Hemispingus are morphologically and behaviorally similar to, and often confused with, woodwarblers in Basileuterus (Hilty, 2011; Isler and Isler, 1999). 
Although species in Hemispingus vary in bill size, many species of this and other genera in the subfamily (e.g., Thlypopsis, Nephelornis, Urothraupis, Cnemoscopus) have relatively thin, warbler-like bills. Thus, the name 'warbler tanagers' provides a convenient way to refer to this diverse group, considering the warbling songs of some species and the warbler-like morphology of others.

The data sets of the current study and Shultz and Burns (2013) are largely the same; therefore, topological relationship within Poospizinae presented in the current paper are largely concordant with those presented in Shultz and Burns (2013). Support is very similar between the two studies, and no strongly supported nodes conflict. There are only three topological differences between Bayesian trees presented in Shultz and Burns (2013) and those of the current paper. These changes involve the positions of Hemispingus reyi, Poospiza thoracica, and $P$. torquata. Of these topological changes, only the change in position of $P$. torquata results in a significant change in support values. In the analyses presented here, the position of $P$. torquata results in an increase in support from 0.49 to $0.95 \mathrm{PP}$ for the clade containing P. torquata, H. trifasciatus, $P$. melanoleuca, and $P$. cinerea.

Shultz and Burns (2013) discussed the taxonomic history of species in this subfamily; thus, that information is not repeated here. However, we note that our topology will require extensive generic revision within the group. In particular, the large genera Hemispingus and Poospiza are polyphyletic with respect to each other; therefore, for the classification to be consistent with strongly-supported monophyletic lineages, several new genera will need to be named. In other cases, previously used generic names can be resurrected to accommodate our topology. For example, Pseudospingus Berlepsch and Stolzmann, 1896 (type $=H$. xanthophthalmus) is available for the clade containing Hemispingus verticalis and $H$. xanthophthalmus. Similarly, Sphenops Sclater, 1862 (type $=H$. frontalis) is available for $H$. frontalis and $H$. melanotis, Orospingus Riley, 1922 (type $=H$. goeringi) is available for $H$. goeringi and H. rufosuperciliaris, and Poospizopisis Berlepsch, 1893 (type $=$ Poospiza caesar) is available for $P$. hypochondria and $P$. caesar. Microspingus Taczanowski, 1874 (type species = Hemispingus trifasciatus) is available for the large clade containing $H$. trifasciatus, Poospiza cabanisi, P. erythrophrys, P. alticola, P. torquata, P. melanoleuca, and $P$. cinerea. We were unable to include a sample of $P$. lateralis. $P$. cabanisi was recently split from $P$. lateralis (Assis et al., 2007); thus, the two species are likely related, and we would place both in Microspingus. We recommend using the name Thlypopsis Cabanis, 1851 for the clade containing all species of Thlypopsis, Pyrrhocoma ruficeps, and $H$. superciliaris (all three genera date to 1851). Under the above described classification scenario, Poospiza would be retained for the clade containing $P$. boliviana, $P$. ornata, and $P$. whitii/nigrorufa, and Compsospiza would be retained for C. baeri and C. garleppi. In addition, the monotypic Piezorina, Xenospingus, Cnemoscopus, Donacospiza, Cypsnagra, Urothraupis, and Nephelornis would be retained; however, Hemispingus would no longer be used. If all of the above taxonomic recommendations are followed, new genera will be needed for $P$. hispaniolensis, for $P$. rubecula, for the clade containing $H$. reyi, $H$. atropileus, $H$. calophrys, and $H$. parodii, and for $P$. thoracica. An alternative classification scheme could involve lumping species into larger genera; however, to be consistent with strongly-supported nodes, these genera would be morphologically diverse.

\subsubsection{Diglossinae, Sclater, 1875 , the Highland Tanagers}

With 64 species and 14 genera, this clade is one of the largest and most morphologically diverse tanager subfamilies. Diglossinae, as defined in this paper, has not previously been recognized as a clade because no prior study has comprehensively sampled the tanagers. However, some earlier studies that sampled fewer species (Barker et al., 2013; Burns et al., 2002, 2003; Campagna et al., 2011; Yuri and Mindell, 2002) have recovered aspects of this clade. Here, we found strong support in our concatenated phylogenies (1.0 PP; 100\% bootstrap; Figs. 1 and 5) for a clade containing Conirostrum, Oreomanes, Sicalis, Catamenia, Diglossa, Idiopsar, Xenodacnis, Haplospiza, Acanthidops, Nesospiza, Rowettia, Melanodera, one of the two species of Diuca, and eight of the 11 species of Phrygilus. A few of these species were historically considered tanagers (species in Oreomanes and Diglossa); however, most were traditionally considered members of Parulidae (Conirostrum) or Emberizidae (the remaining genera) (Fig. 5; Lowery and Monroe, 1968; Paynter and Storer, 1970). Even among the tanagers, the bill morphologies and feeding behaviors of species in Diglossinae are particularly diverse. There are nectar feeders (Diglossa), finchbilled seed-eaters (e.g., Nesospiza, Sicalis, Catamenia, Haplospiza), a bark gleaner (Oreomanes), arthropod feeders (Conirostrum), a bamboo specialist (Acanthidops), an aphid and nectar feeder (Xenodacnis), and a boulder field specialist (Idiopsar). Although some species occur only in the lowlands, over $80 \%$ have their center of abundance at $900 \mathrm{~m}$ or higher, and over $75 \%$ occur above $2500 \mathrm{~m}$ (Parker et al., 1996). Thus, we recommend the name "highland tanagers" to describe this group.

The genus Phrygilus is one of the most polyphyletic in our tanager phylogeny, with members appearing in four distinct clades, three of which are in Diglossinae. Previous workers (e.g., Fjeldså, 1992; Lowe, 1923) have appreciated the heterogeneous nature of Phrygilus, and Campagna et al. (2011) were the first to demonstrate polyphyly across the group using molecular characters. In general, our results are concordant with those of Campagna et al. (2011), who sampled fewer species of tanagers, but found the same four distinct Phrygilus clades. These four clades have also been identified on the basis of plumage differences (Ridgely and Tudor, 1989) and agree to some extent with skeletal differences (Webster and Webster, 1999). For convenience, we refer to these groups as the "gray sierra-finches" (P. unicolor, P. plebejus), the "yellow-billed sierra-finches" (P. alaudinus, P. fruticeti, P. carbonarius), the "hooded sierra finches" ( $P$. atriceps, $P$. gayi, $P$. punensis, $P$. patagonicus), and the "gray and white sierra-finches" ( $P$. dorsalis, $P$. erythronotus). The yellow-billed Sierra finches were discussed earlier (Porphyrospizinae, Section 4.3.6). The other three sierra-finch clades belong within Diglossinae, but are not closely related to each other within Diglossinae. In the following paragraphs, we discuss relationships of these three clades in the context of their nearest relatives. Overall, our results with regard to species-level relationships of Phrygilus are consistent with those of Campagna et al. (2011), to the extent that the two studies sampled taxa in common. However, our more complete sampling does allow us to identify with confidence the closest relatives to each of these clades.

Within Diglossinae, the clade containing the conebills Conirostrum and Oreomanes is sister to all remaining species (1.0 PP; $100 \%$ bootstrap). Conirostrum itself has been classified in a variety of different groups including warblers (e.g., Howard and Moore, 1991; Lowery and Monroe, 1968), tanagers (e.g., American Ornithologists' Union, 1998; Sibley and Monroe, 1990), and honeycreepers (e.g., Hellmayr, 1935). The warbler-like bills of all Conirostrum and nectar-feeding habits of some species (Hilty, 2011; Vogt, 2006) have contributed to this taxonomic confusion. However, all modern molecular studies have placed Conirostrum within the tanagers (e.g., Barker et al., 2013; Burns et al., 2003; Campagna et al., 2011; Lovette and Bermingham, 2002; Yuri and Mindell, 2002). The Giant Conebill (Oreomanes fraseri) has had a similar taxonomic history, and has been shuffled among warblers, tanagers, and honeycreepers by various taxonomists. Oreomanes fraseri is a Polylepis specialist that has a larger, more rounded body shape than conebills in the genus Conirostrum, and closely resembles nuthatches in its morphology, tree-climbing, and bark-probing behaviors (Mason and Burns, 2010; Vuilleumier, 1984). Oreomanes was often placed 
adjacent to Conirostrum in early linear arrangements (Hellmayr, 1935; Sclater, 1886), implying a close relationship between these two genera, and Sclater (1886) and Ridgway (1902) specifically noted the similarity between them. In particular, Sclater (1886) called Oreomanes "an exaggerated form of Conirostrum"; however, other later arrangements (Howard and Moore, 1991; Lowery and Monroe, 1968; Storer, 1970) did not consider these taxa to be closely related. This discrepancy was likely due to a study of Coerebidae by Beecher (1951) that placed Conirostrum with the warblers and Oreomanes with the tanagers. Similarities between Oreomanes and Diglossa, another member of our Diglossinae, have also been recognized (George, 1964; Sclater, 1860; Storer, 1970). Schulenberg (1985) described a hybrid between Oreomanes and C. ferrugineiventre. Based on this hybrid and similarities in plumage color, plumage pattern, and body shape, Schulenberg (1985) provided the most detailed argument for a close relationship between Oreomanes and Conirostrum. He proposed two alternative possibilities: either Oreomanes and Conirostrum are sister taxa, or Conirostrum is paraphyletic with Oreomanes being more closely related to some Andean species of Conirostrum. Fjeldså (1992) suggested a sister relationship between Oreomanes and C. ferrugineiventre based on similar coloration and shared behavior of feeding on Polylepis trunks. Molecular phylogenetic analyses have confirmed that Oreomanes is a tanager (Burns, 1997; Burns et al., 2003), and confirmed a close relationship between Oreomanes and Conirostrum (Barker et al., 2013; Burns et al., 2003; Campagna et al., 2011; Lovette and Bermingham, 2002; Yuri and Mindell, 2002). Burns et al. (2003) sequenced cyt $b$ from a broad sampling of tanagers, Neotropical finches, and species previously considered part of Coerebidae, and they found that Oreomanes was most closely related to the two species of Conirostrum (C. bicolor and C. speciosum) included in their study. Similarly, Campagna et al. (2011) sampled cyt $b$ from five species of Conirostrum and also found that Conirostrum was paraphyletic with respect to Oreomanes. In the present study, we include all species of Conirostrum and confirm both the monophyly of Conirostrum + Oreomanes (1.0 PP; $100 \%$ bootstrap) and the paraphyly of Conirostrum with respect to Oreomanes. These results are supported by separate gene analyses (Supplementary Figs. 1-6). Our findings agree with the prediction of Schulenberg (1985) that Oreomanes is closely related to a clade of mostly Andean Conirostrum. Although $O$. fraseri and $C$. ferrugineiventre have hybridized, we did not find that these two species were sister taxa. Instead, 0 . fraseri is sister to a clade of five species of Conirostrum (referred to as Group A in Ridgely and Tudor (1989)), one of which is C. ferrugineiventre. Thus, Oreomanes is a specialized Conirostrum, and the morphological and behavioral differences of Oreomanes are best interpreted as recently evolved adaptations. Given that the position of Oreomanes renders Conirostrum paraphyletic, we recommend merging Oreomanes with Conirostrum, which has taxonomic priority.

Within Conirostrum, species are generally divided into two groups based on differences in habitat preference (e.g., Ridgely and Tudor, 2009). The four species of lowland conebills (C. bicolor, C. margaritae, C. leucogenys, $C$. speciosum) have historically been placed in Ateleodacnis (e.g., Hellmayr, 1935) and the six species of highland conebills ( $C$. sitticolor, C. cinereum, C. tamarugense, C. ferrugineiventre, $C$. rufum, and C. albifrons) are considered to be members of true Conirostrum. However, Zimmer (1942) was unable to find morphological characters to justify this separation. Our phylogenies also do not support a monophyletic lowland clade. Instead, two of the lowland species (C. leucogenys and $C$. speciosum) are more closely related to highland species than to the other lowland species (C. margaritae and C. bicolor). Support for this relationship is high (1.0 PP; 93\% bootstrap). Thus, the use of Ateleodacnis to recognize a clade of lowland conebills is not warranted. However, the highland species, together with Oreomanes, do form a clade with respect to the lowland species. This topology suggests that the highland distribution of these conebills was derived from a lowland ancestor.

In our phylogenies, the earliest split within Conirostrum is between a clade containing $C$. margaritae and $C$. bicolor and a clade containing the remaining species. These two clades are well differentiated genetically from each other and each is strongly supported. This early division within Conirostrum was also recovered in the phylogenies of Campagna et al. (2011), who sampled fewer species than in our study. This division corresponds with habitat differences; C. bicolor and C. margaritae inhabit riverine forests, specializing on river islands.

The Tamarugo Conebill (C. tamarugense) is a relatively recently described species (Johnson and Millie, 1972), classified as vulnerable by the IUCN (BirdLife International, 2013b). The species has a very limited distribution in southwestern Peru and northern Chile. Little has been written on its potential relationships, but Mayr and Vuilleumier (1983) considered this species closely related to C. rufum and C. ferrugineiventre, and Fjeldså (1992) considered it to be sister to $C$. cinereum. We report the first genetic data for this species and show that it is well differentiated from the other species of Conirostrum and its sister taxon is a clade containing C. rufum and $C$. cinereum.

Conirostrum is sister to a large clade composed of the remaining Diglossinae. This clade can be further divided into two strongly supported main clades. One of these clades contains the yellow finches (Sicalis), the two species of bridled finches (Melanodera), three species of tanagers that occur on islands in the South Atlantic (Rowettia, Nesospiza), and the hooded Sierra-finches (Phrygilus). All species in this clade have yellow in their plumage, thus we refer to them as the "yellow clade" below. The other clade contains the flowerpiercers (Diglossa), four species of sierra-finches (Phrygilus), the Slaty and Uniform Finches (Haplospiza), the Peg-billed Finch (Acanthidops), the White-winged Diuca Finch (Diuca speculifera), the Short-tailed Finch (Idiopsar) and the Tit-like Dacnis (Xenodacnis). All of these species have gray and/or blue in their plumage, thus we refer to them as the "gray/blue clade" below. Both are strongly supported in our analyses (1.0 PP for both clades; $100 \%$ bootstrap for yellow clade, $99 \%$ for gray/blue clade).

Within the "yellow clade", the largest genus is Sicalis with 12 species. Commonly known as yellow finches, these ground-feeding birds are found mostly in open habitats at both high and low elevations. Like other finch-billed tanagers, species in Sicalis were not considered tanagers until relatively recently. Sharpe (1888) divided species in this genus into two groups, with some placed in Pseudochloris near other emberizids and other species in Sycalis (i.e., Sicalis) near old world cardueline finches such as Serinus (currently considered Fringillidae). However, Ridgway (1901a) correctly felt that the species classified as Sicalis by Sharpe (1888) were closely related to those in Pseudochloris. Ridgway (1901a) was also correct in placing Sicalis with other Neotropical emberizines (such as Acanthidops and Haplospiza). The position of Sicalis in Hellmayr's (1938) classification reverts somewhat to that of Sharpe's (1888) in that he placed the genus near species now considered Fringillidae. Meyer de Schauensee (1966), following Tordoff (1954), considered Sicalis to belong with emberizine finches, and subsequent classifications (e.g., Paynter and Storer, 1970) followed this arrangement. DNA analyses, however, have shown that finches in Sicalis, like many other Neotropical Emberizidae, are tanagers (Barker et al., 2013; Bledsoe, 1988; Burns et al., 2002, 2003; Campagna et al., 2011; Klicka et al., 2007).

In our concatenated ML and BEAST analyses, Sicalis is not monophyletic, with $S$. citrina more closely related to species in the "yellow clade" than to other species in Sicalis. However, support for this relationship is not strong ( $0.84 \mathrm{PP}$; $62 \%$ bootstrap). In addition, none of the individual gene phylogenies provides strong 
support either for or against a monophyletic Sicalis (Supplementary Figs. 1-6). Given the lack of strong support for the position of $S$. citrina, additional data are needed to clarify the monophyly of Sicalis. Until then, despite the position of S. citrina in our concatenated phylogeny, we recommend that Sicalis be maintained for all species in the genus, given their morphological and behavioral similarities.

Our data can be used to address several preexisting hypotheses about both relationships within Sicalis and relative distinctiveness of particular taxa. Our phylogenies agree in topology with respect to the four species of Sicalis (S. flaveola, S. luteola, S. luteocephala, S. olivascens) also sampled in the molecular phylogenetic analyses of Campagna et al. (2011). S. taczanowskii has a much thicker bill than other species of Sicalis and has therefore sometimes been classified in its own genus, Gnathospiza (e.g., Hellmayr, 1938; Meyer de Schauensee, 1966). In our trees, S. taczanowskii is embedded within the phylogeny of Sicalis with strong support; therefore, there is no justification for the use of Gnathospiza, and the large bill of this species is best interpreted as yet another case within tanagers of rapid bill evolution. Ridgely and Tudor (1989) divided Sicalis into two groups ("Group A" and "Group B") that mostly corresponded to Sharpe's Sicalis and Pseudochloris. Group A species occur in the arid Pacific slope or are widespread in the lowlands; species in this group include S. taczanowskii, S. flaveola, S. columbiana, S. citrina, S. luteola, and S. raimondii. Group B species are found in the Andes and/or Patagonia and include S. uropygialis, S. luteocephala, S. lutea, S. olivascens, $S$. auriventris, and S. lebruni. With the exception of $S$. citrina, Sharpe's (1888) Pseudochloris correspond to Ridgely and Tudor's (1989) Group B. None of these proposed groupings are monophyletic in our phylogenies. However, Ridgely and Tudor's Group B is monophyletic if $S$. raimondii is included within Group B rather than Group A. Similarly, with the exception of S. citrina and S. raimondii, Pseudochloris would be monophyletic. Sicalis columbiana and S. flaveola have red facial feathers but are not each other's closest relatives, with $S$. columbiana more closely related to the clade containing S. luteola and S. luteiventris. This topological position suggests either red plumage has evolved twice within Sicalis or that red plumage was lost in the lineage leading to S. luteola/S. luteiventris.

Species limits within Sicalis have shifted throughout the classification history of the group, with many forms considered species or subspecies by different taxonomists. For example, Sibley and Monroe (1990) consider S. luteiventris a valid species; however, Clements et al. (2013) consider it a subspecies of S. luteola. Because we had genetic material of $S$. luteiventris, we included it in our analyses. Levels of uncorrected mtDNA sequence divergence between these two taxa average $0.61 \%$, similar to values seen within other species of tanagers. Our phylogeny shows little agreement with regards to previous ideas about superspecies, further emphasizing the prior lack of understanding of species limits and specieslevel relationships in this group. For example, S. olivascens and S. lebruni are considered a superspecies by Sibley and Monroe (1990) and subspecies of the same species by Paynter and Storer (1970); however, these species are only distantly related to each other in our phylogeny. Likewise, S. luteola/S. luteiventris and $S$. raimondii form another superspecies (Sibley and Monroe, 1990), and S. raimondii was long considered a subspecies of S. luteola (Ridgely and Tudor, 1989). However, S. raimondii is more closely related to two species found allopatrically further south in the Andes, S. lutea and S. auriventris. We were unable to include samples of $S$. mendozae, a species recently split from $S$. olivascens (Areta et al., 2012). Based on plumage, structural features, and voice, Areta et al. (2012) consider this species most closely related to S. lebruni.

The yellow clade also includes the four species of sierra-finches that make of up the hooded sierra-finch clade (Phrygilus gayi,
P. atriceps, $P$. punensis, and $P$. patagonicus). These species share similar plumage patterns and colors (Ridgely and Tudor, 1989) and have been considered a superspecies by some authorities (Sibley and Monroe, 1990). In agreement with Campagna et al. (2011), we found strong support for a clade containing these species in our concatenated BEAST and ML analyses (1.0 PP; 100\% bootstrap).

Species limits in the hooded sierra-finch group have fluctuated throughout their taxonomic history, with successive authorities variously classifying the different forms as subspecies or species. Sharpe (1888) considered P. patagonicus synonymous with P. gayi, but considered $P$. atriceps, $P$. gayi, and $P$. punensis as specifically distinct. Hellmayr $(1932,1938)$ argued that $P$. patagonicus was a valid species, but considered the other three hooded sierra-finches part of P. gayi. Both Meyer de Schauensee (1966) and Paynter and Storer (1970) split $P$. atriceps from $P$. gayi because the two species co-occur in Chile without interbreeding (Philippi, 1942), but continued to keep $P$. punensis as a subspecies of $P$. gayi. Based on Vuilleumier (1967), Ridgely and Tudor (1989) split P. punensis from P. atriceps. Subsequent taxonomic treatments (e.g., Sibley and Monroe, 1990; Clements et al., 2013) followed Ridgely and Tudor's (1989) treatment and recognized four valid species of hooded sierra-finches. Hybridization among taxa has contributed to some of this taxonomic confusion; P. gayi is known to hybridize with both P. atriceps (Marín et al., 1989) and P. patagonicus (Vuilleumier, 1991). Vuilleumier (1991) hypothesized a sister taxon relationship between $P$. patagonicus and $P$. gayi, with their speciation associated with a habitat shift. Molecular phylogenetic data support the current taxonomy of the four species. In our study, we found these taxa to be genetically distinct from each other. Furthermore, Campagna et al. (2011) sampled multiple individuals of each species and found each to form a well differentiated clade whose monophyly was strongly supported. Nevertheless, species limits within the group are complex, including at least one population of $P$. gayi that perhaps belongs in P. patagonicus (Jaramillo, 2011d); thus, further intraspecific sampling within this group would help clarify species limits. Although current species limits agree with molecular phylogenetic data, relationships of the four species to each other do not necessarily agree with hybridization patterns or the previous taxonomies described above. Our concatenated tree agrees in topology with the concatenated tree of Campagna et al. (2011), with P. atriceps and P. punensis as sister taxa. Campagna et al. (2011) also found that $P$. gayi is more closely related to the $P$. atriceps/P. punensis clade than to $P$. patagonicus. However, we were not able to recover strong support for placement of $P$. gayi and $P$. patagonicus with respect to the $P$. atriceps/P. punensis clade. Our ML analyses recovered the same topology as Campagna et al. (2011), but without strong support (56\% bootstrap). In contrast, our BEAST analyses show $P$. patagonicus as sister to the $P$. atriceps/P. punensis clade, albeit with weak support (0.48 PP). Phrygilus is polyphyletic, and the type species ( $P$. gayi) is a member of the hooded sierra-finch clade. Thus, we recommend retaining the genus name Phrygilus for these four species.

The hooded sierra-finch clade is sister to a clade containing the two bridled finches (Melanodera) of southern South America and the only tanagers found outside Mexico, Central, or South America (Nesospiza, Rowettia). The two species of Nesospiza and the one species of Rowettia are found on islands in the South Atlantic Ocean. The Nightingale Finch ( $N$. acunhae) and Wilkin's Finch (N. wilkinsi) occur in the Tristan da Cunha archipelago and the Gough Island Finch ( $R$. goughensis) occurs on Gough Island. Tristan da Cunha is about halfway between the southern tips of South America and Africa, with Gough Island $350 \mathrm{~km}$ southeast of the Tristan group (Ryan, 2007). Relationships among these species were reported in Ryan et al. (2013) using some of the same data as reported in the current study, and the results of these two studies are concordant. The topologies of both studies imply two 
colonization events by tanagers of these South Atlantic islands. Ryan et al. (2013) discussed the taxonomic implications of phylogenetic relationships in this part of the tree.

Within the gray/blue clade, the largest genus is Diglossa. The 18 species in Diglossa are known as flowerpiercers, and they have adaptations that allow them to obtain floral nectar without pollination. These adaptations include a specialized hook at the tip of the maxilla used to hold the corolla of a tubular flower while the sharp lower mandible is used to pierce the flower base (Skutch, 1954). Mauck and Burns (2009) studied relationships among the flowerpiercers using cyt $b$ and ND2. The current study used these data as well as additional nuclear sequences. The taxonomic history of the group was described in Mauck and Burns (2009) and is not repeated here. The topological relationships found in the current study are largely congruent with those of Mauck and Burns (2009); therefore, relationships among these taxa are also not described in detail here. As in Mauck and Burns (2009), we found a rapid radiation of the four species in the carbonaria superspecies. Our concatenated BEAST tree differs in one place from the trees of Mauck and Burns (2009). The Tepui species, D. major and D. duidae, were weakly supported as monophyletic in Mauck and Burns (2009); in the current study, we do not recover an exclusive clade containing $D$. duidae and $D$. major. Instead, weak support is found for a clade containing D. major and six other species of Diglossa. Our results agree with those of Mauck and Burns (2009) in that recognition of Diglossopis is not warranted, given the position of D. indigotica. Mauck and Burns (2009) were unable to identify the sister group to Diglossa, although they did identify a large clade containing Diglossa as well as Acanthidops, Catamenia, Conirostrum, Haplospiza, Idiopsar, Melanodera, Oreomanes, Phrygilus, Sicalis, and Xenodacnis. This clade is consistent with our Diglossinae and aspects of this clade were also recovered in Burns et al. (2003). With the more extensive sampling of the current study, we were able to identify Catamenia as the sister to Diglossa, with strong support (1.0 PP; $98 \%$ bootstrap).

Catamenia consists of three species of Andean birds commonly known as seedeaters: the Paramo Seedeater ( $C$. homochroa), the Plain-colored Seedeater ( $C$. inornata), and the Band-tailed Seedeater ( $C$. analis). The sister relationship between Catamenia and Diglossa is notable for a couple of reasons. The bill shapes of Diglossa and Catamenia are very different; the three species of Catamenia have conical, seed-eating bills whereas Diglossa flowerpiercers have longer, thinner bills specialized for nectar feeding. Although the bill shapes are different, at least one species of Catamenia ( $C$. analis) has been observed stealing nectar from flowers, similar to Diglossa (Wester and Claßen-Bockhoff, 2006). This observation suggests a deeper evolutionary origin for this behavior than previously recognized.

Like other seed-eating Neotropical tanagers, Catamenia was typically classified in families of other seed-eating birds (e.g., Sharpe, 1888; Hellmayr, 1938; Paynter and Storer, 1970). However, Sibley and Monroe (1990) classified it with tanagers, and subsequent DNA phylogenies have confirmed this (e.g., Barker et al., 2013; Burns et al., 2002, 2003; Campagna et al., 2011; Klicka et al., 2007). Most pre-molecular classifications have considered Catamenia to be related to Sporophila, either placing species of Catamenia within Sporophila (e.g., Sharpe, 1888) or adjacent to Sporophila (e.g., Hellmayr, 1938; Paynter and Storer, 1970). Our phylogenies show that despite their shared seed-eating habits, Catamenia and Sporophila are not closely related. In contrast to most classifications, Ridgely and Tudor (1989) considered Catamenia near Idiopsar, Phrygilus, Diuca, and Melanodera. This arrangement more closely matches our phylogenies. Some of the analyses of Burns et al. (2003) placed Catamenia and Diglossa as sister taxa, although support for this relationship was not strong $(<60 \%)$. The increased data and character sampling of the current study increased support for this relationship and confirms that these two genera are each other's closest relatives. Within Catamenia, we found that $C$. inornata and $C$. homochroa are more closely related to each other than they are to $C$. analis. This agrees with shared plumage coloration and plumage molt sequences of these two species (Dickerman, 1986; Fjeldså, 1992).

The Catamenia/Diglossa clade is most closely related to a clade of 10 species that includes Xenodacnis parina, the gray and white sierra-finches (Phrygilus erythronotus and P. dorsalis), Idiopsar brachyurus, Diuca speculifera, the gray sierra-finches ( $P$. plebejus and $P$. unicolor), the two species of Haplospiza, and Acanthidops bairdi. Monophyly of this clade was strongly supported (1.0 PP; $96 \%$ bootstrap). The Tit-like Dacnis, $X$. parina, has many unusual features; therefore, it has been classified in its own genus with an uncertain taxonomic position (Fjeldså, 1992; George, 1964; Zimmer, 1942). Some of the unique features of this high elevation species include bright blue plumage of males, marked sexual dichromatism, and a relatively small bill given overall body size. Earlier taxonomies (e.g., Hellmayr, 1936; Sclater, 1886) considered it part of the Neotropical honeycreeper family Coerebidae. This family has subsequently been dissolved (Burns et al., 2003), and DNA phylogenies indicate that this species belongs with the tanagers (Barker et al., 2013; Burns et al., 2003). Burns et al. (2003) had less complete taxon sampling than the present study and showed strong support that Xenodacnis was closely related to species in Catamenia, Diglossa, Haplospiza, and Acanthidops, with some evidence for Sicalis, Oreomanes, and Conirostrum being included in this clade as well. Results of the current study are consistent with these findings with respect to species sampled in common between the two studies. Although the bright blue coloration of males is similar to some species of Diglossa (Fjeldså, 1992), our trees show that Xenodacnis is embedded within a clade of mostly gray-plumaged birds (some Phrygilus, Idiopsar, D. speculifera, Haplospiza, and Acanthidops).

Among these species, Xenodacnis is most closely related to a clade containing $I$. brachyurus, D. speculifera, and the two species of gray and white sierra-finches (P. erythronotus and $P$. dorsalis). Campagna et al. (2011) did not sample D. speculifera, but otherwise also identified a strongly supported clade containing I. brachyurus, $P$. erythronotus, and $P$. dorsalis. Our analyses are the first to identify an exclusive clade containing these four species; however, Fjeldså (1992) noted plumage and distribution similarities that these species share. All species inhabit high arid puna or very high altitudes in the puna and they share the plumage synapomorphies of white throat and white mottling below the eye (Fjeldså, 1992). In contrast, the other species of Diuca, D. diuca, is found at lower elevations in semi-open habitat. Voice differences and locomotion differences also suggest that the two Diuca species are not closely related (Jaramillo, 2011e). Our phylogenies are congruent with these differences and show the two Diuca species are distantly related, with $D$. speculifera belonging to Diglossinae and $D$. diuca belonging to Thraupinae (Section 4.3.15).

In our phylogenies, the closest relative to $D$. speculifera is the Short-tailed Finch (I. brachyurus), also known as the Andean Boulder-finch (Lloyd et al., 2005), a relatively large bodied finch with a sharply-pointed bill that occurs in the high elevation Andean grasslands. The bill shape of Idiopsar is quite different from that D. speculifera; however, the species occur in the same habitat, have similar call notes (Fjeldså and Krabbe, 1990; Ridgely and Tudor, 2009), share similar posture, and have similar foraging behaviors (Lloyd, 2009). Levels of pairwise sequence divergence between these two species are remarkably low. We found no differences in the nuclear genes sequenced and levels of uncorrected mtDNA sequence divergence $(0.42 \%)$ are similar to what is seen within other species of tanagers. Given this low level of sequence variation, we sequenced multiple individuals of each species (data not shown) and confirmed this result. These data suggest 
either recent hybridization between the two species, or rapid evolution of morphological characters. More research is needed to distinguish between these scenarios.

The $D$. speculifera/Idiopsar clade is most closely related to the two gray and white sierra-finches, $P$. erythronotus and $P$. dorsalis. These two species are both high altitude specialists with nearly allopatric distributions (Ridgely and Tudor, 1989). They were considered a superspecies by Sibley and Monroe (1990) and are known to interbreed (Fjeldså and Krabbe, 1990), suggesting only one species is involved. Our genetic results confirm the close relationship of these two species, and Campagna et al. (2011) sampled multiple individuals of each and recovered reciprocal monophyly, as expected for taxa representing welldifferentiated species.

Because Phrygilus and Diuca are polyphyletic, a new generic taxonomy is needed for the four tanagers in this part of our phylogeny. The type species of Phrygilus, P. gayi, belongs to the hooded sierra-finch clade described above. Thus, we recommend merging these four species into Diuca Reichenbach 1850 (type $=D$. speculifera), which has taxonomic priority over Idiopsar Cassin 1866 (type $=I$. brachyurus). Merging these species has some precedent in the literature. Paynter and Storer (1970) noted that Diuca was possibly congeneric with Idiopsar, and Wolters (1975-1980) included all four in Diuca. Alternatively, both Diuca and Idiopsar could be retained as monotypic genera, but a new genus name would be needed for $P$. erythronotus and $P$. dorsalis.

The Xenodacnis - Idiopsar clade is most closely related to a clade containing Acanthidops bairdi, the two species of Haplospiza, and the two species of gray sierra-finches (Phrygilus plebejus and P. unicolor). The clade uniting these species has strong support in our molecular phylogenies (1.0 PP; $98 \%$ bootstrap) and earlier workers suspected a close relationship among these species based on other characters. For example, the two Phrygilus species share streaked female plumage with one other (Fjeldså, 1992), as well as plumage and structural similarities with Haplospiza (Jaramillo, 2011f, 2011g). In addition, a close relationship between Acanthidops and Haplospiza has long been suspected (e.g., Ridgway, 1901a) based on similarities in plumage and other characters. Paynter and Storer (1970) suggested that Acanthidops and Haplospiza were "offshoots" of Phrygilus. Although there are morphological similarities among these five species, Acanthidops and the two Haplospiza species are found in association with bamboo, while the Phrygilus species in this clade are found in high-elevation, open habitats.

Haplospiza and Acanthidops have been classified in alternative ways throughout their taxonomic history. Sibley and Monroe (1990) treated the two species of Haplospiza as a superspecies, and Paynter and Storer (1970) suggested that these two as well as Acanthidops might be conspecific. In contrast, the two Haplospiza species have been considered members of separate genera (e.g., Hellmayr, 1938; Sharpe, 1888), with H. rustica placed in Spodiornis. We sampled both species of Haplospiza for FGB-I5, cyt $b$, and ND2 (Supplementary Figs. 1-6); Haplospiza did not form an exclusive monophyletic group in any of these gene trees. Our BEAST tree using the concatenated data shows that $H$. rustica was more closely related to Acanthidops than to $H$. unicolor, with strong support (0.99 PP; 93\% bootstrap). Thus, we do not recommend retaining Haplospiza as currently used. Instead, Spodiornis Sclater 1866 (type $=H$. rustica) is an available name that can be used for $H$. rustica. Haplospiza can be retained for $H$. unicolor, and Acanthidops can be retained for $A$. bairdi. This approach emphasizes the distinctive upturned and pointed bill of Acanthidops. Alternatively, all three species could be merged into Haplospiza, which has priority over Acanthidops. However, there is not strong support for such a clade and we recommend that each species has its own genus. Because of Phrygilus polyphyly, a name other than Phrygilus is needed for the two gray sierra finches (P. plebejus and $P$. unicolor). We suggest using the available generic name Geospizopsis Bonaparte 1856, which has unicolor as the designated type species.

\subsubsection{Thraupinae, Cabanis, 1847; the Core Tanagers}

With 102 species and 22 genera, Thraupinae is the largest tanager subfamily. It contains many of the species typically associated with the tanagers; therefore, we follow the recommendation of Burns and Naoki (2004) and use the name 'core tanagers' to describe this group. However, there are also nine species in this clade traditionally associated with the New World sparrows, and one is associated with the cardinal-grosbeaks (Fig. 6). We found strong support for monophyly of Thraupinae in our concatenated analyses ( $0.99 \mathrm{PP} ; 86 \%$ bootstrap); monophyly was also supported in the genus-level analyses of Barker et al. (2013). Many core tanager species were included in two prior phylogenetic studies. Burns and Naoki (2004) studied relationships among species in Tangara using cyt $b$ and partial ND2 sequences. Sedano and Burns (2010) expanded this study to include 34 additional species in the core tanager clade and complete ND2 sequences. In the present study, we included five additional species and four additional genetic markers. In general, our results are similar to those reported by Sedano and Burns (2010). None of the nodes that were strongly supported in Sedano and Burns (2010) conflict with those reported in our BEAST and ML trees (Fig. 6). However, support for many nodes increased in our more complete data set. Because the previous studies (Burns and Naoki, 2004; Sedano and Burns, 2010) discussed relationships among these taxa, we do not discuss them in detail here. However, we report the first genetic data for several additional species and comment on their relationships below.

Within Tangara, two additional species were included in the present study, T. rufigenis, a rare species endemic to Venezuela, and $T$. phillipsi, a relatively recently described species (Graves and Weske, 1987). Prior to Burns and Naoki (2004) and Sedano and Burns (2010), Isler and Isler (1999) provided the most comprehensive, recent treatment of Tangara. Isler and Isler (1999) classified species in the genus into 13 species groups based on range, physical appearance, behaviors, and ecology. Isler and Isler (1999) placed T. rufigenis into species group 7 along with T. lavinia and T. gyrola. However, they considered this placement tentative, and suggested that an alternative placement in group 9 (T. ruficervix, $T$. labradorides, and $T$. cyanotis) might be more accurate. Our results indicate that $T$. rufigenis belongs in a clade with $T$. labradorides and $T$. cyanotis; thus, $T$. rufigenis is more closely related to some of the species in Isler and Isler's group 9 than to any other Tangara. T. ruficervix, the other member of Isler and Isler's species group 9, is only distantly related to these species. Sedano and Burns (2010) also identified a close relationship between $T$. labradorides and $T$. cyanotis, but did not sample T. rufigenis. Here, we show that T. rufigenis belongs in this clade as well and is the closest living relative to T. labradorides (1.0 PP; $100 \%$ bootstrap). The close relationship of $T$. labradorides, T. cyanotis, and T. rufigenis was anticipated by some workers before Isler and Isler (1999). When T. rufigenis was originally described, Sclater (1856) indicated it was most similar to T. labradorides. Although Sclater's later classification (Sclater, 1886) did not place these species near each other, Hellmayr (1935) placed T. rufigenis, $T$. cyanotis, and $T$. labradorides all near each other. Later classifications (e.g., Dickinson, 2003; Sibley and Monroe, 1990; Storer, 1970) included T. ruficervix with these species, which is not supported by the findings of our study.

Tangara phillipsi is one of the most recently described tanagers (Graves and Weske, 1987) and is only found on the slopes of Cerros del Sira, Peru. On the basis of plumage, Graves and Weske (1987) argued that the species was part of a monophyletic "black-capped" 
species complex that also included $T$. heinei, $T$. argyrofenges, and $T$. viridicollis. In addition, they suggested $T$. cyanoptera was closely related to the species in this group. Isler and Isler (1999) later included all of these in their species group 12. Graves and Weske (1987) argued that T. phillipsi was most closely related to T. heinei, and that T. phillipsi and T. heinei formed a superspecies. Subsequent classifications (e.g., Sibley and Monroe, 1990) have also treated $T$. phillipsi as specifically distinct and part of a species group with $T$. heinei. More recently, Vuilleumier et al. (1992) questioned the species status of $T$. phillipsi and argued that $T$. phillipsi was better treated as a subspecies of $T$. heinei. Ridgely and Tudor (2009) disagreed with Vuilleumier et al. (1992), citing the disjunct distribution of the two species and plumage differences. $T$. heinei is geographically isolated from T. phillipsi and occurs in the mountains of Venezuela, Colombia, and Ecuador. Prior to the current study, molecular phylogenetic analyses (Burns and Naoki, 2004; Sedano and Burns, 2010) included all these species except $T$. phillipsi and identified a strongly supported monophyletic clade consisting of $T$. heinei, $T$. argyrofenges, T. viridicollis, and T. cyanoptera. However, T. argyrofenges was only weakly differentiated genetically from $T$. heinei. The present study reports the first genetic data for T. phillipsi and shows that this species belongs in this clade as well, as anticipated by Graves and Weske (1987). However, we did not find strong support for the placement of $T$. heinei, $T$. argyrofenges, and $T$. phillipsi with respect to each other. All three are genetically very similar, although they each have unique plumage patterns and colors. Levels of uncorrected cyt $b$ sequence divergence among these species averages $0.34 \%$ (0.29-0.44\%), similar to that seen within species of other tanagers. However, levels of sequence variation can be affected by factors such as time since the cessation of gene flow, different rates of sequence evolution, population size, and past history of bottlenecks. Thus, if molecular data are to be used to assess species status, detailed phylogeographic studies involving numerous individuals across the range of a species are needed. Regardless of species status, our data indicate that these taxa have undergone rapid plumage evolution in the face of little genetic change.

In addition to T. rufigenis and T. phillipsi, the current study provides sequence data for three additional species of core tanagers that were not included in Sedano and Burns (2010): Gubernatrix cristata, Saltator rufiventris, and Paroaria baeri. G. cristata, the Yellow Cardinal, has had a complicated taxonomic history, having been classified at various times with cardinal-grosbeaks (e.g., Hellmayr, 1938; Ridgely and Tudor, 2009) or New World sparrows (e.g., Dickinson, 2003; Paynter and Storer, 1970; Rising, 2011; Sibley and Monroe, 1990; Webster and Webster, 1999). Tordoff's (1954) morphological study showed this species was not a cardinal; therefore, most recent classifications have placed Gubernatrix with the New World sparrows. Within this group, Gubernatrix is typically placed adjacent to the genus Paroaria and other Neotropical genera. Although Sibley and Monroe (1990) transferred many of these Neotropical sparrows to the tanagers, they kept Gubernatrix and Paroaria with the New World sparrows. Campagna et al. (2011) provided the first molecular phylogenetic analysis that included Gubernatrix. They analyzed 694 base pairs of COI and found that G. cristata was most closely related to Diuca diuca among the 39 species of tanagers included in their phylogeny. Although based on only a small fragment of DNA and only a relatively few number of species, this finding is confirmed by both Barker et al.'s (2013) genus-level sampling and the comprehensive species-level sampling of the current study. We found strong support (1.0 PP; 97\% bootstrap) for a sister relationship between G. cristata and D. diuca, which share a southern South American distribution and have hybridized in nature (Bertonatti and Guerra, 1997). Together, the clade containing $G$. cristata and $D$. diuca is most closely related to Neothraupis fasciata (1.0 PP; 88\% bootstrap), a species long considered a tanager (e.g., Hellmayr, 1935). Similarities between Neothraupis and Diuca are reflected in the taxonomic history of N. fasciata, which was previously classified in Diuca or Diucopsis (= 'Diuca-faced'). Although Diuca diuca and D. speculifera were once considered to be part of the same superspecies (Paynter and Storer, 1970), they are not closely related (see Diglossinae, Section 4.3.14 for $D$. speculifera). D. speculifera is the type species of Diuca; therefore, for $D$. diuca, we recommend using the available name Hedyglossa Reichenback 1851 (type $=D$. diuca) .

Our broad taxonomic sampling allowed us to identify cases, such as we found with Diuca, where species are only distantly related to their traditional congeners. Similarly, Saltator rufiventris belongs to Thraupinae in our phylogenies and is only distantly related to other members of Saltator (Saltatorinae, Section 4.3.8). Within the core tanager tree, we found strong support (1.0 PP; $100 \%$ bootstrap) for a clade containing S. rufiventris and the two species of Dubusia. This result agrees with earlier studies (Klicka et al., 2007; Barker et al., 2013) that included some of the data analyzed here. The close relationship of S. rufiventris and Dubusia agrees with similarities in habitats and elevational distribution of these three species. All species are Andean, with a center of abundance in the upper-montane elevational zone (Parker et al., 1996). Aspects of bill shape and plumage also make S. rufiventris a better fit with Dubusia than with the other saltators. Because the phylogenetic position of $S$. rufiventris renders Saltator paraphyletic, either Saltator rufiventris should be merged with Dubusia or a new genus name is needed for S. rufiventris.

We included an additional species of Paroaria (P. baeri) that was not included in Sedano and Burns (2010) and found strong support (1.0 PP; 93\% bootstrap) for this species being sister to the clade containing $P$. gularis and $P$. capitata. The close relationship of these taxa is consistent with their treatment as a superspecies by Sibley and Monroe (1990). In addition, other authors have considered $P$. baeri a subspecies of $P$. gularis, and $P$. baeri and $P$. gularis have hybridized (Lopes and Gonzaga, 2013). Dávalos and Porzecanski (2009) recently studied species limits in the genus Paroaria and included a molecular phylogenetic analysis of cyt $b$ sequences from multiple individuals of each species. For the species sampled in common, our phylogeny is largely congruent with that of Dávalos and Porzecanski (2009). Differences include the most basal split within Paroaria. Dávalos and Porzecanski (2009) found P. coronata to be the sister taxon to all other Paroaria, whereas we found a clade containing $P$. coronata and $P$. dominicana to be sister to the remaining species of Paroaria sampled. Although we found strong support for a sister relationship between $P$. dominicana and $P$. coronata, Dávalos and Porzecanski (2009) found weak support for a sister relationship between $P$. dominicana and $P$. nigrogenis, a species we did not sample. Differences between the trees of our study and those of Dávalos and Porzecanski (2009) are likely due to differences in sampling, with Dávalos and Porzecanski (2009) including more individuals and taxa and our study including more loci and more outgroups.

In addition to $P$. nigrogenis, we are only missing three other species of core tanagers: Tangara peruviana, T. cabanisi, and Thraupis glaucocolpa. Tangara peruviana is very similar in plumage to $T$. preciosa; thus it is likely the sister species of $T$. preciosa. $T$. cabanisi is likely closely related to T. palmeri based on similarities in plumage, size, habitat preference, and vocalizations (Hilty and Simon, 1977; Isler and Isler, 1999). Thraupis glaucocolpa was previously considered a subspecies of T. sayaca (e.g., Hellmayr, 1936; Storer, 1970); thus, these two species are likely sister taxa.

Sedano and Burns (2010) made several taxonomic recommendations for the core tanagers that are supported by the additional data presented here. Specifically, we recommend merging several species of Thraupis (Thraupis episcopus, T. sayaca, T. glaucocolpa, T. cyanoptera, T. palmarum, T. ornata, and T. abbas) with Tangara. 
This change is necessary to preserve the monophyly of Tangara, a well-known and widely used generic name. Because the name of Thraupis ornata will change to Tangara ornata, we agree with the recommendation of Sedano and Burns (2010) that the available junior synonym Tangara argentea Lafresnaye 1943 be used for the species currently known as Tangara ornata. The other species of Thraupis, $T$. cyanocephala, is not closely related to the other species in the genus; thus, a new generic name is necessary. No close relatives of $T$. cyanocephala were identified by our phylogenies; therefore, we recommend using the available generic name Sporathraupis Ridgway 1898 (type $=T$. cyanocephala) for this species. The two species of Buthraupis, B. montana and B. wetmorei, are not closely related to each other, and our phylogenies did not identify close relatives of either. Thus, we recommend retaining Buthraupis for the type species, B. montana, and using the available name Tephrophilus Moore 1934 (type $=B$. wetmorei) for B. wetmorei. The monophyly of Anisognathus was equivocal in Sedano and Burns (2010). In the present study, we recovered a monophyletic Anisognathus, but support for this clade was only moderate $(0.77 ; 58 \%$ bootstrap). Nonetheless, because Anisognathus is monophyletic, and because of morphological similarities of these species, we recommend retaining the current species composition of Anisognathus.

\subsection{Conclusion}

The phylogeny presented here has greatly strengthened our understanding of evolutionary relationships and patterns of diversification within Thraupidae. Moreover, this phylogeny sets the stage for studies of trait evolution in a comparative framework. Interspecific studies of various phenotypic traits in tanagers, such as bill morphology, vocal and plumage characters, and ecological niches, have the potential to reveal macroevolutionary patterns, such as correlated evolution and trait-dependent diversification. Using this phylogeny, future studies will shed light on the evolutionary processes that underlie speciation and the accumulation of phenotypic diversity in the largest radiation of Neotropical songbirds.

\section{Acknowledgments}

We thank the scientific collectors, collection managers, staff, and curators at the following institutions for providing the tissues used in this study: American Museum of Natural History; Academy of Natural Sciences, Philadelphia; University of Minnesota, Bell Museum of Natural History; Colección Ornitológica Phelps; Cornell University Museum of Vertebrates; Universidad del Valle, Colombia; Field Museum of Natural History; Instituto de Investigación de recursos Biológicos Alexander von Humboldt; Instituto de Ciencias Naturales, Universidad Nacional de Colombia; University of Kansas Natural History Museum; Natural History Museum of Los Angeles County; Louisiana State University Museum of Natural Science Collection of Genetic Resources; Museo Argentino de Ciencias Naturales "Bernardino Rivadavia"; University of Nevada Las Vegas, Barrick Museum of Natural History; Museum of Vertebrate Zoology, University of California, Berkeley; San Diego State University Museum of Biodiversity, Smithsonian Tropical Research Institute; University of Michigan Museum of Zoology; National Museum of Natural History (Smithsonian Institution); University of Washington, Burke Museum; and Zoological Museum, University of Copenhagen. We thank W. Mauck, R. Sedano, R. Keith, M. Alexander, and T. Shepherd for assistance with lab work. E. Dickinson and L. Christidis provided taxonomic advice. For advice on diversification analyses, we thank D. Rabosky. For suggestions on the manuscript, we thank C. Krajewski and two anonymous reviewers. Some of the photos in the graphical abstract were provided by M. Alexander and L. Calvert. This research was supported by the National Geographic Society (KJB) and the National Science Foundation (IBN-0217817 and DEB-0315416 to KJB; DEB-0315218 to IJL; DEB-0315469 to JK; and DEB-0316092 to SML and FKB). AJS was supported by a National Science Foundation Graduate Research Fellowship (2008074713).

\section{Appendix A. New subfamilies}

Subfamily Charitospizinae (new subfamily); Type genus: Charitospiza; Diagnosis: containing a single species, this subfamily is diagnosed by the species characters of Charitospiza eucosma Oberholser 1925; Genus: Charitospiza.

Subfamily Orchesticinae (new subfamily); Type genus: Orchesticus; Diagnosis: these two genera are united by a red iris and relatively thick and swollen bill with a hook on the upper mandible. Orchesticus has a dark eyeline, and the dark area around the eye in Parkerthraustes also includes the lores and ear coverts. We do not know any other morphological characters that unite these genera. Therefore, we also provide 31 unreversed molecular synapomorphies from the cyt $b$ gene. These include (numbered by their position in the gene alignment): C243G, T252C, C264T, C307A, C318T, A346G, C348A, A474C, C501A, A504C, C558A, G565C, C591T, T640C, C756A, C768T, C795T, A879C, C888T, C897T, A900C, C903A, A913C, C914T, C957T, C960T, C1074A, C1095T, T1115C, C1117T, and A1122G. Cladistically, we define this subfamily as the descendants of the common ancestor of Orchesticus abeillei and Parkerthraustes humeralis. Genera: Orchesticus and Parkerthraustes.

Subfamily Porphyrospizinae (new subfamily); Type genus: Porphyrospiza; Diagnosis: members of this subfamily are united by the presence of a bright yellow bill and dark lores in males. Additionally, we have identified 24 unreversed molecular synapomorphies across five genes: cyt $b$ : C615A and A729C; ND2: C32T, A39G, C81T, C300A, A484G, C549T, G574A, T715C, C736T, C843A, T924C, T951C, C1033A and T1035C; ACO1: C476T and A768G; MBI2: T190C; and RAG1: G426A, T622C, C1018T and A1870G. Cladistically, we define this subfamily as the descendants of the common ancestor of Incaspiza pulchra and Phrygilus alaudinus. Genera: Incaspiza, Phrygilus, and Porphyrospiza.

Subfamily Emberizoidinae (new subfamily); Type genus: Emberizoides; Diagnosis: Species of these three genera share a dark brown iris, green wing feathers, a yellow lower mandible, and a dark culmen. Additionally, we have identified 53 unreversed molecular synapomorphies across five genes: cyt $b$ : T161C and C174A; ND2: A39G, C63T, A118G, A277G, C278T, C292T, C366T, C396T, C460T, C477T, A484G, C549T, C606A, G640A, A703G, A722G, G724A, C747T, C762T, C793T, G826A, A844C, C850A, C902T, G948C, C969T, T992C, G993A and C1033A; ACO1: C56T, C74T, T290C, T550G and T825C; Fgbi5: G549A; Mbi2: G32A, G110A, T141A, T190C, G501T and T555C; and RAG1: T253C, T457C, T622C, C1018T, C1198T, T1603C, T1889C, A1984G, T2060C and T2347C. Cladistically, we define this subfamily as the descendants of the common ancestor of Coryphaspiza melanotis and Emberizoides herbicola. Genera: Coryphaspiza, Emberizoides, and Embernagra.

Subfamily Poospizinae (new description); Type genus: Poospiza; Diagnosis: We do not know any morphological characters that unite this diverse group. Instead, we have identified seven unreversed molecular synapomorphies across four genes: ND2: C1003A; ACO1: A768G; MBI2: G110A and T190C; and RAG1: T253C, C568G and A1984G. Cladistically, we define this subfamily as the descendants of the common ancestor of Xenospingus concolor and Poospiza melanoleuca. Genera: Cnemoscopus, Compsospiza, Cypsnagra, Donacospiza, Hemispingus, Nephelornis, Piezorina, Poospiza, Pyrrhocoma, Thlypopsis, Urothraupis, Xenospingus. 


\section{Appendix B. Supplementary material}

Supplementary data associated with this article can be found, in the online version, at http://dx.doi.org/10.1016/j.ympev.2014. 02.006 .

\section{References}

Akaike, H., 1973. Information theory and an extension of the maximum likelihood principle. In: Petrov, B.N., Csaki, F. (Eds.), Second International Symposium on Information Theory. Akademiai Kiado, Budapest, pp. 267-281.

Alfaro, M.E., Santini, F., Brock, C., Alamillo, H., Dornburg, A., Rabosky, D.L., Carnevale, G., Harmon, L.J., 2009. Nine exceptional radiations plus high turnover explain species diversity in jawed vertebrates. Proc. Natl. Acad. Sci. USA 106, 13410 13414.

Allen, J.A., 1891. On a collection of birds from Chapada, Matto Grosso, Brazil, made by Mr. Herbert H. Smith. Part I-Oscines. Bull. Am. Mus. Nat. Hist. 3, 337-380.

American Ornithologists' Union, 1983. Check-List of North American Birds, sixth ed. American Ornithologists' Union, Washington, DC.

American Ornithologists' Union, 1998. Check-List of North American Birds, seventh ed. American Ornithologists' Union, Washington, DC.

Areta, J.I., Pearman, M., Ábalos, R., 2012. Taxonomy and biogeography of the Monte Yellow-Finch (Sicalis mendozae): understanding the endemic avifauna of Argentina's Monte Desert. Condor 114, 654-671.

Assis, C.P., Raposo, M.A., Parrini, R., 2007. Validação de Poospiza cabanisi Bonaparte, 1850 (Passeriformes: Emberizidae). Rev. Bras. Ornitol. 15, 103-112.

Barker, F.K., Burns, K.J., Klicka, J., Lanyon, S.M., Lovette, I.J., 2013. Going to extremes: contrasting rates of diversification in a recent radiation of New World passerine birds. Syst. Biol. 62, 298-320.

Barker, F.K., Cibois, A., Schikler, P., Feinstein, J., Cracraft, J., 2004. Phylogeny and diversification of the largest avian radiation. Proc. Natl. Acad. Sci. USA 101, $11040-11045$.

Barreira, A.S., Garcia, G., Lijtmaer, D.A., Lougheed, S.C., Tubaro, P.L., 2008. Blue males and green females: sexual dichromatism in the Blue Dacnis (Dacnis cayana) and the Swallow Tanager (Tersina viridis). Ornitol. Neotrop. 19, 441-450.

Bates, J.M., Parker III, T.A., Capparella, A.P., Davis, T.J., 1992. Observations on the campo, cerrado and forest avifaunas of eastern Dpto. Santa Cruz, Bolivia, including 21 species new to the country. Bull. Brit. Ornithol. Club 112, 86-98.

Bauer, C., Pacheco, J.F., Venturini, A.C., Whitney, B.M., 2000. Rediscovery of the Cherry-throated Tanager Nemosia rourei in southern Espirito Santo, Brazil. Bird Conserv. Int. 10, 97-108.

Beauchamp, G., Goodale, E., 2011. Plumage mimicry in avian mixed-species flocks: more or less than meets the eye? The Auk 128, 487-496.

Beecher, W.J., 1951. Convergence in the Coerebidae. Wilson Bull. 63, 274-287.

Bellemain, E., Bermingham, E., Ricklefs, R., 2008. The dynamic evolutionary history of the bananaquit (Coereba flaveola) in the Caribbean revealed by a multigene analysis. BMC Evol. Biol. 8, 240

Bertonatti, C., Guerra, A. López, 1997. Hibridación entre Cardenal Amarillo (Gubernatrix cristata) y Diuca Común (Diuca diuca) en estado silvestre, en Argentina. El Hornero 14, 243-246.

BirdLife International, 2013a. Species Factsheet: Charitospiza eucosma. <http:// www.birdlife.org> (accessed 30.10.13).

BirdLife International, 2013b. Species Factsheet: Conirostrum tamarugense. <http:// www.birdlife.org $>$ (accessed 12.09.13).

Bleiweiss, R., 2008. Phenotypic integration expressed by carotenoid-bearing plumages of tanager-finches (Thraupini, Emberizinae) across the avian visible spectrum. Biol. J. Linn. Soc. 93, 89-109.

Bledsoe, A.H., 1988. Nuclear DNA evolution and phylogeny of the New World nineprimaried oscines. The Auk 105, 504-515.

Bond, J., 1951. Taxonomic notes on South American birds. The Auk 68, 527-529.

Brewer, D., 2011. Black-throated Saltator Saltator atricollis. In: del Hoyo, J., Elliot, A., Christie, D. (Eds.), Handbook of the Birds of the World. Tanagers to New World Blackbirds, vol. 16. Lynx Edicions, Barcelona, p. 419.

Burns, K.J., 1997. Molecular systematics of tanagers (Thraupinae): evolution and biogeography of a diverse radiation of Neotropical birds. Mol. Phylogenet. Evol 8, 334-348.

Burns, K.J., Hackett, S.J., Klein, N.K., 2002. Phylogenetic relationships and morphological diversity in Darwin's finches and their relatives. Evolution 56, 1240-1252.

Burns, K.J., Hackett, S.J., Klein, N.K., 2003. Phylogenetic relationships of Neotropical honeycreepers and the evolution of feeding morphology. J. Avian Biol. 34, 360370.

Burns, K.J., Naoki, K., 2004. Molecular phylogenetics and biogeography of neotropical tanagers in the genus Tangara. Mol. Phylogenet. Evol. 32, 838-854.

Burns, K.J., Racicot, R.A., 2009. Molecular phylogenetics of a clade of lowland tanagers: implications for avian participation in the Great American Interchange. The Auk 126, 635-648.

Burns, K.J., Shultz, A.J., 2012. Widespread cryptic dichromatism and ultraviolet reflectance in the largest radiation of Neotropical songbirds: implications of accounting for avian vision in the study of plumage evolution. The Auk 129 211-221.

Burnham, K.P., Anderson, D.R., 2002. Model Selection and Multimodel Inference. Springer, New York.
Buzzetti, D., Carlos, B.A., 2005. A redescoberta do tiê-bicudo (Conothraupis mesoleuca) (Berlioz, 1939). Atual. Ornito. 127, 16-17.

Campagna, L., Geale, K., Handford, P., Lijtmaer, D.A., Tubaro, P.L., Lougheed, S.C., 2011. A molecular phylogeny of the Sierra-Finches (Phrygilus, Passeriformes): extreme polyphyly in a group of Andean specialists. Mol. Phylogenet. Evol. 61, 521-533.

Campagna, L., Benites, P., Lougheed, S.C., Lijtmaer, D.A., Di Giacomo, A.S., Eaton, M.D., Tubaro, P.L., 2012. Rapid phenotypic evolution during incipient speciation in a continental avian radiation. Proc. Roy. Soc. B 279, 1847-1856.

Candia-Gallardo, C.E., Silveira, L.F.B., Kuniy, A.A., 2010. A new population of the Cone-billed Tanager Conothraupis mesoleuca, with information on the biology, behaviour and type locality of the species. Bird Conserv. Int. 20, 149-160.

Cavalcanti, R.B., Alves, M.A.S., 1997. Effects of fire on savanna birds in Central Brazil. Ornitol. Neotrop. 8, 85-87.

Chapman, F.M., 1926. The distribution of bird-life in Ecuador. Bull. Am. Mus. Nat. Hist. 55, 1-784.

Clark, G.A., 1986. Systematic interpretations of foot-scute patterns in Neotropical finches. Wilson Bull. 98, 594-597.

Chaves, J.A., Hidalgo, J.R., Klicka, J., 2013. Biogeography and evolutionary history of the Neotropical genus Saltator (Aves: Thraupini). J. Biogeogr.. http://dx.doi.org/ $10.1111 /$ jbi.12150.

Clements, J.F., Schulenberg, T.S., Iliff, M.J., Sullivan, B.L., Wood, C.L., Roberson, D. 2013. The Clements Checklist of Birds of the World: Version 6.8. <http:/| www.birds.cornell.edu/clementschecklist/download/> (accessed 04.10.13).

Dávalos, L.M., Porzecanski, A.L., 2009. Accounting for molecular stochasticity in systematic revisions: species limits and phylogeny of Paroaria. Mol. Phylogenet. Evol. 53, 234-248.

Demastes, J.W., Remsen, J.V., 1994. The genus Caryothraustes (Cardinalinae) is not monophyletic. Wilson Bull. 106, 733-738.

Dickerman, R.W., 1986. Notes on the plumages of the Paramo Seedeater (Catamenia homochroa). The Auk 103, 227-230.

Dickinson, E.C., 2003. The Howard and Moore Complete Checklist of the Birds of the World. Princeton University Press, Princeton.

Diniz, P., Ramos, D.M., Chemin, N., 2013. Breeding biology of Coal-crested Finches. Wilson J. Ornithol. 125, 592-599.

Drummond, A.J., Ho, S.Y., Phillips, M.J., Rambaut, A., 2006. Relaxed phylogenetics and dating with confidence. PLoS Biol. 4, e88.

Drummond, A.J., Suchard, M.A., Xie, D., Rambaut, A., 2012. Bayesian phylogenetics with BEAUti and the BEAST 1.7. Mol. Biol. Evol. 29, 1969-1973.

Eisenmann, E., Short, L.L., 1982. Systematics of the avian genus Emberizoides (Emberizidae). Am. Mus. Novit. 2740.

Etienne, R.S., Haegeman, B., Stadler, T., Aze, T., Pearson, P.N., Purvis, A., Phillimore, A.B., 2012. Diversity-dependence brings molecular phylogenies closer to agreement with the fossil record. Proc. Roy. Soc. B 279, 1300-1309.

Fjeldså, J., 1992. Biogeographic patterns and evolution of the avifauna of relict highaltitude woodlands of the Andes. Steenstrupia 18, 9-62.

Fjeldså, J., Krabbe, N., 1990. Birds of the High Andes. Zoological Museum, Univ. Copenhagen, Copenhagen.

Fjeldså, J., Rahbek, C., 2006. Diversification of tanagers, a species rich bird group, from lowlands to montane regions of South America. Integr. Comp. Biol. 46, 72 81.

Freeman, B.G., Arango, J.A., 2010. The nest of the Gold-ringed Tanager (Bangsia aureocincta), a Colombian endemic. Ornito. Colombiana 9, 71-75.

George, W.G., 1964. Rarely seen songbirds of Peru's high Andes. Nat. Hist. 73, 26-29.

Grant, P.R., 1999. Ecology and Evolution of Darwin's Finches. Princeton University Press, Princeton, New Jersey.

Grant, P.R., Grant, B.R., 2008. How and Why Species Multiply: The Radiation of Darwin's Finches. Princeton University Press, Princeton, New Jersey.

Graves, G.R., Weske, J.S., 1987. Tangara phillipsi, a new species of tanager from the Cerros del Sira, eastern Peru. Wilson Bull. 99, 1-6.

Gray, G.R., 1870. Hand-List of Genera and Species of Birds. Taylor and Francis, London.

Gwynne, J.A., Ridgely, R.S., Tudor, G., Argel, M., 2010. Wildlife Conservation Society Birds of Brazil: The Pantanal and Cerrado of Central Brazil. Comstock Pub. Associates, London.

Hackett, S.J., 1996. Molecular phylogenetics and biogeography of tanagers in the genus Ramphocelus (Aves). Mol. Phylogenet. Evol. 5, 368-382.

Hellack, J.J., Schnell, G.D., 1977. Phenetic analysis of the subfamily Cardinalinae using external and skeletal characters. Wilson Bull. 89, 130-148.

Hellmayr, C.E., 1932. The birds of Chile. Field Mus. Nat. Hist., Zool. Ser. 19, 1-472.

Hellmayr, C.E., 1935. Catalogue of birds of the Americas and the adjacent islands. Field Mus. Nat. Hist., Zool. Ser. 13 (Pt. 9).

Hellmayr, C.E., 1936. Catalogue of birds of the Americas and the adjacent islands. Field Mus. Nat. Hist. Publ. Zool. Ser. 13 (Pt. 9).

Hellmayr, C.E., 1938. Catalogue of birds of the Americas and the adjacent islands. Field Mus. Nat. Hist. Publ. Zool. Ser. 13 (Pt. 11).

Hilty, S.L., Simon, D., 1977. The Azure-rumped Tanager in Mexico with comparative remarks on the Gray-and-gold Tanager. The Auk 94, 605-606.

Hilty, S.L., Parker III, T.A., Silliman, J., 1979. Observations on Plush-capped Finches in the Andes with a description of the juvenal and immature plumages. Wilson Bull. 91, 145-148.

Hilty, S.L., 2011. Family Thraupidae (Tanagers). In: del Hoyo, J., Elliott, A., Christie, D.A. (Eds.), Handbook of the Birds of the World. Tanagers to New World Blackbirds, vol. 16. Lynx Edicions, Barcelona, pp. 46-329.

Howard, R., Moore, A., 1991. A Complete Checklist of the Birds of the World. Academic Press, London. 
Ingels, J., 2007. Additional information on the breeding biology of the Black-andwhite Tanager (Conothraupis speculigera) in Ecuador. Boletín SAO 17, 98-103.

Isler, M.L., Isler, P.R., 1999. The Tanagers. Smithsonian Institution Press, Washington, DC.

Jaramillo, A. 2011a. Coal-crested Finch Charitospiza eucosma. In: del Hoyo, J., Elliott, A., Christie, D.A. (Eds.), Handbook of the Birds of the World. Tanagers to New World Blackbirds, vol. 16. Lynx Edicions, Barcelona, p. 639.

Jaramillo, A., 2011b. Many-colored Chaco Finch Saltatricula multicolor. In: del Hoyo, J., Elliott, A., Christie, D.A. (Eds.), Handbook of the Birds of the World. Tanagers to New World Blackbirds, vol. 16. Lynx Edicions, Barcelona, p. 683.

Jaramillo, A., 2011c. Black-masked Finch Coryphaspiza melanotis. In: del Hoyo, J., Elliott, A., Christie, D.A. (Eds.), Handbook of the Birds of the World. Tanagers to New World Blackbirds, vol. 16. Lynx Edicions, Barcelona, p. 640.

Jaramillo, A., 2011d. Patagonian Sierra-finch Phrygilus patagonicus. In: del Hoyo, J., Elliott, A., Christie, D.A. (Eds.), Handbook of the Birds of the World. Tanagers to New World Blackbirds, vol. 16. Lynx Edicions, Barcelona, pp. 606-607.

Jaramillo, A., 2011e. White-winged Diuca-finch Diuca speculifera. In: del Hoyo, J., Elliott, A., Christie, D.A. (Eds.), Handbook of the Birds of the World. Tanagers to New World Blackbirds, vol. 16. Lynx Edicions, Barcelona, p. 616.

Jaramillo, A., 2011f. Ash-breasted Sierra-finch Phrygilus plebejus. In: del Hoyo, J., Elliott, A., Christie, D.A. (Eds.), Handbook of the Birds of the World. Tanagers to New World Blackbirds, vol. 16. Lynx Edicions, Barcelona, p. 608.

Jaramillo, A., 2011g. Plumbeous Sierra-finch Phrygilus unicolor. In: del Hoyo, J., Elliott, A., Christie, D.A. (Eds.), Handbook of the Birds of the World. Tanagers to New World Blackbirds, vol. 16. Lynx Edicions, Barcelona, p. 608.

Jetz, W., Thomas, G.H., Joy, J.B., Hartmann, K., Mooers, A.O., 2012. The global diversity of birds in space and time. Nature 491, 444-448.

Johnson, A.W., Millie, W.R., 1972. A new species of conebill (Conirostrum) from northern Chile. In: Johnson, A.W. (Ed.), Supplement to the Birds of Chile and Adjacent Regions of Argentina, Bolivia, and Peru. Platt Establecimientos Gráficos S.A., Buenos Aires, pp. 3-8

Kendall, D.G., 1948. On the generalized birth-and-death process. Ann. Math. Stat. 19, 1-15.

Klicka, J., Burns, K.J., Spellman, G.M., 2007. Defining a monophyletic Cardinalini: a molecular perspective. Mol. Phylogenet. Evol. 45, 1014-1032.

Lack, D., 1947. Darwin's Finches. Cambridge Univ. Press, London.

Larkin, M.A., 2007. ClustalW and ClustalX version 2.0. Bioinformatics 23, $2947-$ 2948.

Lebbin, D., 2005. Aberrant plumage in a Black-and-white Tanager (Conothraupis speculigera). Boletín SAO 15, 100-104.

Lebbin, D., 2006. Correction of "aberrant plumage" in a Black-and-white Tanager (Conothraupis speculigera). Boletín SAO 16, 100-101.

Lloyd, H., Walker, B., Aucca Chutas, C., Schmitt, F., 2005. Range extension of Shorttailed Finch Idiopsar brachyurus in Perú with notes on its behaviour, plumage and soft-part colouration and nomenclature. Cotinga 23, 48-51.

Lloyd, H., 2009. In: Schulenberg, T.S. (Ed.) Short-tailed Finch (Idiopsar brachyurus) Neotropical Birds Online. Cornell Lab of Ornithology, Ithaca, New York (USA). <http://neotropical.birds.cornell.edu/portal/species/overview?p_p_spp=42732> (accessed 4.10.13)

Lopes, L.E., Gonzaga, L.P., 2013. Taxonomy, natural history, and conservation of Paroaria baeri (Aves: Thraupidae). Trop. Zool. 26, 87-103.

Lougheed, S.C., Freeland, J.R., Hanford, P., Boag, P.T., 2000. A molecular phylogeny of Warbling-Finches (Poospiza) paraphyly in a Neotropical Emberizid genus. Mol. Phylogenet. Evol. 17, 367-378.

Lovette, I.J., Bermingham, E., 2002. What is a Wood-Warbler? Molecular characterization of a monophyletic Parulidae. The Auk 119, 695-714.

Lowe, P.R., 1923. Notes on some land birds of the Tristan da Cunha group collected by the 'Quest' expedition. Ibis 65, 511-528.

Lowery, G.H., Monroe, B.L., 1968. Family Parulidae. In: Paynter, R.A., Jr. (Ed.), Peter's Check-List of Birds of the World, vol. 14. Museum of Comparative Zoology, Cambridge, Massachusetts, pp. 3-93.

Lucas, F.A., 1895. Osteological and pterylographical characters of the Procniatidae. Proc. US Nat. Mus. 18, 505-507.

Mason, N.A., Burns, K.J. 2010. In: Schulenberg, T.S. (Ed.), Giant Conebill (Oreomanes fraseri), Neotropical Birds Online. Cornell Lab of Ornithology, Ithaca, New York (USA). <http://neotropical.birds.cornell.edu/portal/species/overview?p_p_spp= 589196> (accessed 30.10.13).

Mason, N.A., Burns, K.J., 2013. Molecular phylogenetics of the Neotropical seedeaters and seed-finches (Sporophila, Oryzoborus, Dolospingus). Ornitol. Neotrop. 24, 139-155.

Marín, M., Kiff, L.F., Peña, L., 1989. Notes on Chilean birds, with descriptions of two new subspecies. Bull. Brit. Ornithol. Club 109, 66-82.

Mauck, W.M., Burns, K.J., 2009. Phylogeny, biogeography, and recurrent evolution of divergent bill types in the nectar-stealing flowerpiercers (Thraupini: Diglossa and Diglossopis). Biol. J. Linn. Soc. 98, 14-28.

Mallarino, R., Campàs, O., Fritz, J.A., Burns, K.J., Weeks, O.G., Brenner, M.P., Abzhanov, A., 2012. Closely related bird species demonstrate flexibility between beak morphology and underlying developmental programs. Proc. Natl. Acad. Sci. USA 109, 16222-16227.

Mayr, E., Vuilleumier, F., 1983. New species of birds described from 1966 to 1975. J. Ornithol. 124, 217-232.

Meyer De Schauensee, R., 1966. The Species of Birds of South America and Their Distribution. Livingston Publishing Co., Narberth, Pennsylvania.

Meyer De Schauensee, R., 1970. A Guide to the Birds of South America. Livingston Publishing Co., Wynnewood, Pennsylvania.
Miller, M.A., Pfeiffer, W., Schwartz, T., 2010. Creating the CIPRES Science Gateway for inference of large phylogenetic trees. In: Proceedings of the Gateway Computing Environments Workshop (GCE), 14 November, 2010, New Orleans, LA, pp. 1-8.

Miller, W.De.W., 1928. Schistospiza Sharpe not separable from Lophospingus Cabanis. The Auk 45, 380-381.

Moynihan, M., 1962. Display patterns of tropical American "nine-primaried" songbirds II. Some species of Ramphocelus. The Auk 79, 655-686.

Moynihan, M., 1966. Display patterns of tropical American "nine-primaried" songbirds. IV. The Yellow-rumped Tanager. Smith. Misc. Coll. 149 (5).

Morony Jr, J.J., 1985. Systematic relations of Sericossypha albocristata (Thraupinae). Ornithol. Monogr. 36, 382-389.

Nee, S., May, R.M., Harvey, P.H., 1994. The reconstructed evolutionary process. Phil. Trans. Roy. Soc. B 344, 305-311.

Nee, S., Mooers, A., Harvey, P.H., 1992. Tempo and mode of evolution revealed from molecular phylogenies. Proc. Natl. Acad. Sci. USA 89, 8322-8326.

Nylander, J.A.A., Wilgenbusch, J.C., Warren, D.L., Swofford, D.L., 2008. AWTY (are we there yet?): a system for graphical exploration of MCMC convergence in Bayesian phylogenetics. Bioinformatics 24, 58-583.

Oberholser, H.C., 1905. Notes on the nomenclature of certain genera of birds. Smith. Misc. Coll. 48, 59-68.

Olson, S.L., 1986. Catamblyrhynchus and Paradoxornis: an unremarked instance of convergence in bill morphology for feeding on bamboo. Bull. Brit. Ornithol. Club 106, 161-163.

Orenstein, R.I., 2011. Family Cardinalidae (Cardinals). In: del Hoyo, J., Elliott, A. Christie, D.A. (Eds.), Handbook of the Birds of the World. Tanagers to New World Blackbirds. Lynx Edicions, Barcelona, pp. 330-427.

Parker III, T.A., Stotz, D.F., Fitzpatrick, J.W., 1996. Ecological and distributional databases. In: Stotz, D.F., Fitzpatrick, J.W., Parker, T.A., III, Moskovits, D.K. (Eds.), Neotropical Birds: Ecology and Conservation. Univ. of Chicago Press, Chicago, Illinois, pp. 113-436.

Paynter, R.A., Storer, R.W., 1970. Subfamilies Emberizinae, Catamblyrhynchinae, Cardinalinae, Thraupinae, and Tersininae. In: Paynter Jr., R.A. (Ed.), Check-List of Birds of the World, vol. 13. Museum of Comparative Zoology, Cambridge, MA.

Petren, K., Grant, P.R., Grant, B.R., Keller, L.F., 2005. Comparative landscape genetics and the adaptive radiation of Darwin's finches: the role of peripheral isolation. Mol. Ecol. 14, 2943-2957.

Philippi, B.R.A., 1942. Notas sobre Aves Chilenas. Bol. Mus. Nac. Hist. Nat. Chile 20, 81-89.

Phillimore, A.B., Price, T.D., 2008. Density-dependent cladogenesis in birds. PLoS Biol. 6, 0483-0489.

Posada, D., 2008. JModelTest: phylogenetic model averaging. Mol. Biol. Evol. 25 1253.

Price, T.D., 2011. Adaptive radiations: there's something about finches. Curr. Biol 21, R953-R955.

Pybus, O.G., Harvey, P.H., 2000. Testing macro-evolutionary models using incomplete molecular phylogenies. Proc. Roy. Soc. B 267, 2267-2272.

Rabosky, D.L., 2006. LASER: a maximum likelihood toolkit for detecting temporal shifts in diversification rates from molecular phylogenies. Evol. Bioinform. 2 247-250.

Rabosky, D.L., 2010. Primary controls on species richness in higher taxa. Syst. Biol 59, 634-645.

Rabosky, D.L., Santini, F., Eastman, J., Smith, S.A., Sidlauskas, B., Chang, J., Alfaro M.E., 2013. Rates of speciation and morphological evolution are correlated across the largest vertebrate radiation. Nat. Commun. 4, 1-8.

Rabosky, D.L., 2014. Automatic detection of key innovations, rate shifts, and diversity-dependence on phylogenetic trees. PlosONE 9:e89543.

Rabosky, D.L., Matute, D.R., 2013. Macroevolutionary speciation rates are decoupled from the evolution of intrinsic reproductive isolation in Drosophila and birds. Proc. Natl. Acad. Sci. USA 110, 15354-15359.

Rambaut, A., Drummond, A.J., 2007. Tracer v1.4. <http://beast.bio.ed.ac.uk/Tracer> (accessed 04.10.13).

Remsen Jr, J.V., 1997. A new genus for the Yellow-shouldered Grosbeak. Ornithol Monogr. 48, 89-90.

Remsen Jr, J.V., Cadena, C.D., Jaramillo, A., Nores, M., Pacheco, J.F., Pérez-Emán, J. Robbins, M.B., Stiles, F.G., Stotz, D.F., Zimmer, K.J., 2013. A Classification of the Bird Species of South America. American Ornithologists' Union. <http:/| www.museum.lsu.edu/ Remsen/SACCBaseline.html> (accessed 17.09.13).

Restall, R., Rodner, C., Lentino, M., 2006. Birds of Northern South America. Yale University Press, New Haven, Connecticut.

Ridgely, R.S., Tudor, G., 1989. The Birds of South America, The Oscine Passerines, vol 1. Univ. of Texas Press, Austin.

Ridgely, R.S., Tudor, G., 2009. Field Guide to the Songbirds of South America: The Passerines. Univ. of Texas Press, Austin.

Ridgely, R.S., Greenfield, P.J., 2001. The Birds of Ecuador. Cornell Univ. Press, Ithaca,

Ridgway, R., 1901a. The birds of North and Middle America, part I. Bull. US Natl. Mus. 50.

Ridgway, R., 1901b. New birds of the families Tanagridae and Icteridae. Proc. Wash. Acad. Sci. 3, 149-155.

Ridgway, R., 1902. The birds of North and Middle America, part II. Bull. US Natl. Mus. 50.

Rising, J.D., 2011. Family Emberizidae (Buntings and New World Sparrows). In: del Hoyo, J., Elliott, A., Christie, D.A. (Eds.), Handbook of the Birds of the World Tanagers to New World Blackbirds. Lynx Edicions, Barcelona, pp. 428-683.

Ryan, P.G., 2007. Field Guide to the Animals and Plants of Tristan da Cunha and Gough Island. Pisces Publications, Newbury. 
Ryan, P.G., Bloomer, P., Moloney, C.L., Grant, T.J., Delport, W., 2007. Ecological speciation in South Atlantic Finches. Science 315, 1420-1423.

Ryan, P.G., Klicka, L.B., Barker, F.K., Burns, K.J., 2013. The origin of finches on Tristan da Cunha and Gough Island, central South Atlantic Ocean. Mol. Phylogenet. Evol. 69, 299-305.

Sato, A., O'hUigin, C., Figueroa, F., Grant, P.R., Grant, B.R., Tichy, H., Klein, J., 1999. Phylogeny of Darwin's finches as revealed by mtDNA sequences. Proc. Natl. Acad. Sci. USA 96, 5101-5106.

Sato, A., Tichy, H., O’hUigin, C., Grant, P.R., Grant, B.R., Klein, J., 2001. On the origin of Darwin's finches. Mol. Biol. Evol. 18, 299-311.

Sazima, I., 2010. Five instances of bird mimicry suggested for Neotropical birds: a brief reappraisal. Rev. Bras. Ornitol. 18, 328-335.

Schaefer, E., 1953. Contribution to the life history of the Swallow-Tanager. The Auk $70,403-460$

Schulenberg, T.S., 1985. An intergeneric hybrid conebill (Conirostrum x Oreomanes) from Peru. Ornithol. Monogr. 36, 390-395.

Sclater, P.L., 1854. On two new species of Dacnis, and on the general arrangement on the genus. Proc. Zool. Soc. Lond. 22, 251-252.

Sclater, P.L., 1856. On a new tanager of the genus Calliste. Proc. Zool. Soc. Lond. 24, 311.

Sclater, P.L., 1860. List of birds collected by Mr. Fraser in the vicinity of Quito, and during excursions to Pichincha and Chimborazo; with notes and descriptions of new species. Proc. Zool. Soc. Lond. 28, 73-83.

Sclater, P.L., 1886. Catalogue of the Birds in the British Museum, vol. XI. British Museum, London.

Scott, D.A., 1997. A possible re-sighting of the Cherry-throated Tanager Nemosia rourei in Espirito Santo, Brazil. Cotinga 7, 61-63.

Sedano, R.E., Burns, K.J., 2010. Are the Northern Andes a species pump for Neotropical birds? Phylogenetics and biogeography of a clade of Neotropical tanagers (Aves: Thraupini). J. Biogeogr. 37, 325-343.

Seutin, G., Brawn, J., Ricklefs, R.E., Bermingham, E., 1993. Genetic divergence among populations of a tropical passerine, the streaked saltator (Saltator albicollis). The Auk 110, 117-126.

Sharpe, R.B., 1888. Catalogue of the Birds of the British Museum, vol. XII. British Museum, London.

Short, L.L., 1975. A zoogeographic analysis of the South American chaco avifauna. Bull. Am. Mus. Nat. Hist. 154, 163-352.

Shultz, A.J., Burns, K.J., 2013. Plumage evolution in relation to light environment in a novel clade of Neotropical tanagers. Mol. Phylogenet. Evol. 66, 112-125.

Sick, H., 1993. Birds in Brazil: A Natural History. Princeton University Press, Princeton.

Sibley, C.G., Ahlquist, J.E., 1990. Phylogeny and Classification of Birds. Yale Univ. Press, New Haven.

Sibley, C.G., Monroe, B.L., 1990. Distribution and Taxonomy of the Birds of the World. Yale University Press, New Haven, Connecticut.

Skutch, A.F., 1954. Life histories of Central American birds. In: Pacific Coast Avifauna. Cooper Ornithological Society, Berkeley, California, USA.

Stamatakis, A., 2006. RAxML-VI-HPC: maximum likelihood-based phylogenetic analyses with thousands of taxa and mixed models. Bioinformatics 22, 26882690.

Stamatakis, A., Hoover, P., Rougemont, J., 2008. A rapid bootstrap algorithm for the RAxML web servers. Syst. Biol. 57, 758-771.

Steadman, D., 1982. The origin of Darwin's finches (Fringillidae, Passeriformes) Trans. San Diego Soc. Nat. Hist. 19, 279-296.

Storer, R.W., 1960. Notes on the systematics of the tanager genus Conothraupis. The Auk 77, 350-351.

Storer, R.W., 1969. What is a tanager? Living Bird 8, 127-136.

Storer, R.W., 1970. Subfamilies Thraupinae and Tersininae. In: Paynter, R.A., Jr. (Ed.), Check-List of Birds of the World, vol. 13. Museum of Comparative Zoology, Cambridge, MA, pp. 246-409.
Sugiura, N., 1978. Further analysis of the data by Akaike's information criterion and the finite corrections. Commun. Stat. Theory Methods A7, 13-26.

Sushkin, P.P., 1924. On the Fringillidae and allied groups. Bull. Brit. Ornithol. Club $45,36-39$.

Tonnis, B., Grant, P.R., Grant, B.R., Petren, K., 2005. Habitat selection and ecological speciation in Galápagos warbler finches (Certhidea olivacea and Certhidea fusca). Proc. Roy. Soc. B 272, 819-826.

Tordoff, H.B., 1954. A systematic study of the avian family Fringillidae based on the structure of the skull. Misc. Publ. Mus. of Zool. Univ. Michigan 81.

Venturini, A.C., de Paz, P.R., Kirwan, G.M., 2005. A new locality and records of Cherry-throated Tanager Nemosia rourei in Espírito Santo, south-east Brazil, with fresh natural history data for the species. Cotinga 24, 60-70.

Vogt, C.A., 2006. Secondary nectar robbing, a previously unsubstantiated foraging behavior of the Cinereous Conebill (Conirostrum cinereum). Ornitol. Neotrop. 17, 613-617.

Vuilleumier, F., 1984. Patchy distribution and systematics of Oreomanes fraseri (Aves: Coerebidae) of Andean Polylepis woodlands. Am. Mus. Novit. 2777, 1-17.

Vuilleumier, F., 1967. Speciation in High Andean Birds. Ph.D. Dissertation, Harvard University, $444 \mathrm{pp}$.

Vuilleumier, F., 1991. A quantitative survey of speciation phenomena in Patagonian birds. Ornitol. Neotrop. 2, 5-28.

Vuilleumier, F., LeCroy, M., Mayr, E., 1992. New species of birds described from 1981 to 1990. Bull. Brit. Orn. Club 122, 267-309.

Webster, J.D., 1988. Skeletons and the genera of tanagers. Proc. Indiana Acad. Sci. 98, 581-593.

Webster, J.D., Webster, J.R., 1999. Skeletons and the genera of sparrows (Emberizinae). The Auk 115, 1054-1074.

Weir, J.T., Bermingham, E., Schluter, D., 2009. The great American biotic interchange in birds. Proc. Natl. Acad. Sci. 106, 21737-21742.

Weir, J.T., Schluter, D., 2008. Calibrating the avian molecular clock. Mol. Ecol. 17, 231-2328.

Wester, P., Claßen-Bockhoff, R., 2006. Hummingbird pollination in Salvia haenkei (Lamiaceae) lacking the typical lever mechanism. Plant Syst. Evol. 257, 133146.

Wetmore, A., 1960. A classification for the birds of the world. Smith. Misc. Coll. 139.

Willis, E.O., 1976. Similarity of a tanager (Orchesticus abeillei) and an Ovenbird (Philydor rufus): a possible case of mimicry. Ciência e Cultura 28, 1492-1493.

Willis, E.O., 1985. Behavior and systematic status of Gray-headed Tanagers (Trichothraupis penicillata, Emberizidae). Naturalia 10, 113-145.

Willis, E.O., 1989. Mimicry in bird flocks of cloud forests in south-eastern Brazil. Rev. Bras. Biol. 49, 615-619.

Witt, C.C., 2005. Syntopic breeding suggests mimicry of the Black-and-white Seedeater (Sporophila luctuosa) by the Black-and-white Tanager (Conothraupis speculigera). Ornitol. Neotrop. 16, 387-396.

Wolters, H.E., 1975-1982. Die vogelarten der erde, Paul Parey, Hamburg and Berlin.

Yule, G.U., 1924. A mathematical theory of evolution, based on the conclusions of Dr. J. C. Willis, F.R.S. Phil. Trans. Roy. Soc B 213, 21-87.

Yuri, T., Mindell, D.P., 2002. Molecular phylogenetic analysis of Fringillidae, "New World nine-primaried oscines" (Aves: Passeriformes). Mol. Phylogenet. Evol. 23, 229-243.

Zimmer, J.T., 1942. Studies of Peruvian birds, No. 43. Notes on the genera Dacnis, Xenodacnis, Coereba, Conirostrum, and Oreomanes. Am. Mus. Novit. 1193, 1-16.

Zimmer, J.T., 1947. Studies of Peruvian birds, No. 52. The genera Sericossypha, Chlorospingus, Cnemoscopus, Hemispingus, Conothraupis, Chlorornis, Lamprospiza, Cissopis, and Schistochlamys. Am. Mus. Novit. 1367, 1-26.

Zimmer, J.T., 1952. A new finch from northern Peru. J. Wash. Acad. Sci. 42, 103-104.

Zink, R.M., 2002. A new perspective on the evolutionary history of Darwin's finches. The Auk 119, 864-871. 